In cooperation with the Houston-Galveston Area Council

Hydrologic, Water-Quality, and Sediment-Quality

Data for the Christmas Bay System, Brazoria County, Texas, February 1999-March 2000

Open-File Report 02-082

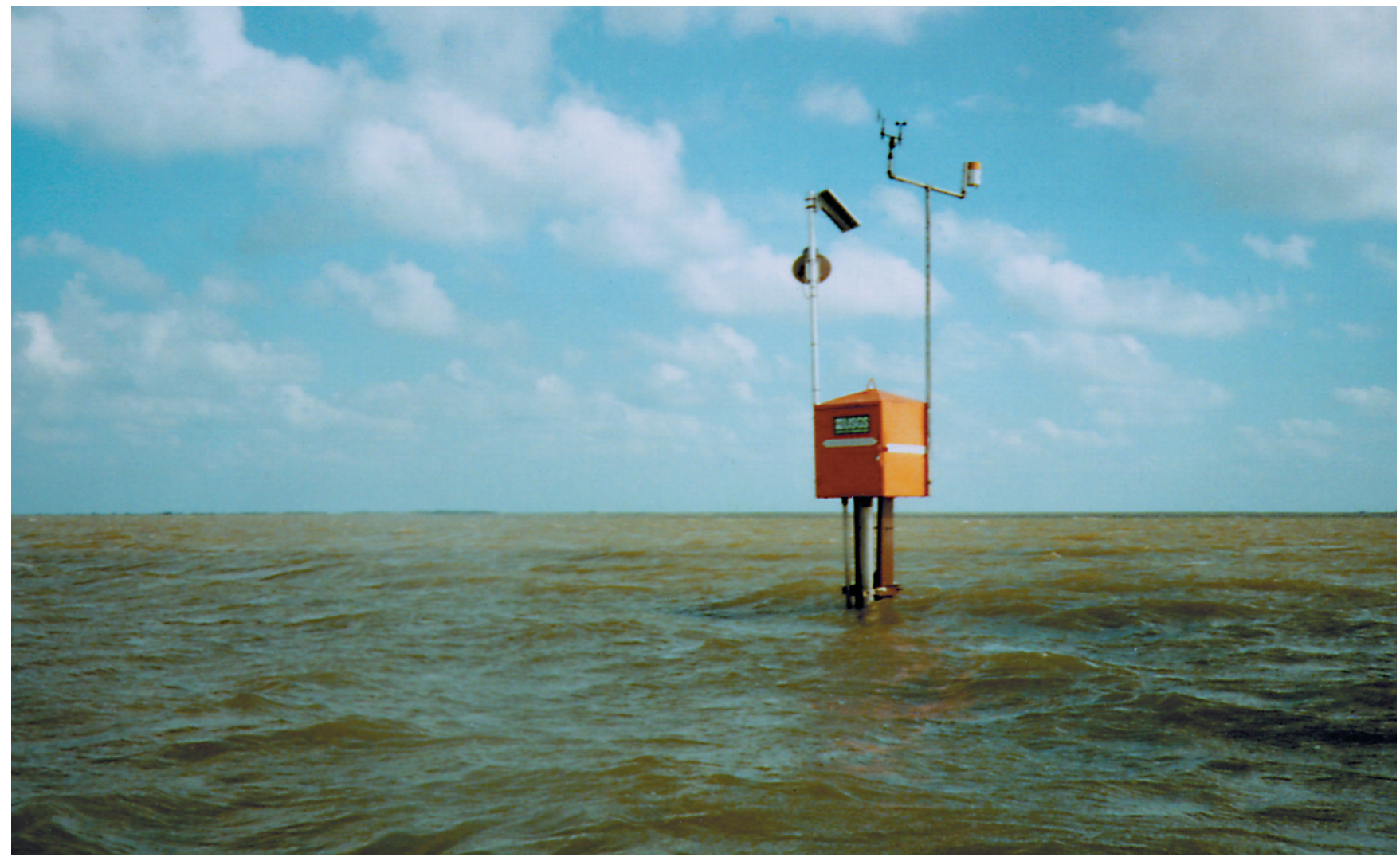

U.S. Department of the Interior U.S. Geological Survey 


\section{Cover:}

Gage at Christmas Bay station (photograph by by Jeffery W. East, December 1998). 
U.S. Department of the Interior

U.S. Geological Survey

\section{Hydrologic, Water-Quality, and Sediment-Quality Data for the Christmas Bay System, Brazoria County, Texas, February 1999-March 2000}

By Jeffery W. East

\section{U.S. GEOLOGICAL SURVEY}

Open-File Report 02-082

In cooperation with the Houston-Galveston Area Council 


\section{U.S. DEPARTMENT OF THE INTERIOR}

Gale A. Norton, Secretary

\section{U.S. GEOLOGICAL SURVEY}

Charles G. Groat, Director

Any use of trade, product, or firm names is for descriptive purposes only and does not imply endorsement by the U.S. Government.

For additional information write to

\section{District Chief}

U.S. Geological Survey

8027 Exchange Dr.

Austin, TX 78754-4733

E-mail: dc_tx@usgs.gov

Copies of this report can be purchased from

U.S. Geological Survey

Information Services

Box 25286

Denver, CO 80225-0286

E-mail: infoservices@usgs.gov 


\section{CONTENTS}

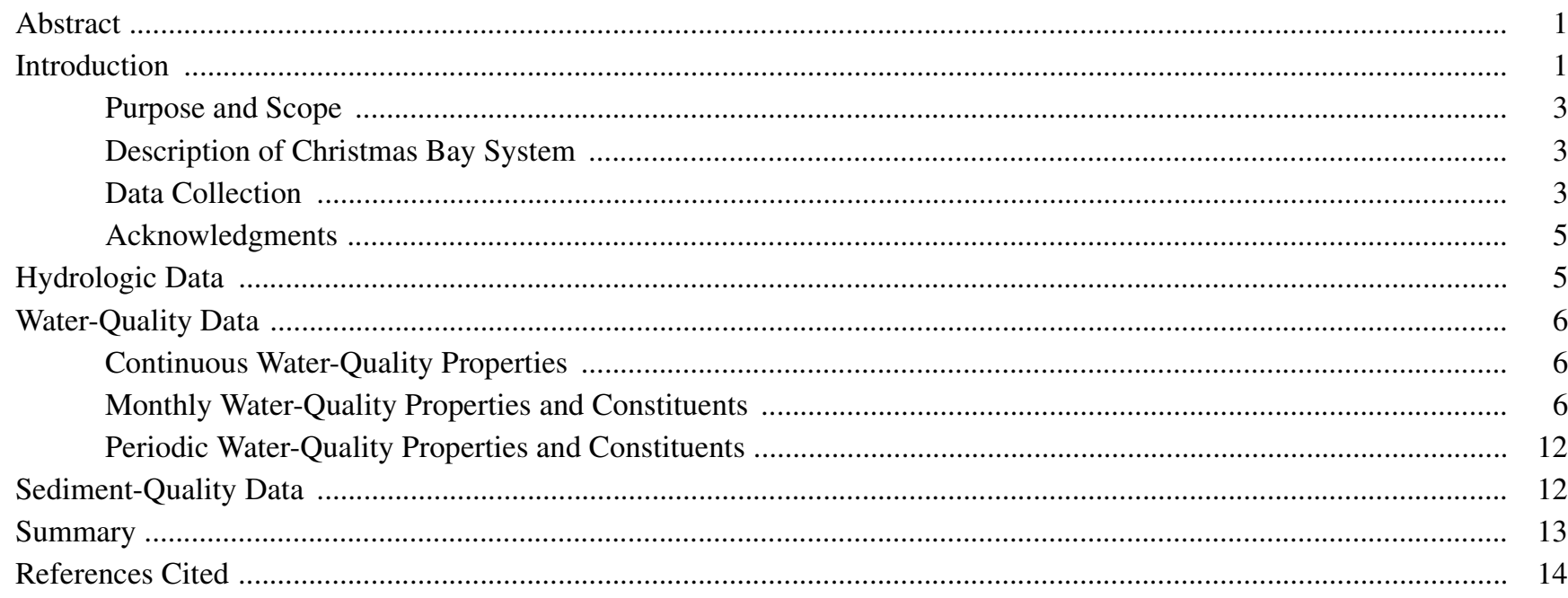

\section{FIGURES}

1. Map showing Christmas Bay system and locations of data-collection stations ……...................................... 2

2. Map showing locations of data-collection stations outside Christmas Bay system .......................................... 4

3. Boxplots showing distribution of gage height at Christmas Bay and tidal altitude at the Galveston Pleasure Pier, February 1999-February 2000

4. Graph showing rainfall at National Weather Service gage, Scholes Airfield, Galveston, February 1999_ February 2000

5. Diagram showing wind speed and direction at Christmas Bay and Freeport, February 1999-February 2000 ....

6. Boxplots showing distribution of (a) specific conductance, (b) $\mathrm{pH}$, (c) water temperature, and (d) dissolved oxygen in Christmas Bay, February 1999-January 2000

\section{TABLES}

1. Summary of data-collection activities in the Christmas Bay system, February 1999-March 2000 ................... 5

2. Maximum, minimum, and mean gage heights in Christmas Bay, February 1999-February 2000 .................... 15

3. Maximum, minimum, and mean specific conductance, $\mathrm{pH}$, water temperature, and dissolved oxygen in Christmas Bay, February 1999-February 2000

4. Monthly and periodic water-quality data in Christmas, Bastrop, and Drum Bays, February 1999January 2000

5. Semivolatile organic compounds in samples collected using a semipermeable membrane device deployed in Christmas Bay, February 29-March 29, 2000

6. Grain size in bed-sediment samples collected in Christmas, Bastrop, and Drum Bays, November 30, 1999

7. Carbon and major and trace elements in bed-sediment samples collected in Christmas, Bastrop, and Drum Bays, November 30, 1999

8. Semivolatile organic compounds (mostly polycyclic aromatic hydrocarbons) in bed-sediment samples collected in Christmas, Bastrop, and Drum Bays, November 30, 1999

9. Organochlorine pesticides in bed-sediment samples collected in Christmas, Bastrop, and Drum Bays, November 30, 1999 


\title{
Hydrologic, Water-Quality, and Sediment-Quality Data for the Christmas Bay System, Brazoria County, Texas, February 1999-March 2000
}

\author{
By Jeffery W. East
}

\section{Abstract}

The Christmas Bay system is a group of three small secondary bays (Christmas, Bastrop, and Drum Bays) at the southwestern end of the Galveston Bay estuarine system in Brazoria County, Texas. During February 1999-March 2000, hydrologic, water-quality, and sedimentquality data were collected from each of the three bays to establish baseline conditions. Gage-height fluctuations closely matched open-water tidal fluctuations. Rainfall during February 1999-February 2000 was about 20 percent below the annual average. Specific conductance, $\mathrm{pH}$, water temperature, and dissolved oxygen monitored at 30-minute intervals in Christmas Bay for 13 months showed seasonal variations typical of monitoring stations on the Texas Gulf Coast. Prevailing winds were from the southeast. Monthly water-quality sampling for 13 months showed that in each of the three bays concentrations of major ions were small, and most nutrient concentrations were at or less than minimum reporting levels; indicator bacteria counts were consistently higher in samples collected from Drum Bay. Several trace elements (sampled twice) were detected in small concentrations. The only organochlorine pesticides (sampled once) that were greater than minimum reporting levels were atrazine, deethylatrazine, metolachlor, and simazine. During February 29-March 29, 2000, three semipermeable membrane devices were deployed at the Christmas Bay monitoring station. Seven of 77 semivolatile organic compounds analyzed in the lipids from the devices were detected in minute amounts. Analyses of surficial bed sediment sampled once in each of the three bays yielded detections of a number of semivolatile organic compounds; all concentrations were less than 10 micrograms per liter and much less than the respective benchmark concentration for those compounds that have had a benchmark concentration established for the protection of aquatic life.

\section{INTRODUCTION}

The Christmas Bay system is a group of three small secondary bays at the southwestern end of the Galveston Bay estuarine system in Brazoria County, Texas. The three-bay system, which comprises Christmas, Bastrop, and Drum Bays, lies inland of Follets Island, a barrier island southwest of West Bay and San Luis Pass (fig. 1). Christmas Bay is recognized as "a near-pristine, 5,660-acre habitat" with "no known water-quality problems, nor indications of potential water-quality problems" (McFarlane, 1991, p. 1). However, McFarlane (1991, p. 1) also indicates that "the current monitoring program is inadequate" and suggests that monthly sampling to "establish baseline conditions" be undertaken. Mitchell and Windsor (1991, p. 8) state that, although Christmas Bay "remains one of the most pristine areas in the Galveston Bay system," the area "faces some of the same potentially significant risks to its water and habitat quality that threaten the entire bay system."

Numerous species of birds, fish, crustaceans, and mollusks inhabit the Christmas Bay system. These biota include seven endangered species of birds and an endangered species of sea turtle. Numerous flora also occur in the Christmas Bay system and include several species of seagrass that serve as prime spawning grounds for crustaceans and finfish. One seagrass habitat has decreased about 36 percent since 1956 (McFarlane, 1991, p. 2).

Previous water-resource investigations of the area have been reconnaissance in nature, including a contaminant study of benthic macroinvertebrates, finfishes, 


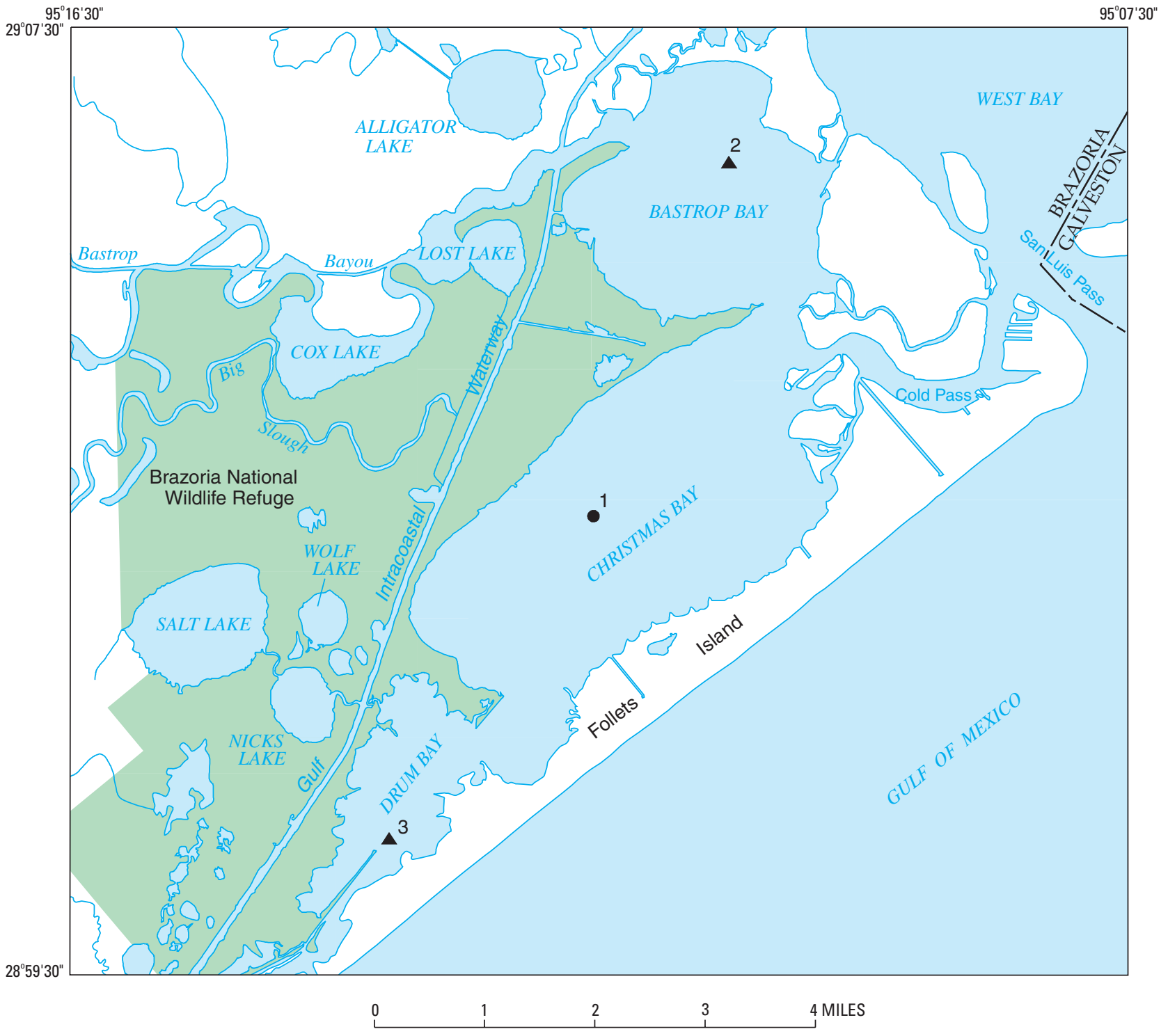

\section{EXPLANATION}

1. USGS monitoring and sampling station and site identification number (table 1)

$2 \quad$ USGS sampling station and site identification number (table 1)

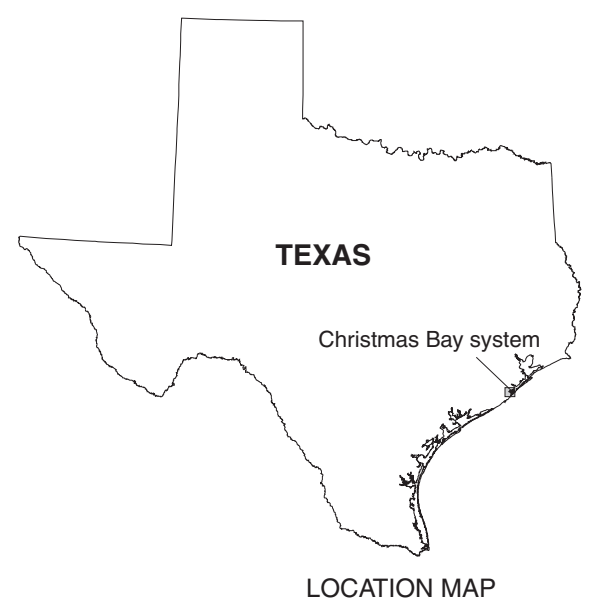

Figure 1. Christmas Bay system and locations of data-collection stations. 
and sediment in Christmas Bay (Sager, 1995). The Texas Natural Resource Conservation Commission (TNRCC) collects water-quality and sedimentcontaminant data from each of the bays on a quarterly basis as part of the State's water-quality monitoring program (Texas Natural Resource Conservation Commission, 1994).

During February 1999-March 2000, the U.S. Geological Survey (USGS) collected hydrologic, waterquality, and sediment-quality data in cooperation with the Houston-Galveston Area Council to establish baseline conditions in the Christmas Bay system. This datacollection effort was part of the TNRCC Clean Rivers Program.

\section{Purpose and Scope}

The purpose of this report is to document hydrologic, water-quality, and sediment-quality data collected from Christmas Bay, Bastrop Bay, and Drum Bay during February 1999-March 2000. Graphs and tables present hydrologic parameters that were monitored in Christmas Bay continuously (30-minute intervals) for 13 months. Tables list water-quality properties and constituents sampled monthly in all three bays for 1 year, trace elements sampled twice, and soluble pesticides sampled once. In addition, tables list the results of analyses for semivolatile organic compounds (SVOCs) extracted from three semipermeable membrane devices (SPMDs) deployed at the Christmas Bay monitoring station for 1 month and the results of analyses of bottom sediment in each bay sampled once.

\section{Description of Christmas Bay System}

Christmas Bay lies between Bastrop Bay and Drum Bay (fig. 1) at the southwestern end of the Galveston Bay estuarine system. Christmas Bay and Bastrop Bay exhibit similar bathymetry, with average depths of about 2.5 feet. Drum Bay is shallower, with average depths of about 1.5 feet. The three-bay system is connected to the Gulf of Mexico through Cold Pass and San Luis Pass.

The three bays are bounded on the west by the 12,200-acre Brazoria National Wildlife Refuge. Christmas Bay is included in the Christmas Bay Coastal Preserve, part of the Texas General Land Office/Texas Parks and Wildlife Department Coastal Preserves Program. The Christmas Bay Coastal Preserve is also within the jurisdiction of the Galveston Bay Estuary Program of the TNRCC.
Freshwater inflows to Christmas Bay and adjacent bays come almost exclusively from the Bastrop Bayou watershed (Texas Natural Resource Conservation Commission, 1994). The 58.7-squaremile watershed (McFarlane, 1991, p. 48) is mostly cultivated and is crisscrossed by farms and drainage canals. Agricultural runoff and septic tanks in the watershed are a "concern" regarding water quality in Christmas Bay (Mitchell and Windsor, 1991). Other activities in the watershed that could potentially affect water quality include oil and gas drilling and salt-dome injection by way of wells. The city of Danbury is wholly within the watershed, and the cities of Angleton and Lake Jackson are partially in the basin (fig. 2).

Another hydrodynamic input to the Christmas Bay system is the Gulf Intracoastal Waterway (GIWW). The GIWW makes a path through the wildlife refuge, across the southern part of the Bastrop Bayou watershed and the Christmas Bay system, from southwest to northeast (fig. 1). The dredged depth of the GIWW is maintained at 12 feet, effectively interrupting the overland flow of freshwater to the three bays. The effect of any decrease in freshwater inflow on the Christmas Bay system is unknown.

Additional development on the Christmas Bay shoreline could pose a threat to the quality of this ecosystem. The Texas Parks and Wildlife Department has undeveloped land holdings on Follets Island and the south shore of Christmas Bay (485 acres) for a future Christmas Bay State Park. The presence of about 100 cabins in the bay system potentially could affect water quality because many of the cabins do not use septic systems. Thus, some waste might discharge directly into the bay waters (McFarlane, 1991, p. 11).

\section{Data Collection}

To characterize baseline conditions in the threebay system spatially and temporally, hydrologic parameters (gage height, rainfall ${ }^{1}$, and wind speed and direction) were monitored in Christmas Bay continuously (30-minute intervals) during February 1999-January 2000. Water-quality properties (specific conductance, $\mathrm{pH}$, temperature, and dissolved oxygen) and constituents (nutrients, major ions, phytoplankton, indicator bacteria, and suspended sediment) were sampled in all

\footnotetext{
${ }^{1}$ After the data-collection period, it was discovered that the Christmas Bay rain gage had not been functioning properly, thus the rainfall data were not accurate. Data from a National Weather Service rain gage at Scholes Airfield, Galveston, can be substituted.
} 


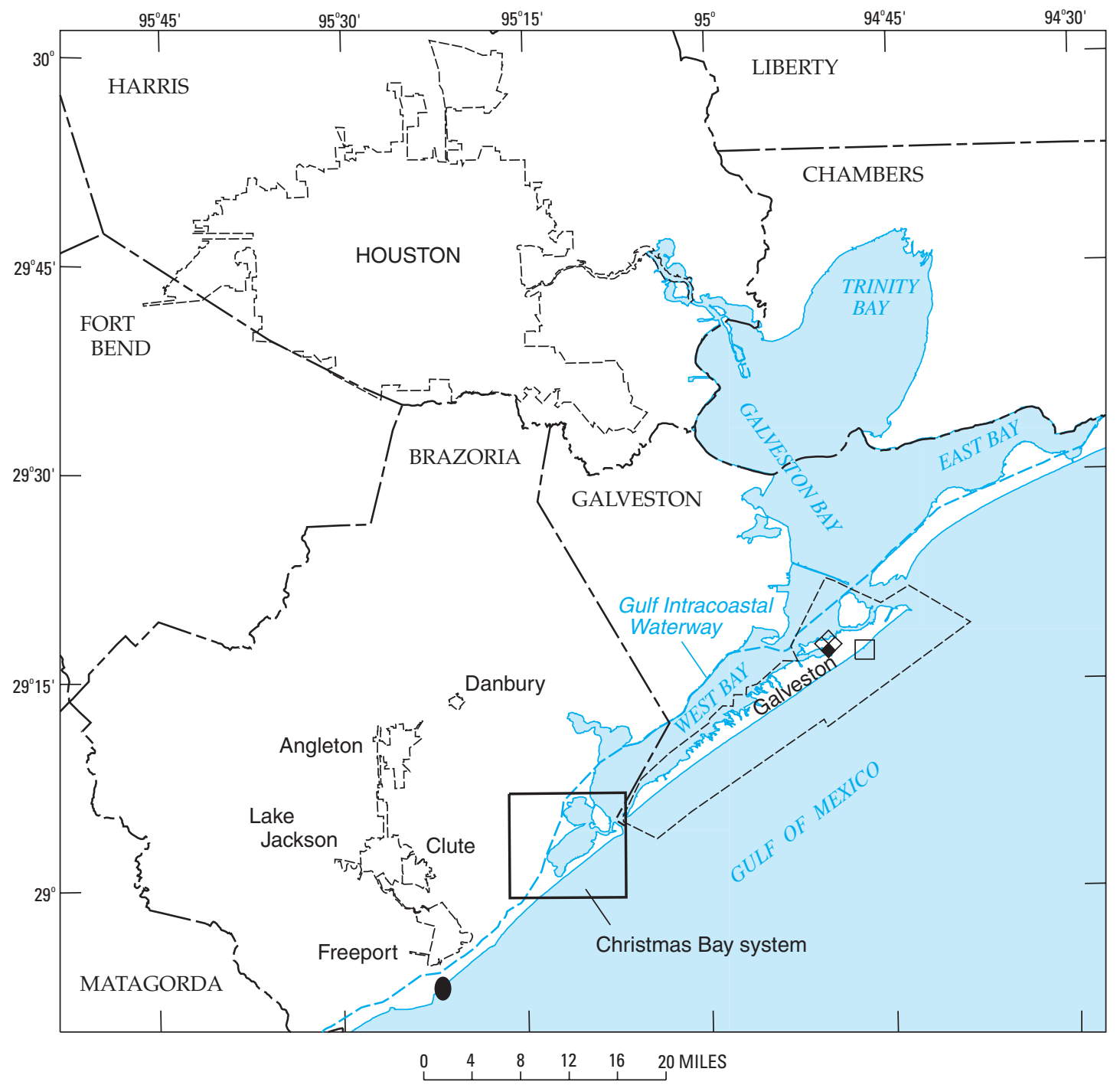

\section{EXPLANATION}

National Oceanic and Atmospheric Administration tide station

National Weather Service rain gage

- Texas Coastal Ocean Observation Network wind station

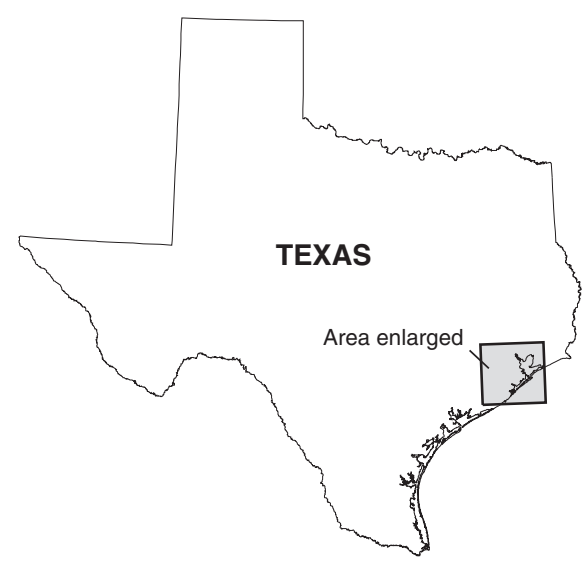

LOCATION MAP

Figure 2. Locations of data-collection stations outside Christmas Bay system. 
Table 1. Summary of data-collection activities in the Christmas Bay system, February 1999-March 2000

\begin{tabular}{|c|c|c|c|c|}
\hline \multirow{2}{*}{ Station name } & \multirow{2}{*}{$\begin{array}{l}\text { Site identi- } \\
\text { fication no. } \\
\text { (fig. 1) }\end{array}$} & \multicolumn{2}{|c|}{ Location } & \multirow{2}{*}{ Data-collection activity } \\
\hline & & Latitude & Longitude & \\
\hline Christmas Bay & 1 & $29^{\circ} 03^{\prime} 08^{\prime \prime}$ & $95^{\circ} 11^{\prime} 48^{\prime \prime}$ & $\begin{array}{l}\text { Continuous hydrologic-parameter monitoring } \\
\text { Continuous water-quality-property monitoring } \\
\text { Monthly water-quality sampling } \\
\text { Periodic water-quality sampling } \\
\text { 30-day semipermeable membrane device } \\
\text { deployment } \\
\text { One-time sediment-quality sampling }\end{array}$ \\
\hline Bastrop Bay & 2 & $29^{\circ} 05^{\prime} 56^{\prime \prime}$ & $95^{\circ} 10^{\prime} 35^{\prime \prime}$ & $\begin{array}{l}\text { Monthly water-quality sampling } \\
\text { Periodic water-quality sampling } \\
\text { One-time sediment-quality sampling }\end{array}$ \\
\hline Drum Bay & 3 & $29^{\circ} 00^{\prime} 35^{\prime \prime}$ & $95^{\circ} 13^{\prime} 38^{\prime \prime}$ & $\begin{array}{l}\text { Monthly water-quality sampling } \\
\text { Periodic water-quality sampling } \\
\text { One-time sediment-quality sampling }\end{array}$ \\
\hline
\end{tabular}

three bays monthly during February 1999-January 2000; trace elements were sampled twice and soluble pesticides once during the same period. SVOCs were extracted from three SPMDs deployed at the Christmas Bay monitoring station during February 29-March 29, 2000; and bottom sediment in each bay was sampled November 30, 1999, for grain size, major and trace elements, carbons, SVOCs (mostly polycyclic aromatic hydrocarbons [PAHs]), and organochlorine pesticides. Data-collection activities are summarized in table 1.

In addition to environmental samples, quality assurance (QA) samples also were collected. Depending on the constituent, QA samples consisted of equipment blanks, field blanks, laboratory blanks, split samples, replicate samples, and laboratory matrix spikes.

\section{Acknowledgments}

The author thanks Carl Masterson, Todd Running, Patrick Horton, and Karen Brettschneider of the Houston-Galveston Area Council for assistance provided throughout the period of data collection. Gary Mitchell, formerly of the Houston-Galveston Area Council, also assisted during the planning stages of data collection. Also, the author acknowledges the Texas Parks and Wildlife Department for permission to install and operate equipment in Christmas Bay.

\section{HYDROLOGIC DATA}

Because of the proximity of the Christmas Bay system to the Gulf of Mexico, as well as the relatively shallow average depths ( 1.5 to 2.5 feet), hydrologic conditions are factors to consider when characterizing the system. The Christmas Bay hydrologic monitoring station consisted of a gage house that contained a submersible pressure transducer to measure tidal gage height, a tipping-bucket rain gage to collect rainfall, and an ultrasonic wind anemometer to measure wind speed and direction. In addition, a multiprobe waterquality monitor was installed at the station to measure the four water-quality properties listed previously. Readings from each sensor were recorded electronically at 30-minute intervals by a data-collection platform. Every 4 hours, these data were transmitted by way of geostationary operational-environmental satellite (GOES) to the USGS National Water Information System database. The data were thus monitored on a near real-time basis. 
Table 2 (at end of report) lists the daily maximum, minimum, and mean gage heights for the 13-month period. Gage height is defined as the water-surface elevation above some datum, usually sea level. The datum in this case was arbitrary because the gage was not referenced to sea level.

The gage-height data for the Christmas Bay station were aggregated by month in boxplots to show monthly range in gage height and variability of gage height (fig. 3). Similar boxplots were developed for tidal altitude data from the National Oceanic and Atmospheric Administration tide station (fig. 2) located at the Galveston Pleasure Pier on the Gulf of Mexico side of Galveston Island. The boxplots for the two stations show a close match between open-water tidal fluctuations and bay water-surface fluctuations.

Total rainfall recorded by the National Weather Service gage at Scholes Airfield, Galveston, (about 20 miles northeast of Christmas Bay) during February 1999-February 2000 (fig. 4) was 34.84 inches (National Weather Service, 2002). The average annual rainfall (1971-2000) for that gage is 44.21 inches. Because rainfall during the 13-month period was well below average (about 20 percent), it is likely that freshwater inflow to the three-bay system, from Bastrop Bayou, also was below normal. However, streamflow data for Bastrop Bayou were unavailable to verify this assumption.

Wind has a substantial effect on gage height in the Christmas Bay system. This is because the "pileup effect of wind is inversely proportional to the depth of the water" (Fisher, 1988, p. 33). Because the Christmas Bay system is relatively shallow, periods of strong wind cause substantial wave "chop" on the water surface and might lead to resuspension of bottom sediment. Wind speed and direction measured at the Christmas Bay station during the 13-month period were used to develop a wind-rose diagram (fig. 5). Wind-rose diagrams group data by wind speed and direction and summarize data for a selected time period. A second wind-rose diagram developed from data recorded by the Texas Coastal Ocean Observation Network (2001) at a station in Freeport, about 15 miles southwest of the Christmas Bay station, shows consistency with the Christmas Bay diagram. The diagrams indicate prevailing winds were from the southeast.

\section{WATER-QUALITY DATA}

\section{Continuous Water-Quality Properties}

During February 1999-January 2000, specific conductance, $\mathrm{pH}$, water temperature, and dissolved oxygen were measured continuously (30-minute intervals) at the monitoring station in Christmas Bay. The depth at the monitor location was about 5 feet at mean tide. These data were monitored using a multiprobe water-quality meter that was deployed near mid-depth, about 2 feet from the bottom of the bay. Statistical summaries of the four properties are listed in table 3 (at end of report). Figure 6 shows boxplots of these data aggregated by month. Seasonal variations in these properties are typical of those observed at USGS stations on the Texas Gulf Coast. In particular, water temperature and dissolved oxygen concentration are inversely related, with periods of smallest dissolved oxygen concentrations occurring in the summer months when water temperatures are highest.

\section{Monthly Water-Quality Properties and Constituents}

Selected water-quality properties and constituents were determined during monthly visits to each of the three bays (table 4, at end of report). Specific conductance, $\mathrm{pH}$, water temperature, and dissolved oxygen were measured in the field. Water-quality constituents that were determined by laboratory analysis include

1. Major ions (calcium, magnesium, potassium, sodium, chloride, fluoride, silica, sulfate) and trace elements (iron, manganese, mercury)

2. Nutrients (ammonia nitrogen, ammonia plus organic nitrogen, nitrite plus nitrate nitrogen, nitrite nitrogen, phosphorus, orthophosphorus)

3. Phytoplankton (biomass, chlorophyll- $a$, chlorophyll- $b$ )

4. Indicator bacteria (fecal coliform, fecal streptococcus)

5. Suspended sediment

Each month, physical landmarks and a portable global positioning system (GPS) unit were used to ensure that samples were collected at the same location in each of the three bays. Water depths at the three sites were relatively shallow (about 2 feet in Drum Bay, 2.5 feet in Bastrop Bay, and 5 feet in Christmas Bay); 


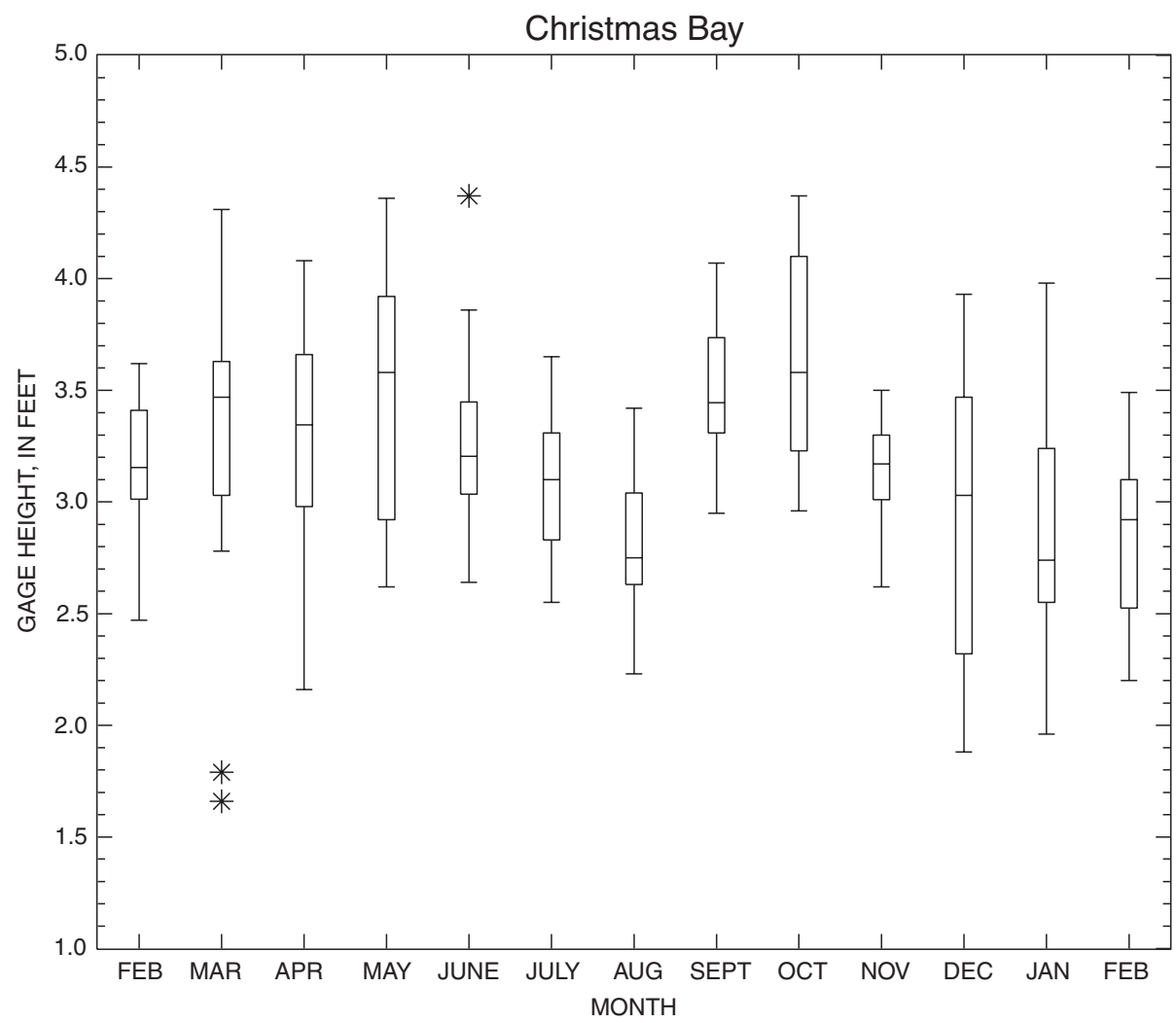

\section{EXPLANATION}

Data value greater than 3.0 times the IQR outside the box

* Data value 1.5 to 3.0 times the IQR outside the box

Largest data value within 1.5 times the IQR above the box

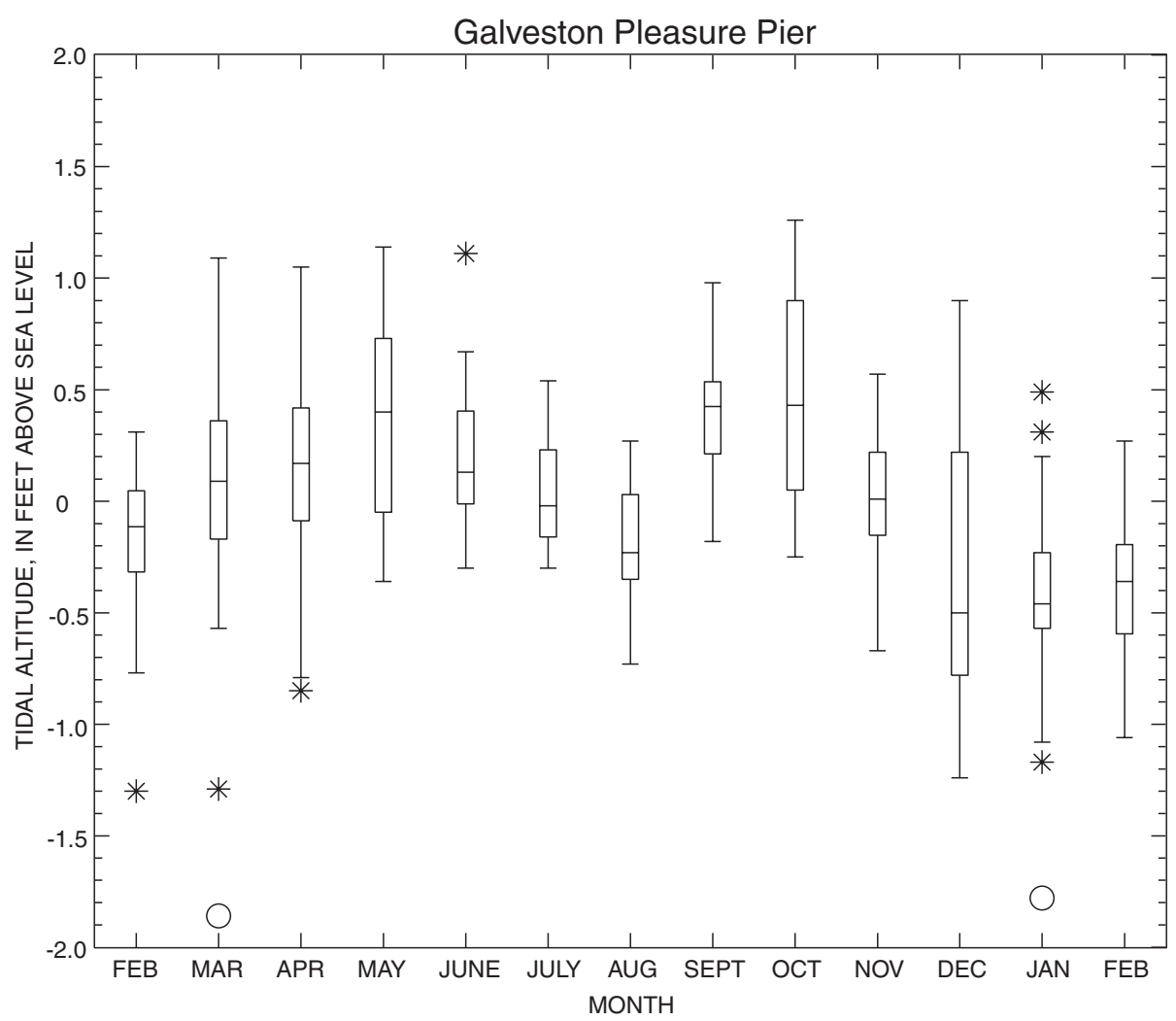

75th percentile

Median (50th percentile) Interquartile

25th percentile

Smallest data value within 1.5 times the IQR below the box range (IQR)

Figure 3. Distribution of gage height at Christmas Bay and tidal altitude at the Galveston Pleasure Pier, February 1999-February 2000. 


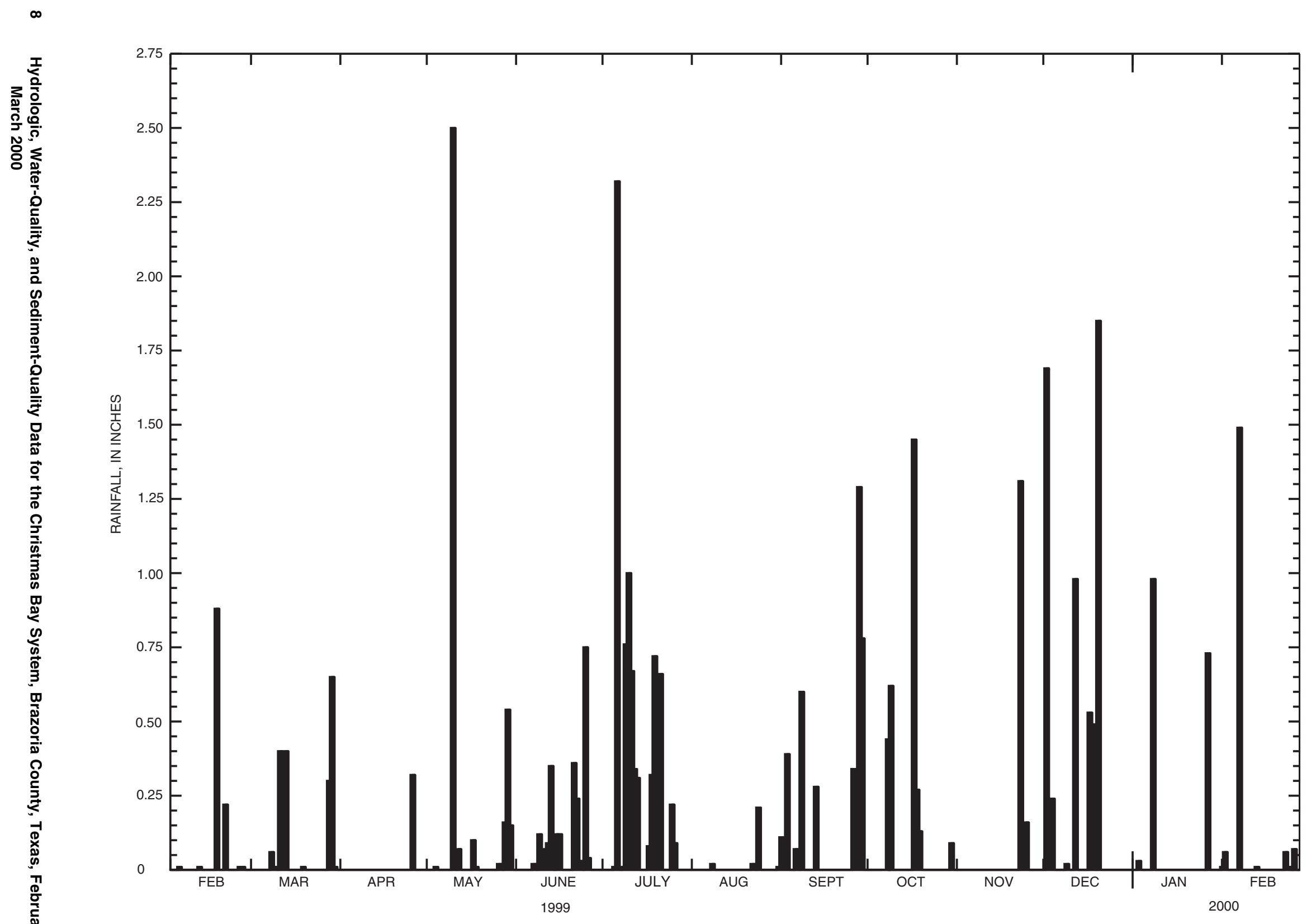

Figure 4. Rainfall at National Weather Service gage, Scholes Airfield, Galveston, February 1999-February 2000. 

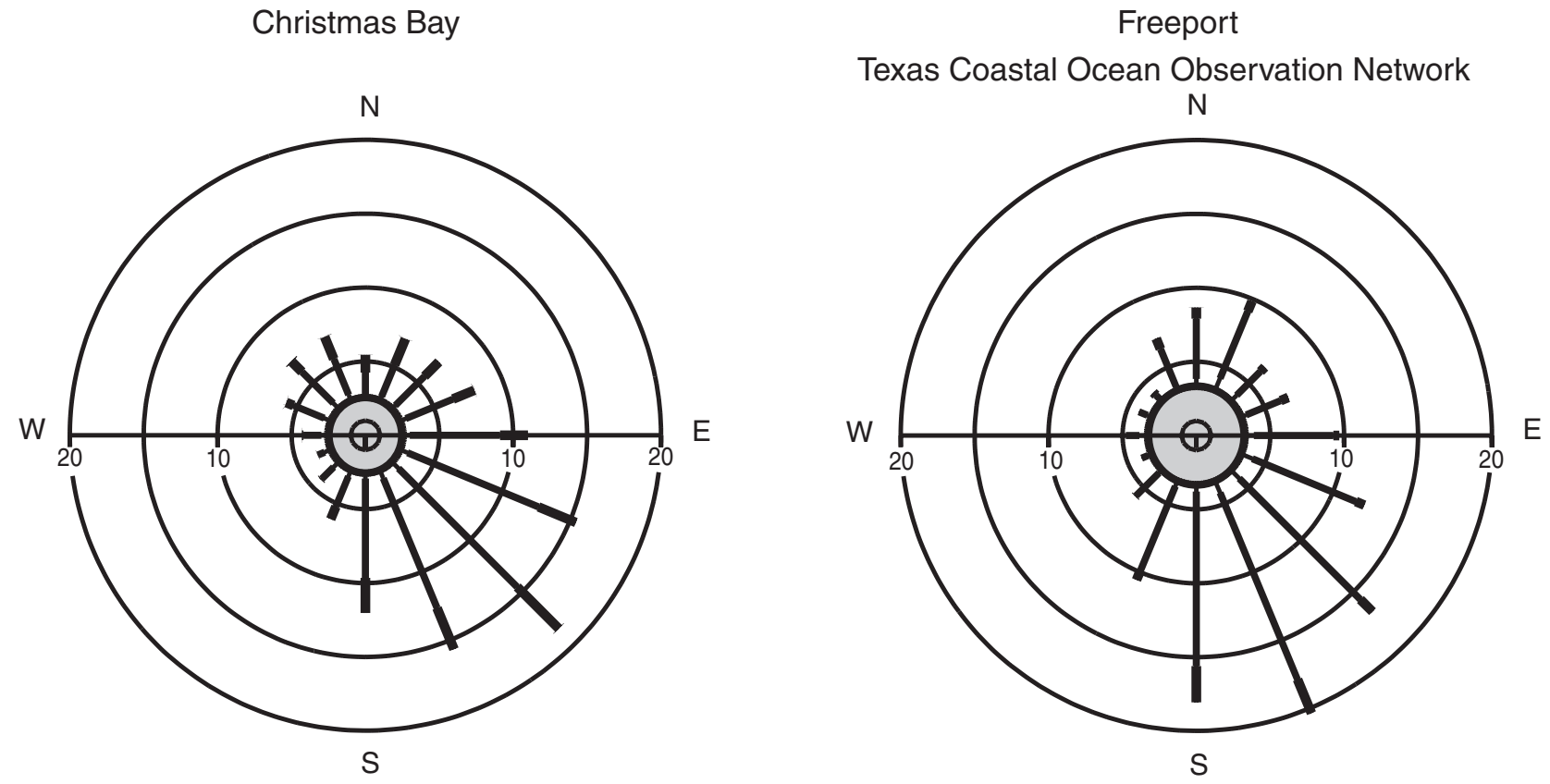

EXPLANATION

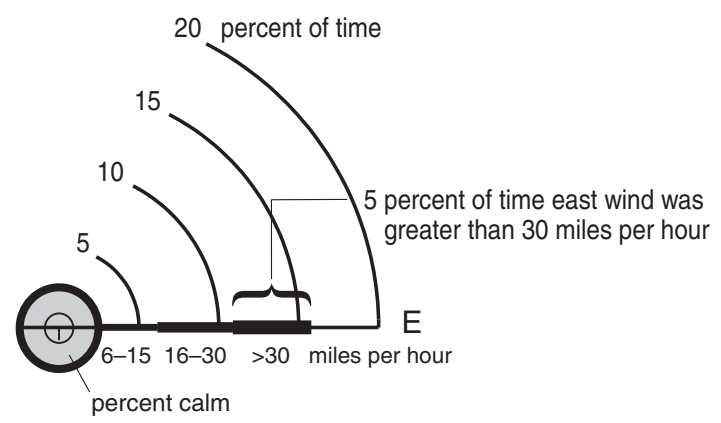

Figure 5. Wind speed and direction at Christmas Bay and Freeport, February 1999-February 2000.

therefore, water samples were collected about 1 foot below the water surface at each site. Because the samples were collected near the surface and the continuous water-quality monitor in Christmas Bay was about 5 feet below the surface, values of specific conductance, $\mathrm{pH}$, water temperature, and dissolved oxygen using the two modes of measurement during site visits might not be comparable.

Standard USGS procedures for sample compositing, filtering, and preservation (Ward and Harr, 1990) were followed. Laboratory analyses for major ions, nutrients, and phytoplankton were done by the USGS National Water Quality Laboratory (NWQL) in Denver, Colo. Analyses for indicator bacteria were done in the
USGS Houston Subdistrict laboratory, and analyses for suspended sediment concentration were done by the USGS Louisiana District sediment laboratory in Baton Rouge, La.

Laboratory analyses for selected constituents, such as iron and manganese, were done using several methods. As a result, the minimum reporting levels varied, as reflected by different "less than" $(<)$ values in table 4.

Results of laboratory analysis show that concentrations of major ions were small in each of the three bays. Most nutrient concentrations were at or less than minimum reporting levels in each of the three bays throughout the data-collection period. Indicator bacteria 


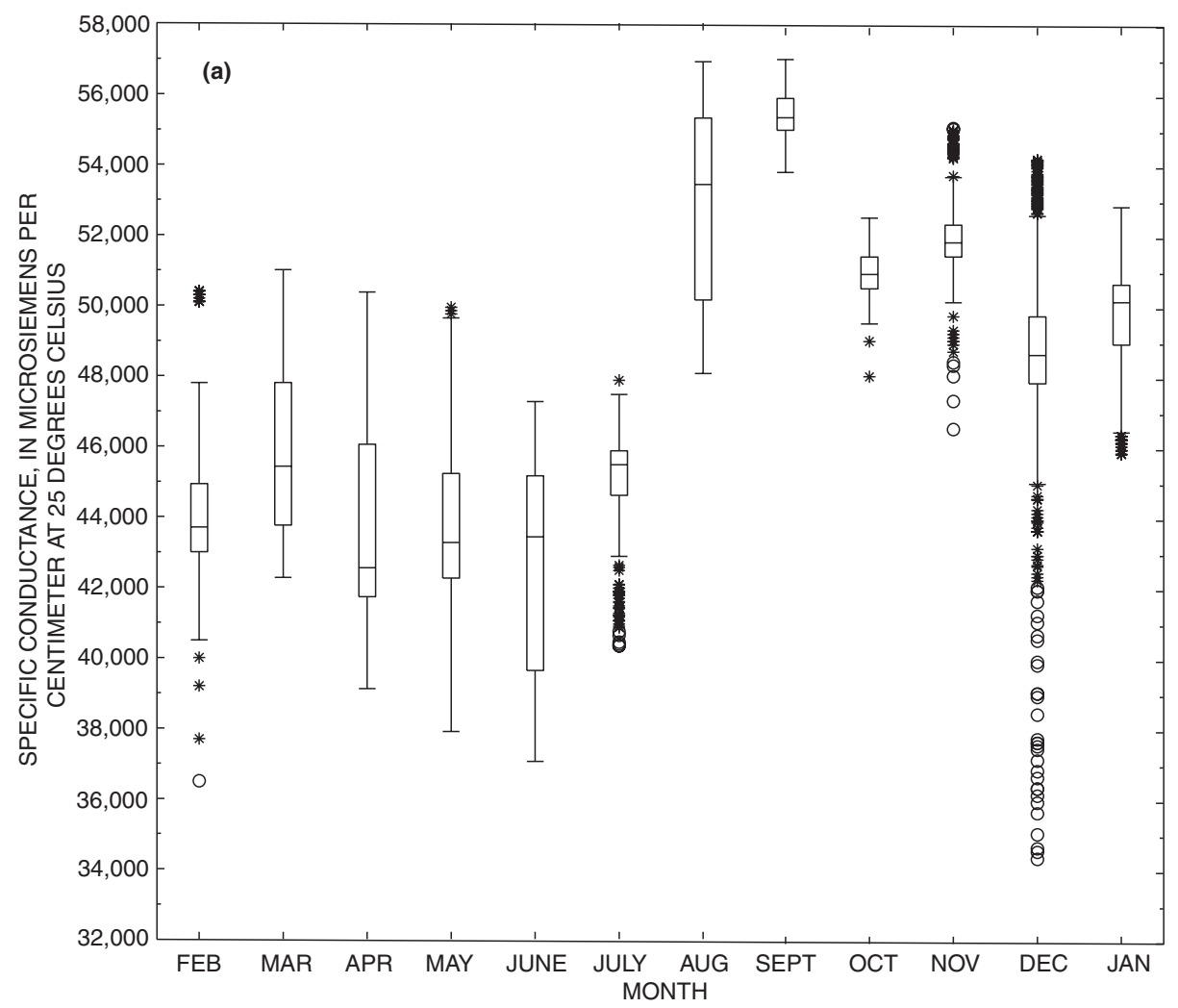

\section{EXPLANATION}

○ Data value greater than 3.0 times the IQR outside the box

* Data value 1.5 to 3.0 times the IQR outside the box

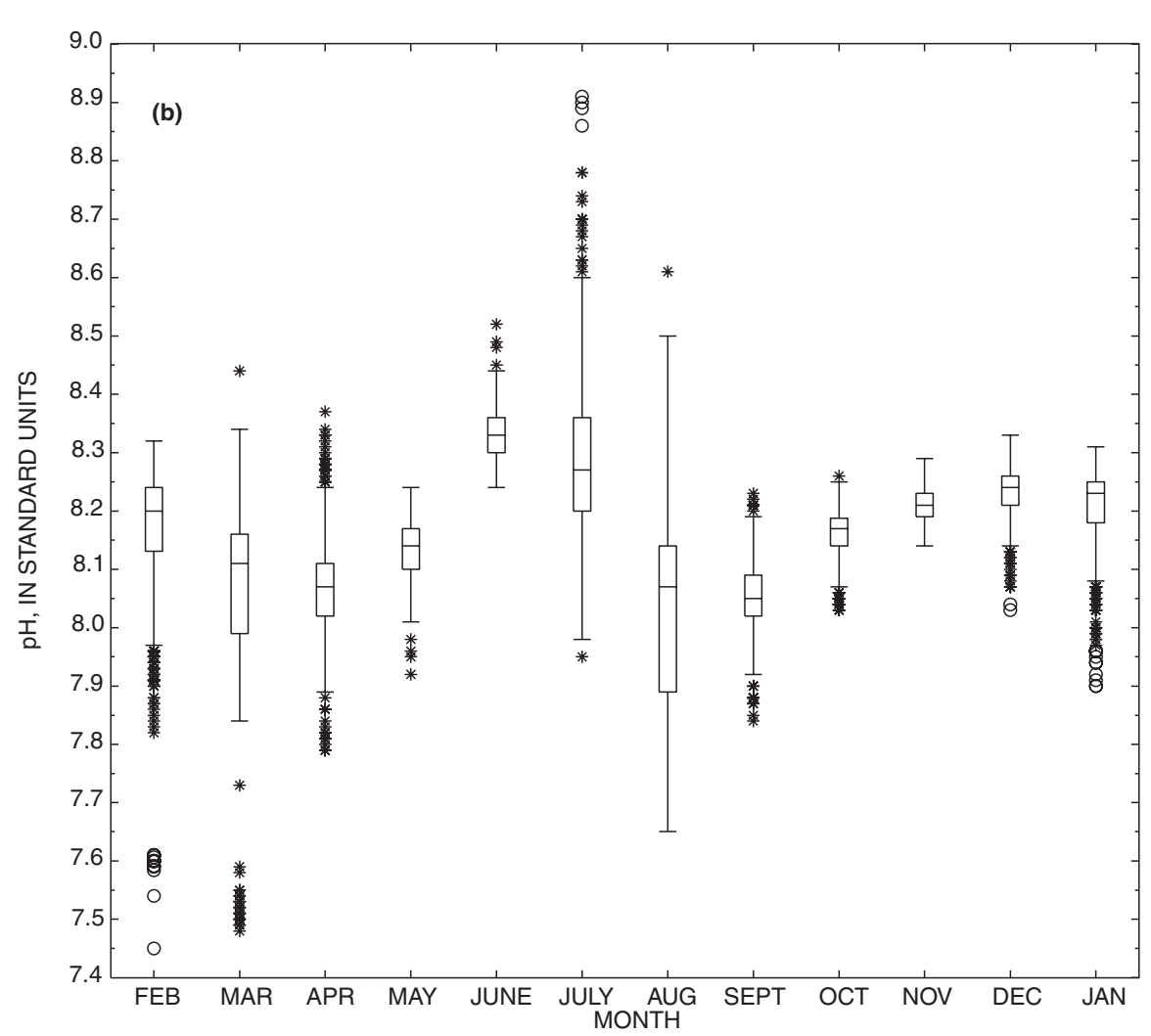

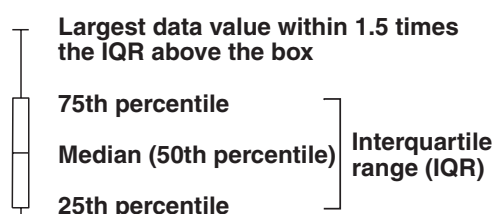

25th percentile

Smallest data value within 1.5 times the IQR below the box

Figure 6. Distribution of (a) specific conductance, (b) $\mathrm{pH},(\mathrm{c})$ water temperature, and (d) dissolved oxygen in Christmas Bay, February 1999-January 2000. 


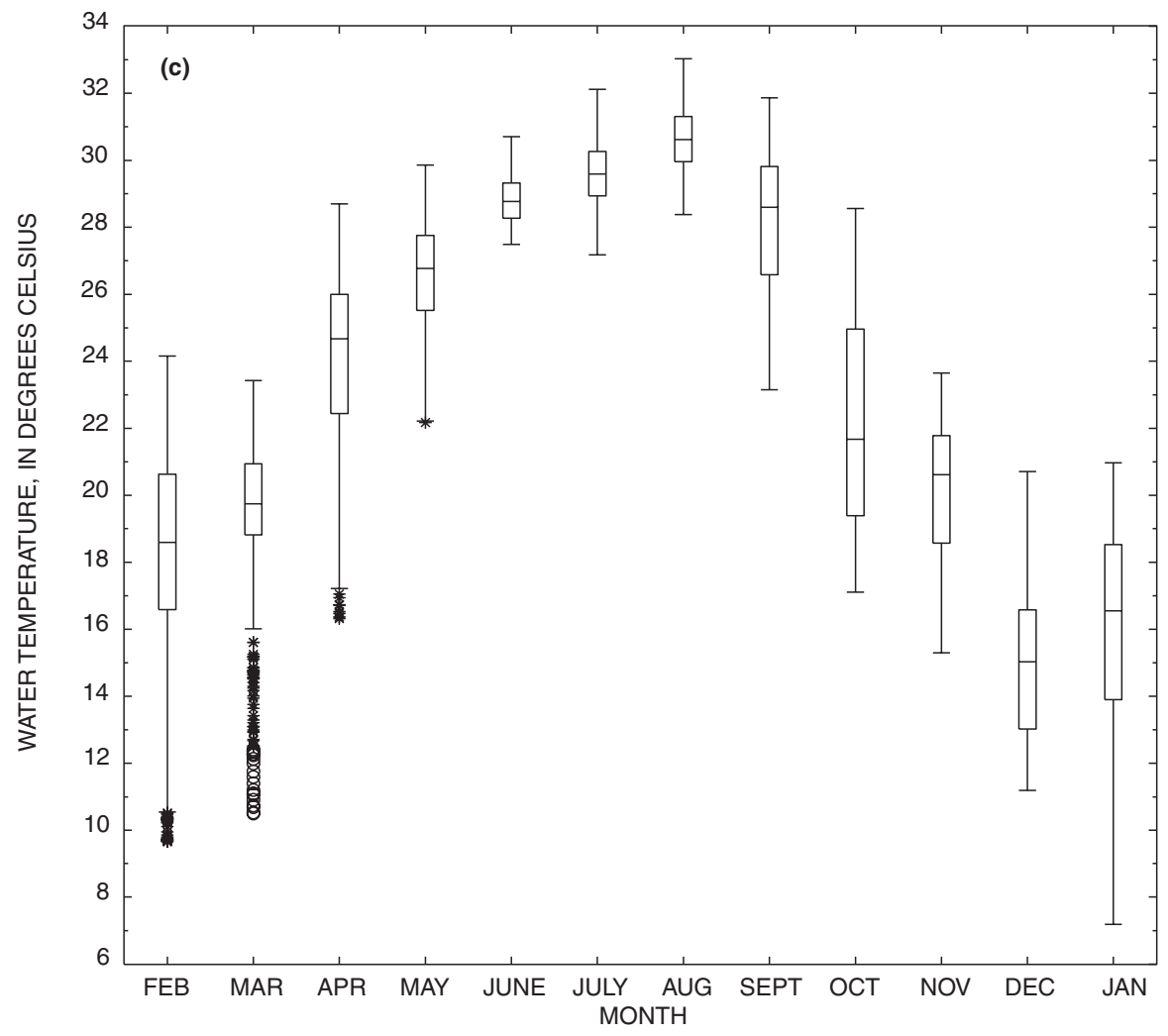

\section{EXPLANATION}

- Data value greater than $\mathbf{3 . 0}$ times the IQR outside the box

* Data value 1.5 to 3.0 times the IQR outside the box

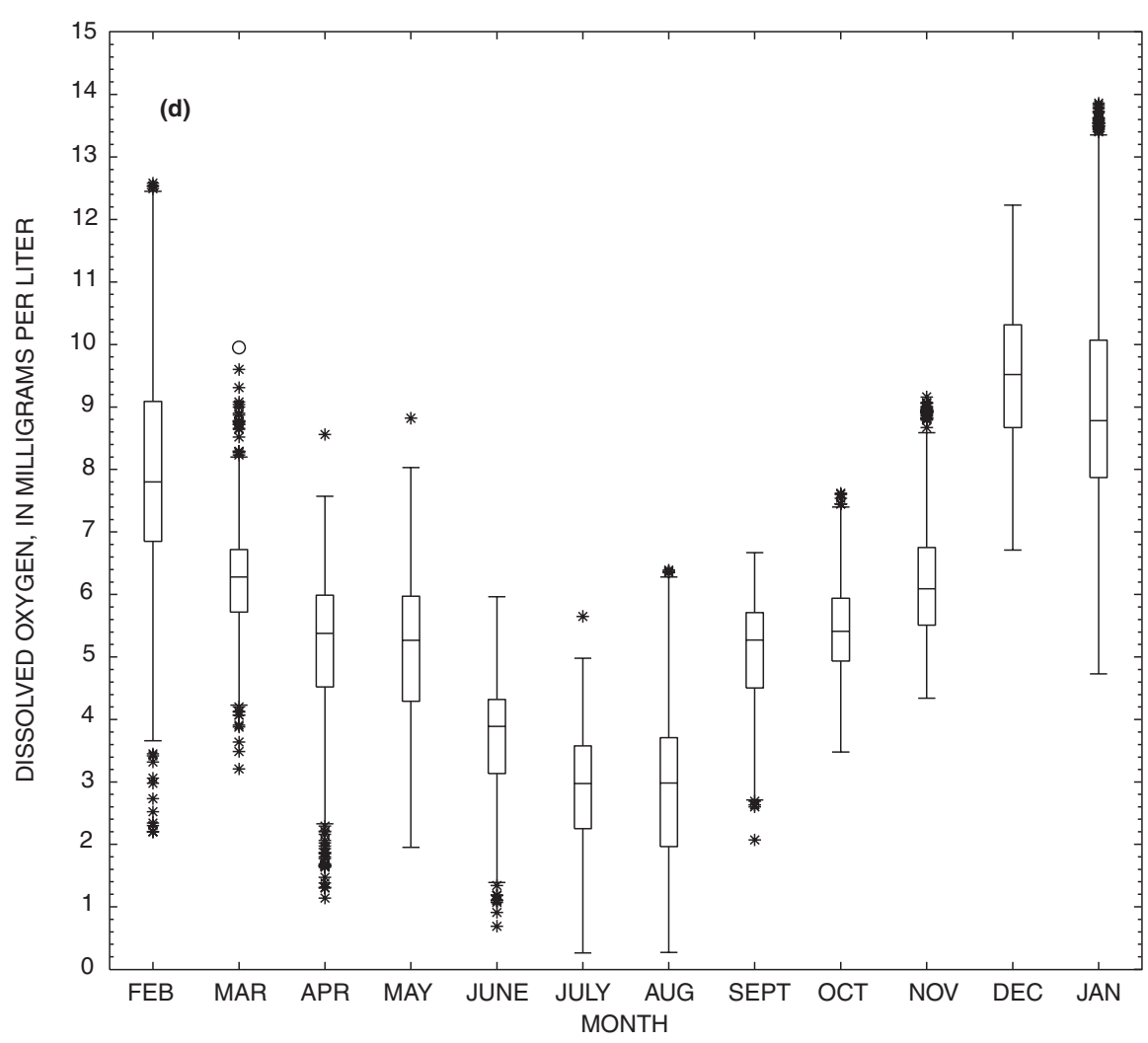

Largest data value within 1.5 times the IQR above the box

75th percentile

Median (50th percentile) Interquartile

25th percentile

Smallest data value within 1.5 times the IQR below the box

Figure 6. Continued. 
counts were consistently higher in samples collected from Drum Bay.

To ensure the quality of these data, various QA samples were collected along with environmental samples. Equipment blanks and field blanks were used to verify the adequacy of cleaning procedures. Split samples were used to determine the analytical precision (reproducibility) for various constituents. Concurrent samples were used to provide a measure of sampling precision (reproducibility) and to indicate spatial or temporal inhomogeneities in the system being sampled. Results of concurrent samples also can reflect differences in sampling, processing, and laboratory analysis. In the Quality Assurance Project Plan, the stated QA objective for sampling and analytical precision was a relative percent difference (RPD) of less than 20 . The RPD of all split and concurrent samples were within the 20-percent objective. If equipment blanks or field blanks were greater than QA limits (two times the minimum reporting level or 10 percent of the environmental value), a remark code of "V" (indicating contamination) precedes the value in table 4 .

\section{Periodic Water-Quality Properties and Constituents}

During the May and December monthly site visits, water samples were collected from each bay and submitted to the NWQL for analysis of the following trace elements: aluminum, antimony, arsenic, barium, beryllium, cadmium, chromium, cobalt, copper, iron, lead, manganese, mercury, molybdenum, nickel, selenium, silver, zinc, and uranium. Results of these analyses are listed in table 4 . Arsenic, barium, chromium, iron, manganese, nickel, and selenium were detected in small concentrations in all three bays; aluminum and zinc also were detected in small concentrations in Christmas and Drum Bays.

During the April monthly site visits, water samples were collected from each bay and submitted to the NWQL for analysis of selected organochlorine pesticides. Results of these analyses are listed in table 4 . The only pesticides that were greater than minimum reporting levels were atrazine and its breakdown product deethylatrazine, metolachlor, and simazine. Concentrations of these four pesticides were tens to hundreds of times less than benchmark concentrations in water established for the protection of aquatic life. Atrazine, metolachlor, and simazine are commonly used herbicides and were three of the four most frequently detected herbicides in the Trinity River Basin during the 1992-95 USGS National Water-Quality Assessment (Land and others, 1998, p. 9).

During February 29-March 29, 2000, three SPMDs were deployed at the Christmas Bay monitoring station (fig. 1). SPMDs are lipid-filled membranes that simulate bioaccumulation occurring in living organisms, such as in the gills of fish. By deploying SPMDs for an extended duration, very small concentrations of constituents in the water can accumulate to a level that can be detected through laboratory analysis. Samples extracted from the SPMDs in Christmas Bay were composited and analyzed for 77 SVOCs. Results from these analyses, along with QA data (field blank, laboratory blank, and laboratory matrix spike), are listed in table 5 (at end of report). The SVOCs detected in uncontaminated samples in concentrations greater than minimum reporting levels were $p$-cresol, 4-chloro-3methylphenol, 2,6-dimethylnaphthalene, 1,6-dimethylnaphthalene, 2,2-biquinoline, Di-n-octylphthalate, benzo $[b]$ fluoranthene, and benzo[ $k]$ fluoranthene. Each of these constituent concentrations was estimated by lab technicians because they were present in such minute amounts. Benchmark concentrations of these seven SVOCs in tissue have not been established for the protection of aquatic life.

The concentrations of phenol and bis(2-ethylhexyl)phthalate were greater in field blanks than in environmental samples. Because the concentrations in the field blanks and environmental samples were of the same order of magnitude, it is impossible to determine whether the source of the SVOCs is the water or the atmosphere. Also, phthalates can be present in the SPMD material (S.L. Marr, U.S. Geological Survey, written commun., 2001). Therefore, whether phenol or bis(2-ethylhexyl)phthalate was present in the water is unknown. Benchmark concentrations of these two SVOCs in tissue have not been established for the protection of aquatic life.

\section{SEDIMENT-QUALITY DATA}

Sediment quality is an integral aspect of water quality because of the transport and fate of chemical constituents associated with sediment; for example, hydrophobic contaminants can be highly bioavailable. To identify the presence of various constituents in bottom sediment, surficial bed-sediment samples were collected from each of the three bays on November 30, 1999, and submitted for laboratory analysis. Samples 
were analyzed for grain size by the USGS Iowa District sediment laboratory and for major and trace elements, carbons, SVOCs (mostly PAHs), and organochlorine pesticides by the NWQL.

Surficial sediment samples were collected using a 15 - by 15 - by 20 -centimeter Wildco box corer (Van Metre and Callender, 1997). The sampler was gently lowered to the bay bottom using a rope and allowed to penetrate the lacustrine sediment. The sampler then was raised abruptly, which actuates jaws in the sampler to close around the sediment, and brought to the surface. The top 3 centimeters of the sample were extruded by sliding the plexiglass liner down onto a piston, which pushes the sample up into an empty liner. The sample then was sliced horizontally and the top 3 centimeters placed into a baked glass jar. Multiple samples were collected and composited in the glass jar before subsampling.

Subsamples for grain-size analysis were taken directly from the glass jar, placed in plastic containers, and submitted to the laboratory. Grain-size data for all three bays, including QA data (split sample), are listed in table 6 (at end of report). Quantitative data are available only for silt and clay. However, it was observed during collection of the samples that the remaining fraction of grain-size materials was composed primarily of sand and shell fragments.

Subsamples for analysis of carbon and major and trace elements were taken from the glass jar and wetsieved through a 0.062-millimeter mesh using native water. The subsamples were collected in 125-milliliter acid-washed Nalgene jars and submitted to the laboratory for analysis. Laboratory results for these constituents are listed in table 7 (at end of report).

Subsamples for SVOC and organochlorine pesticide analysis were taken from the glass jar and wetsieved through a 2-millimeter screen using native water. The subsamples were collected in baked glass jars and submitted to the laboratory for analysis. Laboratory results for 64 SVOCs, including QA samples (split sample, laboratory blank, and laboratory matrix spike), are listed in table 8 (at end of report). Results for 17 organochlorine pesticides, including QA samples (split sample) are listed in table 9 (at end of report).

Those SVOCs (mostly PAHs) detected in sediment samples generally were at minute concentrations, less than 10 micrograms per kilogram. Concentrations of the SVOCs detected, for which benchmark concentrations in sediment have been established for the protection of aquatic life, were tens or hundreds of times less than the respective benchmark concentrations. All pesticides analyzed in bay sediment were less than minimum reporting levels.

\section{SUMMARY}

The Christmas Bay system is a group of three small secondary bays at the southwestern end of the Galveston Bay estuarine system in Brazoria County. Numerous species of birds, fish, crustaceans, and mollusks inhabit the three-bay system. These biota include seven endangered species of birds and an endangered species of sea turtle. Numerous flora also occur in the Christmas Bay system and include several species of seagrass that serve as prime spawning grounds for crustaceans and finfish. During February 1999-March 2000, the USGS collected hydrologic, water-quality, and sediment-quality data to establish baseline conditions in the Christmas Bay system.

Tidal gage height, wind speed, and wind direction were measured at one station in Christmas Bay. Gageheight fluctuations closely matched open-water tidal fluctuations. Total rainfall during February 1999February 2000 at a National Weather Service gage 20 miles northeast of Christmas Bay was about 20 percent below the annual average; thus, freshwater inflow to the three-bay system, from Bastrop Bayou, probably was below normal during the data-collection period. Windspeed and wind-direction data collected at the Christmas Bay station indicate prevailing winds were from the southeast.

Specific conductance, $\mathrm{pH}$, water temperature, and dissolved oxygen were recorded at 30-minute intervals in Christmas Bay during February 1999-January 2000. These data showed seasonal variations that are typical of stations on the Texas Gulf Coast. In particular, water temperature and dissolved oxygen concentration are inversely related, with periods of smallest dissolved oxygen concentrations occurring in the summer months when water temperatures are highest.

Water samples were collected monthly from each of the three bays. Results of laboratory analysis show that concentrations of major ions were small in each of the three bays, and most nutrient concentrations were at or less than minimum reporting levels. Indicator bacteria counts were consistently higher in samples collected from Drum Bay. Water samples collected during the May and December site visits to each bay were analyzed for trace elements; nine trace elements were 
detected in small concentrations in Christmas and Drum Bays and seven in Bastrop Bay.

During the April monthly site visits, water samples were collected from each bay and submitted for analysis of selected organochlorine pesticides. The only pesticides greater than minimum reporting levels were atrazine and its breakdown product deethylatrazine, metolachlor, and simazine. Concentrations of these pesticides were much less than the respective benchmark concentrations in water established for the protection of aquatic life.

During February 29-March 29, 2000, three SPMDs were deployed at the Christmas Bay monitoring station. Seven of 77 SVOCs analyzed in the lipids from the SPMDs were detected in minute amounts.

Surficial bed-sediment samples collected from each of the three bays on November 30, 1999, were analyzed for major and trace elements, carbon, 64 SVOCs (mostly PAHs), and 17 organochlorine pesticides. Concentrations of SVOCs detected were less than 10 micrograms per liter. Concentrations of those SVOCs for which a benchmark concentration in sediment has been established for the protection of aquatic life were much less than the respective benchmark.

\section{REFERENCES CITED}

Fisher, J.C., 1988, Hydrologic data for the Salt Bayou estuary near Sabine Pass, Texas, October 1984 to March 1986, U.S. Geological Survey Open-File Report 88-499, $128 \mathrm{p}$.

Land, L.F., Moring, J.B., Van Metre, P.C., Reutter, D.C., Mahler, B.J., Shipp, A.A., and Ulery, R.L., 1998, Water quality in the Trinity River Basin, Texas, 1992-95:

U.S. Geological Survey Circular 1171, 39 p.

McFarlane, R.W., 1991, An environmental inventory of the Christmas Bay Coastal Preserve: Webster, Tex., Galveston Bay National Estuary Program, GBNEP-7, $95 \mathrm{p}$.

Mitchell, Gary, and Windsor, Duane, 1991, Regulatory effectiveness study for the Christmas Bay Coastal Preserve: Webster, Tex., Galveston Bay National Estuary Program, GBNEP-14, 188 p.

National Weather Service, 2002, Local monthly climate sites data, Scholes Airfield, Galveston: Accessed February 19, 2002, at URL http://www.srh.noaa.gov/HGX/index3/climate3.htm

Sager, David, 1995, An assessment of contaminant concentrations in estuarine resources of selected reference bays of coastal Texas: Texas Parks and Wildlife Department, EQTS-1995-003.

Texas Coastal Ocean Observation Network, 2001, Conrad Blucher Institute, Division of Nearshore Research, data query: Accessed March 12, 2001, at URL http://dco.cbi.tamucc.edu/pquery/056

Texas Natural Resource Conservation Commission, 1994, The State of Texas water-quality inventory-Volume 2 (12th ed.): State/Federal Report 11.

Van Metre, P.C., and Callender, Edward, 1997, Water-quality trends in White Rock Creek Basin from 1912-1994 identified using sediment cores from White Rock Lake reservoir, Dallas, Texas: Journal of Paleolimnology, v. 17, p. 239-249.

Ward, J.R., and Harr, C.A., 1990, Methods for collection and processing of surface-water and bed-material samples for physical and chemical analysis: U.S. Geological Survey Open-File Report 90-140, 71 p. 
Table 2. Maximum, minimum, and mean gage heights in Christmas Bay, February 1999-February 2000

GAGE HEIGHT, IN FEET

\begin{tabular}{|c|c|c|c|c|c|c|c|c|c|c|c|c|}
\hline \multirow[t]{2}{*}{ DAY } & MAX & MIN & MEAN & MAX & MIN & MEAN & MAX & MIN & MEAN & MAX & MIN & MEAN \\
\hline & \multicolumn{3}{|c|}{ FEBRUARY 1999} & \multicolumn{3}{|c|}{ MARCH 1999} & \multicolumn{3}{|c|}{ APRIL 1999} & \multicolumn{3}{|c|}{ MAY 1999} \\
\hline 1 & 3.43 & 2.52 & 3.01 & 3.27 & 2.33 & 2.80 & 3.75 & 3.23 & 3.56 & 4.85 & 3.82 & 4.36 \\
\hline 2 & 3.58 & 2.78 & 3.25 & 3.53 & 2.77 & 3.19 & 3.93 & 3.35 & 3.71 & 4.45 & 3.57 & 4.07 \\
\hline 3 & 3.63 & 3.12 & 3.42 & 3.33 & 2.65 & 2.92 & 4.39 & 3.59 & 4.07 & 3.96 & 3.30 & 3.67 \\
\hline 4 & 3.76 & 3.19 & 3.42 & 4.05 & 2.75 & 3.41 & 4.37 & 3.57 & 4.08 & 4.40 & 3.27 & 3.92 \\
\hline 5 & 3.83 & 3.42 & 3.62 & 4.13 & 3.36 & 3.80 & 4.32 & 3.54 & 3.96 & 4.39 & 3.42 & 3.98 \\
\hline 6 & 3.55 & 3.10 & 3.31 & 3.36 & 2.88 & 3.17 & 4.13 & 3.17 & 3.69 & 4.14 & 3.52 & 3.89 \\
\hline 7 & 3.48 & 3.06 & 3.17 & 4.49 & 2.80 & 3.81 & 3.70 & 2.96 & 3.39 & 4.12 & 3.34 & 3.77 \\
\hline 8 & 3.23 & 2.56 & 2.91 & 4.52 & 4.08 & 4.31 & 3.40 & 2.79 & 3.16 & 4.05 & 3.20 & 3.69 \\
\hline 9 & 3.28 & 2.62 & 3.02 & 4.08 & 3.36 & 3.63 & 3.36 & 2.50 & 3.00 & 4.33 & 3.57 & 3.99 \\
\hline 10 & 3.56 & 2.54 & 3.03 & 3.97 & 2.96 & 3.53 & 3.57 & 2.60 & 3.13 & 4.64 & 3.87 & 4.23 \\
\hline 11 & 3.87 & 2.92 & 3.38 & 4.13 & 3.39 & 3.79 & 3.61 & 2.93 & 3.31 & 4.54 & 4.18 & 4.35 \\
\hline 12 & 3.59 & 2.64 & 2.94 & 4.44 & 3.43 & 3.96 & 3.71 & 2.97 & 3.37 & 4.42 & 3.53 & 4.12 \\
\hline 13 & 2.87 & 2.00 & 2.47 & 4.39 & 2.63 & 3.61 & 3.91 & 3.30 & 3.65 & 3.94 & 2.87 & 3.58 \\
\hline 14 & 3.53 & 2.26 & 2.81 & 2.63 & 1.19 & 1.66 & 4.32 & 3.35 & 3.87 & 3.64 & 2.87 & 3.36 \\
\hline 15 & 3.56 & 2.56 & 3.13 & 2.74 & 1.26 & 1.79 & 3.58 & 2.22 & 2.92 & 3.88 & 2.86 & 3.51 \\
\hline 16 & 3.63 & 2.85 & 3.30 & 3.32 & 2.49 & 2.92 & 3.19 & 2.15 & 2.81 & 4.19 & 3.03 & 3.79 \\
\hline 17 & 3.69 & 2.80 & 3.25 & 3.55 & 3.05 & 3.34 & 3.19 & 2.16 & 2.78 & 4.31 & 3.19 & 3.83 \\
\hline 18 & 3.59 & 2.78 & 3.24 & 3.85 & 3.32 & 3.55 & 2.89 & 1.94 & 2.51 & 4.33 & 3.28 & 3.82 \\
\hline 19 & 3.26 & 2.70 & 3.03 & 3.93 & 3.06 & 3.53 & 2.52 & 1.82 & 2.16 & 3.98 & 2.95 & 3.54 \\
\hline 20 & 4.28 & 2.92 & 3.61 & 3.56 & 2.59 & 3.15 & 2.55 & 1.60 & 2.16 & 3.71 & 2.78 & 3.33 \\
\hline 21 & 4.24 & 3.21 & 3.51 & 3.01 & 2.36 & 2.78 & 3.21 & 1.86 & 2.62 & 3.60 & 2.81 & 3.26 \\
\hline 22 & 3.99 & 2.99 & 3.59 & 3.24 & 2.34 & 2.92 & 3.95 & 2.71 & 3.36 & 3.34 & 2.78 & 3.07 \\
\hline 23 & 3.82 & 3.15 & 3.43 & 3.51 & 2.55 & 3.11 & 3.71 & 2.96 & 3.39 & 3.07 & 2.64 & 2.84 \\
\hline 24 & 3.41 & 2.37 & 2.92 & 3.43 & 2.49 & 3.03 & 3.53 & 2.92 & 3.26 & 2.80 & 2.43 & 2.66 \\
\hline 25 & 3.42 & 2.62 & 3.12 & 3.57 & 2.55 & 3.08 & 3.40 & 2.83 & 3.18 & 2.84 & 2.24 & 2.62 \\
\hline 26 & 3.56 & 2.54 & 3.12 & 3.91 & 2.90 & 3.47 & 3.64 & 3.30 & 3.48 & 3.00 & 2.25 & 2.69 \\
\hline 27 & 3.55 & 2.72 & 3.14 & 4.40 & 3.44 & 3.92 & 3.78 & 2.97 & 3.44 & 3.02 & 2.33 & 2.73 \\
\hline 28 & 3.24 & 2.38 & 2.84 & 4.57 & 3.77 & 4.15 & 3.44 & 2.93 & 3.26 & 3.08 & 2.32 & 2.76 \\
\hline 29 & --- & --- & -- & 3.88 & 3.14 & 3.53 & 3.57 & 2.90 & 3.33 & 3.15 & 2.30 & 2.84 \\
\hline 30 & --- & -- & -- & 3.83 & 3.40 & 3.61 & 4.40 & 2.88 & 3.94 & 3.34 & 2.38 & 2.93 \\
\hline 31 & --- & --- & --- & 3.82 & 3.23 & 3.53 & --- & --- & --- & 3.32 & 2.33 & 2.92 \\
\hline MONTH & 4.28 & 2.00 & 3.18 & 4.57 & 1.19 & 3.32 & 4.40 & 1.60 & 3.29 & 4.85 & 2.24 & 3.49 \\
\hline \multirow[t]{2}{*}{ DAY } & MAX & MIN & MEAN & MAX & MIN & MEAN & MAX & MIN & MEAN & MAX & MIN & MEAN \\
\hline & \multicolumn{3}{|c|}{ JUNE 1999} & \multicolumn{3}{|c|}{ JULY 1999} & \multicolumn{3}{|c|}{ AUGUST 1999} & & IBER & 99 \\
\hline 1 & 3.35 & 2.38 & 2.97 & 3.23 & 2.28 & 2.85 & 2.85 & 2.22 & 2.63 & 3.66 & 3.06 & 3.43 \\
\hline 2 & 3.69 & 2.62 & 3.24 & 3.64 & 2.46 & 3.17 & 2.93 & 2.40 & 2.72 & 3.84 & 3.14 & 3.59 \\
\hline 3 & 3.58 & 2.68 & 3.20 & 4.01 & 3.16 & 3.65 & 2.93 & 2.47 & 2.70 & 3.81 & 2.86 & 3.45 \\
\hline 4 & 3.45 & 2.60 & 3.09 & 3.74 & 3.16 & 3.44 & 2.90 & 2.38 & 2.70 & 3.82 & 2.64 & 3.36 \\
\hline 5 & 3.58 & 2.68 & 3.17 & 3.37 & 2.83 & 3.11 & 3.16 & 2.61 & 2.91 & 3.48 & 2.41 & 3.07 \\
\hline 6 & 3.48 & 2.82 & 3.16 & 3.22 & 2.74 & 2.94 & 3.39 & 2.49 & 3.07 & 3.39 & 2.47 & 3.05 \\
\hline 7 & 3.42 & 2.71 & 3.10 & 3.33 & 2.82 & 3.08 & 3.40 & 2.45 & 3.04 & 3.33 & 2.38 & 2.95 \\
\hline 8 & 3.61 & 3.06 & 3.33 & 3.54 & 3.00 & 3.31 & 3.48 & 2.24 & 3.02 & 3.27 & 2.38 & 2.97 \\
\hline 9 & 3.77 & 3.26 & 3.53 & 3.58 & 2.60 & 3.24 & 3.08 & 1.89 & 2.61 & 3.31 & 2.56 & 3.01 \\
\hline 10 & 3.74 & 2.86 & 3.42 & 3.67 & 2.69 & 3.33 & 2.77 & 1.84 & 2.37 & 3.82 & 2.72 & 3.38 \\
\hline 11 & 3.57 & 2.56 & 3.21 & 3.66 & 2.49 & 3.24 & 2.82 & 1.78 & 2.47 & 3.43 & 3.08 & 3.28 \\
\hline 12 & 3.54 & 2.58 & 3.21 & 3.54 & 2.47 & 3.08 & 2.81 & 2.07 & 2.50 & 3.46 & 3.08 & 3.28 \\
\hline 13 & 3.55 & 2.52 & 3.10 & 3.48 & 2.49 & 3.10 & 2.54 & 1.96 & 2.29 & 3.80 & 3.26 & 3.44 \\
\hline 14 & 3.48 & 2.44 & 3.02 & 3.31 & 2.38 & 2.97 & 2.43 & 1.88 & 2.23 & 3.99 & 3.42 & 3.73 \\
\hline 15 & 3.46 & 2.28 & 3.02 & 3.34 & 2.35 & 2.97 & 3.23 & 2.16 & 2.75 & 4.10 & 3.46 & 3.82 \\
\hline 16 & 3.62 & 2.54 & 3.20 & 3.49 & 2.71 & 3.19 & 3.36 & 3.01 & 3.17 & 4.06 & 3.29 & 3.73 \\
\hline 17 & 3.76 & 2.65 & 3.32 & 3.44 & 2.77 & 3.17 & 3.22 & 2.55 & 2.93 & 3.70 & 2.96 & 3.42 \\
\hline 18 & 4.18 & 3.18 & 3.75 & 3.55 & 3.01 & 3.31 & 3.05 & 2.45 & 2.78 & 3.61 & 2.89 & 3.32 \\
\hline 19 & 4.15 & 3.51 & 3.86 & 3.76 & 3.24 & 3.54 & 2.99 & 2.21 & 2.68 & 3.80 & 3.00 & 3.46 \\
\hline 20 & 4.12 & 3.54 & 3.84 & 3.76 & 3.13 & 3.55 & 3.05 & 2.25 & 2.71 & 3.74 & 3.16 & 3.49 \\
\hline 21 & 4.73 & 3.85 & 4.37 & 3.53 & 2.94 & 3.31 & 3.16 & 2.44 & 2.91 & 4.00 & 3.38 & 3.75 \\
\hline 22 & 3.85 & 3.10 & 3.58 & 3.42 & 2.54 & 3.12 & 3.84 & 2.69 & 3.42 & 4.39 & 3.42 & 4.06 \\
\hline 24 & 3.90 & 2.99 & 3.59 & 3.08 & 2.26 & 2.75 & 3.44 & 2.65 & 3.17 & 3.79 & 2.83 & 3.42 \\
\hline 25 & 3.55 & 2.62 & 3.28 & 3.24 & 2.26 & 2.85 & 3.39 & 2.41 & 3.06 & 3.83 & 2.78 & 3.37 \\
\hline 26 & 3.41 & 2.43 & 3.04 & 3.10 & 2.18 & 2.72 & 2.94 & 2.17 & 2.66 & 4.01 & 3.45 & 3.71 \\
\hline 27 & 3.38 & 2.38 & 2.97 & 3.08 & 2.14 & 2.66 & 2.73 & 2.06 & 2.52 & 4.07 & 3.33 & 3.72 \\
\hline 28 & 3.13 & 2.22 & 2.76 & 2.97 & 2.08 & 2.61 & 3.05 & 2.22 & 2.72 & 4.36 & 3.73 & 4.07 \\
\hline 29 & 3.03 & 2.09 & 2.64 & 2.90 & 2.07 & 2.59 & 3.08 & 2.53 & 2.85 & 4.40 & 3.45 & 3.95 \\
\hline 30 & 3.10 & 2.04 & 2.71 & 2.78 & 2.09 & 2.55 & 3.26 & 2.61 & 2.91 & 4.17 & 3.52 & 3.89 \\
\hline 31 & --- & --- & --- & 2.88 & 2.15 & 2.62 & 3.47 & 2.74 & 3.08 & --- & --- & --- \\
\hline MONTH & 4.73 & 2.04 & 3.27 & 4.01 & 2.07 & 3.06 & 3.84 & 1.78 & 2.81 & 4.40 & 2.38 & 3.50 \\
\hline
\end{tabular}


Table 2. Maximum, minimum, and mean gage heights in Christmas Bay, February 1999-February 2000—Continued

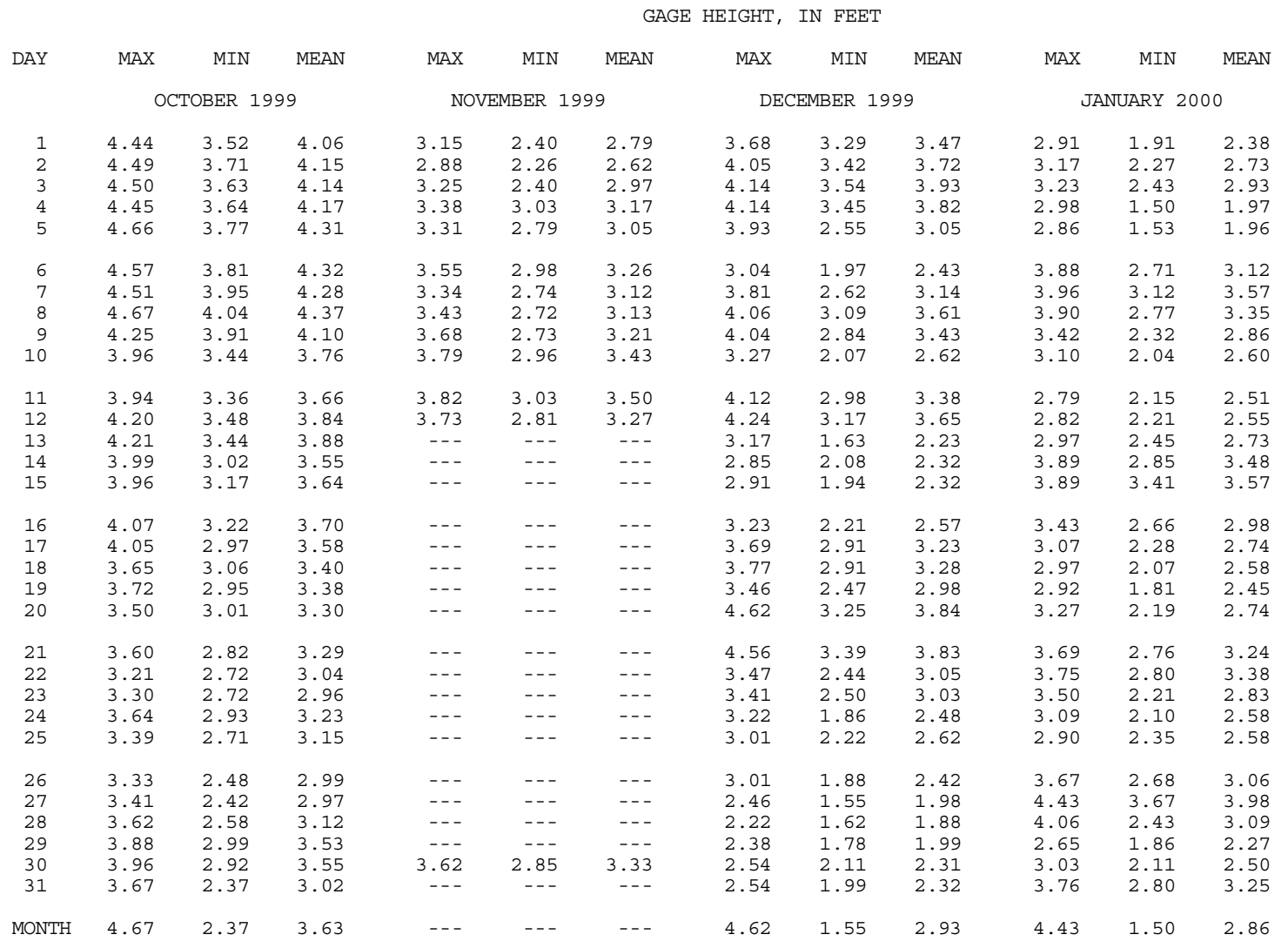

\begin{tabular}{|c|c|c|c|}
\hline \multirow[t]{2}{*}{ DAY } & MAX & MIN & MEAN \\
\hline & \multicolumn{3}{|c|}{ FEBRUARY 2000} \\
\hline 1 & 3.88 & 3.00 & 3.49 \\
\hline 2 & 3.79 & 2.94 & 3.33 \\
\hline 3 & 3.22 & 2.20 & 2.65 \\
\hline 4 & 2.60 & 1.95 & 2.30 \\
\hline 5 & 2.66 & 2.01 & 2.34 \\
\hline 6 & 2.77 & 2.08 & 2.50 \\
\hline 7 & 2.85 & 2.08 & 2.53 \\
\hline 8 & 2.47 & 1.93 & 2.26 \\
\hline 9 & 2.36 & 1.96 & 2.20 \\
\hline 10 & 2.82 & 2.20 & 2.49 \\
\hline 11 & 2.71 & 2.36 & 2.52 \\
\hline 12 & 2.90 & 2.26 & 2.61 \\
\hline 13 & 3.19 & 2.33 & 2.82 \\
\hline 14 & 3.25 & 2.50 & 2.92 \\
\hline 15 & 3.34 & 2.16 & 2.75 \\
\hline 16 & 3.38 & 2.43 & 3.00 \\
\hline 17 & 3.52 & 2.49 & 3.09 \\
\hline 18 & 3.51 & 2.53 & 3.10 \\
\hline 19 & 3.42 & 2.56 & 3.03 \\
\hline 20 & 3.30 & 2.69 & 3.05 \\
\hline 21 & 3.16 & 2.59 & 2.95 \\
\hline 22 & 3.29 & 2.83 & 3.10 \\
\hline 23 & 3.59 & 2.86 & 3.18 \\
\hline 24 & 3.29 & 2.61 & 2.90 \\
\hline 25 & 3.76 & 3.06 & 3.45 \\
\hline 26 & 3.66 & 3.09 & 3.40 \\
\hline 27 & 3.09 & 2.51 & 2.82 \\
\hline 28 & 3.60 & 2.40 & 2.99 \\
\hline 29 & 3.53 & 2.77 & 3.24 \\
\hline 30 & -- & -- & -- \\
\hline 31 & -- & --- & --- \\
\hline MONTH & 3.88 & 1.93 & 2.86 \\
\hline
\end{tabular}


Table 3. Maximum, minimum, and mean specific conductance, $\mathrm{pH}$, water temperature, and dissolved oxygen in Christmas Bay, February 1999-February 2000

SPECIFIC CONDUCTANCE, IN MICROSIEMENS PER CENTIMETER AT 25 DEGREES CELSIUS

\begin{tabular}{|c|c|c|c|c|c|c|c|c|c|c|c|c|}
\hline DAY & MAX & MIN & MEAN & MAX & MIN & MEAN & MAX & MIN & MEAN & MAX & MIN & MEAN \\
\hline & \multicolumn{3}{|c|}{ FEBRUARY 1999} & \multicolumn{3}{|c|}{ MARCH 1999} & \multicolumn{3}{|c|}{ APRIL 1999} & \multicolumn{3}{|c|}{ MAY 1999} \\
\hline 1 & 42500 & 41600 & 42100 & 43600 & 43100 & 43400 & 44300 & 43500 & 44000 & 49900 & 48600 & 49300 \\
\hline 2 & 42100 & 41800 & 42000 & 43700 & 42900 & 43300 & 44600 & 43500 & 44200 & 49100 & 47000 & 48000 \\
\hline 3 & 42800 & 42100 & 42400 & 43700 & 43300 & 43500 & 44300 & 43700 & 44000 & 48100 & 46000 & 47300 \\
\hline 4 & 43200 & 42700 & 43000 & 43800 & 43200 & 43500 & 44000 & 41200 & 42500 & 47400 & 43800 & 46200 \\
\hline 5 & 43400 & 43200 & 43300 & 45900 & 43300 & 44000 & 43400 & 41900 & 42900 & 47000 & 44700 & 46100 \\
\hline 6 & 43500 & 42800 & 43100 & 45500 & 43900 & 44700 & 43300 & 40800 & 41800 & 46900 & 43100 & 45500 \\
\hline 7 & 43900 & 42800 & 43400 & 45900 & 43900 & 44800 & 43100 & 42100 & 42700 & 47100 & 44600 & 45700 \\
\hline 8 & 44500 & 43900 & 44200 & 45900 & 45200 & 45600 & 43100 & 42100 & 42400 & 47400 & 45100 & 46000 \\
\hline 9 & 44900 & 44200 & 44600 & 45700 & 44600 & 45000 & 42500 & 41800 & 42200 & 46000 & 40600 & 44200 \\
\hline 10 & 44900 & 44100 & 44600 & 45200 & 44800 & 45000 & 42300 & 41400 & 41800 & 44800 & 38400 & 42000 \\
\hline 11 & 45000 & 43700 & 44300 & 45000 & 43500 & 44200 & 41900 & 40600 & 41200 & 44300 & 39900 & 42700 \\
\hline 12 & 45600 & 44800 & 45200 & 43800 & 43300 & 43500 & 41400 & 40600 & 41000 & 44400 & 40200 & 42800 \\
\hline 13 & 46300 & 43700 & 44700 & 43900 & 42300 & 42900 & 41100 & 40200 & 40700 & 44000 & 40900 & 42300 \\
\hline 14 & 46300 & 45100 & 45600 & 45900 & 43100 & 45000 & 41700 & 40500 & 40900 & 43100 & 39600 & 41900 \\
\hline 15 & 46700 & 46000 & 46300 & 45900 & 44700 & 45500 & 42300 & 39600 & 41500 & 43700 & 40700 & 42300 \\
\hline 16 & 46700 & 46100 & 46500 & 47400 & 44500 & 45800 & 42300 & 40400 & 41800 & 43000 & 39500 & 41600 \\
\hline 17 & 46600 & 45400 & 45700 & 48500 & 47000 & 47900 & 42100 & 40600 & 41600 & 43100 & 40000 & 41700 \\
\hline 18 & 45600 & 44700 & 45300 & 49600 & 48400 & 49100 & 42100 & 40100 & 41800 & 42700 & 37900 & 40400 \\
\hline 19 & 44700 & 42300 & 43200 & 49100 & 48500 & 48900 & 42200 & 41700 & 42000 & 44100 & 38900 & 42000 \\
\hline 20 & 42800 & 41200 & 42300 & 48900 & 48000 & 48600 & 42400 & 39100 & 41800 & 44700 & 41400 & 44000 \\
\hline 21 & 44400 & 42000 & 43400 & 48500 & 48000 & 48100 & 45800 & 42200 & 43500 & 44300 & 41400 & 43300 \\
\hline 22 & 45500 & 44000 & 44900 & 48300 & 47900 & 48100 & 50400 & 44300 & 47500 & 44200 & 42300 & 43600 \\
\hline 23 & 45900 & 44600 & 45400 & 48500 & 48200 & 48300 & 47500 & 45800 & 46400 & 43400 & 38900 & 42400 \\
\hline 24 & 45800 & 42700 & 44700 & 48300 & 47300 & 47800 & 46800 & 44300 & 45400 & 43500 & 39400 & 42800 \\
\hline 25 & 44400 & 40000 & 43200 & 47700 & 46400 & 47000 & 49400 & 45500 & 47100 & 43200 & 40400 & 42000 \\
\hline 26 & 42800 & 42100 & 42500 & 47600 & 46300 & 46900 & 47600 & 45900 & 46800 & 43500 & 38500 & 42200 \\
\hline 27 & 43000 & 42400 & 42700 & 47600 & 46700 & 47400 & 48700 & 46100 & 47700 & 43500 & 41800 & 42900 \\
\hline 28 & 43500 & 43000 & 43200 & 47300 & 45000 & 46000 & 47500 & 46200 & 47200 & 44400 & 41300 & 43200 \\
\hline 29 & -- & --- & --- & 46900 & 43500 & 44800 & 47200 & 45800 & 46900 & 44400 & 40500 & 43200 \\
\hline 30 & --- & --- & --- & 43800 & 43500 & 43700 & 49900 & 46100 & 48400 & 45900 & 42000 & 43500 \\
\hline 31 & --- & --- & --- & 44400 & 43400 & 43900 & --- & --- & --- & 46000 & 41000 & 43700 \\
\hline MONTH & 46700 & 40000 & 44000 & 49600 & 42300 & 45700 & 50400 & 39100 & 43700 & 49900 & 37900 & 43700 \\
\hline \multirow[t]{2}{*}{ DAY } & MAX & MIN & MEAN & MAX & MIN & MEAN & MAX & MIN & MEAN & MAX & MIN & MEAN \\
\hline & \multicolumn{3}{|c|}{ JUNE 1999} & \multicolumn{3}{|c|}{ JULY 1999} & \multicolumn{3}{|c|}{ AUGUST 1999} & \multicolumn{3}{|c|}{ SEPTEMBER 1999} \\
\hline 1 & 45900 & 41300 & 43500 & 42100 & 40300 & 41300 & 49300 & 47900 & 48500 & 55500 & 54300 & 55200 \\
\hline 2 & 47100 & 41900 & 44100 & 44100 & 40400 & 42500 & 49400 & 48000 & 48700 & 55700 & 54100 & 55300 \\
\hline 3 & 46600 & 42600 & 44200 & 46700 & 41500 & 44400 & 49900 & 48600 & 49400 & 55500 & 54500 & 55100 \\
\hline 4 & 46900 & 42900 & 44500 & 45800 & 43900 & 44700 & 50000 & 48600 & 49600 & 55800 & 54000 & 55000 \\
\hline 5 & 45600 & 42800 & 44200 & 45200 & 44100 & 44700 & 50000 & 49200 & 49700 & 55900 & 54000 & 55100 \\
\hline 6 & 46700 & 42100 & 44600 & 45500 & 44200 & 44800 & 50000 & 49400 & 49800 & 55800 & 54200 & 55000 \\
\hline 7 & 46900 & 42900 & 45600 & 46100 & 44700 & 45400 & 50100 & 49700 & 49900 & 56000 & 54500 & 55000 \\
\hline 8 & 47000 & 43600 & 45400 & 46700 & 45500 & 46200 & 50500 & 49800 & 50100 & 56000 & 54500 & 55000 \\
\hline 9 & 47200 & 42800 & 45700 & 45800 & 45300 & 45500 & 51000 & 50000 & 50800 & 55400 & 53900 & 55000 \\
\hline 10 & 47200 & 41700 & 46000 & 45400 & 44400 & 45000 & 51000 & 50000 & 50700 & 55700 & 54200 & 54900 \\
\hline 11 & 47300 & 39500 & 45900 & 44800 & 44100 & 44400 & 52200 & 50700 & 51400 & 55400 & 54900 & 55100 \\
\hline 12 & 47000 & 41800 & 45800 & 44600 & 44200 & 44300 & 52400 & 51300 & 51800 & 55700 & 55100 & 55400 \\
\hline 13 & 46900 & 41900 & 45000 & 44700 & 44300 & 44600 & 53600 & 51800 & 52800 & 55600 & 55100 & 55400 \\
\hline 14 & 45900 & 39300 & 43000 & 44800 & 44200 & 44600 & 53800 & 51400 & 53400 & 55900 & 54800 & 55400 \\
\hline 15 & 44800 & 42500 & 43600 & 44800 & 43700 & 44200 & 53800 & 53300 & 53500 & 55900 & 55300 & 55600 \\
\hline 16 & 45200 & 40700 & 43500 & 44800 & 44000 & 44500 & 53400 & 52700 & 53300 & 56500 & 55300 & 55800 \\
\hline 17 & 46800 & 42000 & 45300 & 45800 & 44600 & 44900 & 53500 & 52600 & 53300 & 56300 & 55500 & 55900 \\
\hline 18 & 46900 & 42800 & 44600 & 45900 & 45100 & 45600 & 53800 & 53100 & 53500 & 56400 & 55600 & 56000 \\
\hline 19 & 46600 & 40800 & 44000 & 46200 & 45700 & 45900 & 54300 & 53200 & 53900 & 56500 & 55100 & 56100 \\
\hline 20 & 42200 & 38500 & 40600 & 46900 & 45600 & 46500 & 54400 & 53100 & 54300 & 56700 & 55700 & 56200 \\
\hline 21 & 40400 & 38200 & 39400 & 46800 & 45400 & 46000 & 55300 & 53900 & 54800 & 56400 & 54700 & 56000 \\
\hline 22 & 40200 & 37000 & 39100 & 46500 & 45400 & 45800 & 56400 & 54800 & 55900 & 56800 & 55500 & 56200 \\
\hline 23 & 40000 & 37800 & 39300 & 45900 & 45400 & 45700 & 56500 & 55200 & 55900 & 56600 & 55100 & 56300 \\
\hline 24 & 40000 & 37800 & 38900 & 45900 & 45400 & 45700 & 56000 & 55400 & 55700 & 56900 & 55800 & 56300 \\
\hline 25 & 40200 & 38800 & 39500 & 46100 & 44700 & 45700 & 56400 & 54300 & 55500 & 57100 & 53700 & 56200 \\
\hline 26 & 39800 & 38900 & 39400 & 45800 & 44700 & 45600 & 56700 & 55200 & 55800 & 56100 & 55000 & 55600 \\
\hline 27 & 39900 & 38100 & 38900 & 46200 & 45400 & 45700 & 57000 & 55500 & 56000 & --- & --- & --- \\
\hline 28 & 39600 & 38300 & 39100 & 45900 & 45300 & 45500 & 56600 & 54700 & 55700 & --- & --- & --- \\
\hline 29 & 40200 & 39000 & 39500 & 46200 & 45400 & 45800 & 55900 & 54700 & 55500 & -- & --- & --- \\
\hline 30 & 41800 & 39600 & 40800 & 46900 & 45700 & 46200 & 55700 & 54400 & 55200 & --- & --- & --- \\
\hline 31 & --- & --- & --- & 47900 & 46600 & 46900 & 55700 & 53200 & 55300 & --- & --- & --- \\
\hline MONTH & 47300 & 37000 & 42800 & 47900 & 40300 & 45100 & 57000 & 47900 & 52900 & --- & --- & --- \\
\hline
\end{tabular}


Table 3. Maximum, minimum, and mean specific conductance, $\mathrm{pH}$, water temperature, and dissolved oxygen in Christmas Bay, February 1999-February 2000-Continued

SPECIFIC CONDUCTANCE, IN MICROSIEMENS PER CENTIMETER AT 25 DEGREES CELSIUS

\begin{tabular}{|c|c|c|c|c|c|c|c|c|c|c|c|c|}
\hline \multirow[t]{2}{*}{ DAY } & MAX & MIN & MEAN & MAX & MIN & MEAN & MAX & MIN & MEAN & MAX & MIN & MEAN \\
\hline & \multicolumn{3}{|c|}{ OCTOBER 1999} & \multicolumn{3}{|c|}{ NOVEMBER 1999} & \multicolumn{3}{|c|}{ DECEMBER 1999} & \multicolumn{3}{|c|}{ JANUARY 2000} \\
\hline 1 & --- & -- & -- & 53000 & 51000 & 51900 & 54300 & 45600 & 53300 & 48900 & 47800 & 48600 \\
\hline 2 & --- & -- & -- & 53800 & 52000 & 52600 & 54200 & 50500 & 53300 & 49000 & 43600 & 48600 \\
\hline 3 & --- & -- & -- & 53400 & 51800 & 52400 & 53000 & 48200 & 52100 & 49600 & 47200 & 48700 \\
\hline 4 & --- & -- & -- & 52300 & 51600 & 52000 & 52200 & 49200 & 51700 & 49400 & 46700 & 48800 \\
\hline 5 & --- & --- & --- & 52600 & 50900 & 52000 & 50600 & 50000 & 50300 & 49900 & 48700 & 49200 \\
\hline 6 & --- & -- & -- & 52200 & 50000 & 51600 & 50900 & 40700 & 50300 & 52700 & 49100 & 49900 \\
\hline 7 & -- & -- & -- & 53100 & 47000 & 50600 & 50600 & 48500 & 49900 & 52900 & 52000 & 52500 \\
\hline 8 & --- & -- & -- & 52700 & 50200 & 51700 & 50000 & 46500 & 49400 & 52400 & 50800 & 51600 \\
\hline 9 & --- & -- & --- & 52300 & 50900 & 51600 & 50300 & 49100 & 49900 & 51600 & 49900 & 51100 \\
\hline 10 & --- & -- & --- & 52100 & 50200 & 51400 & 49800 & 46100 & 48700 & 50900 & 49700 & 50300 \\
\hline 11 & --- & -- & --- & 52000 & 46600 & 51100 & 50200 & 48700 & 49800 & 51200 & 49900 & 50500 \\
\hline 12 & --- & -- & -- & 52300 & 49300 & 51400 & 50200 & 48000 & 49500 & 51300 & 49900 & 50500 \\
\hline 13 & --- & -- & -- & -- & --- & -- & 49400 & 47200 & 49000 & 51600 & 49800 & 50700 \\
\hline 14 & 50900 & 50300 & 50500 & $-\ldots$ & -- & $-\ldots$ & 49500 & 36700 & 47000 & 50900 & 50400 & 50600 \\
\hline 15 & 50900 & 50200 & 50500 & --- & --- & -- & 49500 & 38400 & 47000 & 51100 & 50600 & 50900 \\
\hline 16 & 51200 & 50500 & 50800 & --- & -- & --- & 49800 & 37200 & 46900 & 50900 & 50100 & 50600 \\
\hline 17 & 51000 & 50400 & 50700 & --- & -- & -- & 50100 & 31300 & 45400 & 51000 & 49500 & 50400 \\
\hline 18 & 51300 & 50400 & 50800 & -- & --- & -- & 49300 & 41100 & 47700 & 51100 & 49900 & 50600 \\
\hline 19 & 51300 & 50300 & 50700 & -- & --- & -- & 49200 & 36400 & 45700 & 51000 & 50100 & 50600 \\
\hline 20 & 51600 & 50800 & 51300 & -- & --- & -- & 49200 & 47600 & 48400 & 50900 & 50300 & 50600 \\
\hline 21 & 51900 & 50600 & 51300 & -- & -- & -- & 47900 & 45100 & 47600 & 51000 & 49100 & 50500 \\
\hline 22 & 52100 & 50300 & 51600 & -- & --- & -- & 48400 & 47500 & 47900 & 50900 & 49700 & 50400 \\
\hline 23 & 52100 & 51100 & 51700 & -- & -- & -- & 48600 & 47500 & 48200 & 50800 & 47500 & 50100 \\
\hline 24 & 51800 & 50300 & 51300 & -- & -- & -- & 48400 & 44300 & 47700 & 50400 & 50000 & 50200 \\
\hline 25 & 52200 & 51100 & 51600 & --- & --- & --- & 48100 & 44000 & 47700 & 50400 & 48100 & 50000 \\
\hline 26 & 52300 & 50700 & 51500 & -- & -- & -- & 48200 & 39500 & 45800 & 50400 & 49700 & 50000 \\
\hline 27 & 52300 & 49800 & 51300 & -- & -- & -- & 48600 & 42700 & 47500 & 50400 & 47400 & 49500 \\
\hline 28 & 51900 & 49900 & 51100 & -- & -- & -- & 48800 & 34400 & 45700 & 48000 & 47100 & 47700 \\
\hline 29 & 51200 & 50200 & 50500 & -- & --- & -- & 48700 & 44000 & 47500 & 47500 & 45800 & 46400 \\
\hline 30 & 51600 & 47800 & 50300 & 54900 & 52400 & 54500 & 49100 & 42000 & 48200 & 47300 & 46200 & 46600 \\
\hline 31 & 52700 & 48100 & 51100 & -- & --- & -- & 49000 & 47100 & 48600 & 47500 & 46900 & 47100 \\
\hline MONTH & --- & -- & --- & --- & -- & -- & 54300 & 31300 & 48600 & 52900 & 43600 & 49800 \\
\hline
\end{tabular}

DAY MAX MIN MEAN

FEBRUARY 2000

$\begin{array}{rrrr}1 & 47800 & 45800 & 47000 \\ 2 & 46900 & 45500 & 46300 \\ 3 & 46700 & 45500 & 45900 \\ 4 & 45800 & 45100 & 45400 \\ 5 & 45200 & 44300 & 44700 \\ & & & \\ 6 & 45600 & 44800 & 45200 \\ 7 & 45700 & 42900 & 44800 \\ 8 & 44400 & 40700 & 43700 \\ 9 & 44300 & 42600 & 43700 \\ 10 & 44100 & 42600 & 43800 \\ & & & \\ 11 & 43900 & 43200 & 43600 \\ 12 & 43600 & 39000 & 43200 \\ 13 & 43400 & 40400 & 43100 \\ 14 & 43200 & 39900 & 42400 \\ 15 & 43400 & 37500 & 42800 \\ & & & \\ 16 & 43200 & 36500 & 42500 \\ 17 & 43400 & 36500 & 42700 \\ 18 & 43800 & 39500 & 42700 \\ 19 & 43200 & 42000 & 42800 \\ 20 & 43600 & 40900 & 43100 \\ & & & \\ 21 & 43700 & 37700 & 43200 \\ 22 & 43700 & 37100 & 43000 \\ 23 & 44000 & 36500 & 43200 \\ 24 & 43800 & 43000 & 43500 \\ 25 & 44500 & 43200 & 43800 \\ 26 & 44300 & 43500 & 43900 \\ 27 & 44400 & 43800 & 44100 \\ 28 & 44500 & 41800 & 44000 \\ 29 & 50400 & 43600 & 47500 \\ 30 & --- & --- & --- \\ 31 & --- & --- & --- \\ & & & \\ \text { MONTH } & 50400 & 36500 & 44000\end{array}$


Table 3. Maximum, minimum, and mean specific conductance, $\mathrm{pH}$, water temperature, and dissolved oxygen in Christmas Bay, February 1999-February 2000-Continued

PH, IN STANDARD UNITS

\begin{tabular}{|c|c|c|c|c|c|c|c|c|c|c|c|c|}
\hline DAY & MAX & MIN & MEAN & MAX & MIN & MEAN & MAX & MIN & MEAN & MAX & MIN & MEAN \\
\hline & \multicolumn{3}{|c|}{ FEBRUARY 1999} & \multicolumn{3}{|c|}{ MARCH 1999} & \multicolumn{3}{|c|}{ APRIL 1999} & \multicolumn{3}{|c|}{ MAY 1999} \\
\hline 1 & 8.3 & 8.2 & 8.2 & 8.3 & 8.1 & 8.2 & 8.2 & 8.0 & 8.1 & 8.2 & 8.1 & 8.2 \\
\hline 2 & 8.3 & 8.2 & 8.2 & 8.2 & 8.1 & 8.2 & 8.1 & 8.0 & 8.0 & 8.2 & 8.1 & 8.2 \\
\hline 3 & 8.3 & 8.2 & 8.2 & 8.2 & 8.1 & 8.2 & 8.1 & 8.0 & 8.0 & 8.2 & 8.1 & 8.1 \\
\hline 4 & 8.2 & 8.2 & 8.2 & 8.2 & 8.1 & 8.2 & 8.1 & 8.0 & 8.0 & 8.2 & 8.1 & 8.1 \\
\hline 5 & 8.2 & 8.2 & 8.2 & 8.3 & 8.1 & 8.1 & 8.1 & 7.9 & 8.0 & 8.1 & 7.9 & 8.1 \\
\hline 6 & 8.2 & 8.1 & 8.2 & 8.3 & 8.1 & 8.2 & 8.2 & 8.0 & 8.1 & 8.2 & 7.9 & 8.1 \\
\hline 7 & 8.3 & 8.1 & 8.2 & 8.2 & 8.1 & 8.2 & 8.0 & 7.9 & 8.0 & 8.2 & 8.1 & 8.1 \\
\hline 8 & 8.3 & 8.1 & 8.3 & 8.2 & 8.1 & 8.1 & 8.0 & 7.9 & 8.0 & 8.1 & 8.0 & 8.1 \\
\hline 9 & 8.3 & 8.2 & 8.2 & 8.3 & 8.1 & 8.2 & 8.1 & 7.9 & 8.0 & 8.2 & 8.0 & 8.1 \\
\hline 10 & 8.2 & 8.2 & 8.2 & 8.2 & 8.1 & 8.1 & 8.1 & 8.0 & 8.1 & 8.2 & 8.0 & 8.2 \\
\hline 11 & 8.3 & 8.2 & 8.2 & -- & -- & -- & 8.3 & 8.1 & 8.2 & 8.2 & 8.1 & 8.1 \\
\hline 12 & 8.3 & 8.2 & 8.2 & -- & - - - &.-- & 8.2 & 8.1 & 8.1 &.- & --- &.- \\
\hline 13 & 8.3 & 8.2 & 8.3 & -- & -- & -- & 8.1 & 8.1 & 8.1 & - - - & --- & -- \\
\hline 14 & 8.3 & 8.3 & 8.3 & -- & - - - & -- & 8.1 & 8.0 & 8.1 & - - - & --- &.- \\
\hline 15 & 8.3 & 8.2 & 8.3 & --- & --- & --- & 8.1 & 8.0 & 8.1 & --- & --- & --- \\
\hline 16 & 8.3 & 8.2 & 8.2 & --- & - - - & -- & 8.3 & 8.0 & 8.1 & - - - & --- &.-- \\
\hline 17 & 8.2 & 8.2 & 8.2 & -- & -- & -- & 8.4 & 8.2 & 8.3 & -- & -- & -- \\
\hline 18 & 8.3 & 8.2 & 8.2 & 8.2 & 8.1 & 8.1 & 8.3 & 8.2 & 8.2 & - - - &.-- &.- \\
\hline 19 & 8.3 & 8.2 & 8.2 & 8.4 & 8.0 & 8.1 & 8.2 & 8.1 & 8.1 & -- & -- & -- \\
\hline 20 & 8.3 & 8.2 & 8.2 & 8.2 & 8.0 & 8.1 & 8.1 & 8.1 & 8.1 & -- & --- & --- \\
\hline 21 & 8.3 & 8.2 & 8.3 & 8.1 & 8.0 & 8.0 & 8.1 & 8.0 & 8.1 & -- & -- & -- \\
\hline 22 & 8.3 & 8.2 & 8.3 & 8.1 & 7.9 & 8.0 & 8.1 & 8.0 & 8.0 & - - - &.-- &.- \\
\hline 23 & 8.3 & 8.1 & 8.2 & 8.1 & 7.9 & 7.9 & 8.1 & 7.9 & 8.0 & -- & -- & -- \\
\hline 24 & 8.3 & 8.2 & 8.2 & 8.1 & 7.9 & 8.0 & 8.1 & 8.0 & 8.1 & -- & -- & -- \\
\hline 25 & 8.3 & 8.1 & 8.1 & 8.0 & 7.9 & 8.0 & 8.2 & 8.0 & 8.1 & --- & --- & --- \\
\hline 26 & 8.3 & 8.1 & 8.2 & 8.0 & 7.9 & 7.9 & 8.1 & 7.8 & 8.0 & - - - & -.- &.-- \\
\hline 27 & 8.2 & 8.1 & 8.1 & 8.0 & 7.9 & 8.0 & 8.1 & 7.8 & 7.9 & --- & -- & -- \\
\hline 28 & 8.2 & 8.1 & 8.1 & 8.1 & 7.9 & 8.0 & 8.1 & 7.9 & 8.0 & -- & -- & -- \\
\hline 29 &.- & -- & -- & 8.2 & 7.9 & 8.1 & 8.1 & 7.9 & 8.1 & - - - & --- &.- \\
\hline 30 & -- & -- & -- & 8.2 & 8.0 & 8.1 & 8.2 & 8.1 & 8.1 & -- & -- & -- \\
\hline 31 & -- & --- & --- & 8.2 & 8.0 & 8.1 & --- & --- & --- & -- & --- & --- \\
\hline MONTH & 8.3 & 8.1 & 8.2 & --- & -- & -- & 8.4 & 7.8 & 8.1 & -- & --- & -- \\
\hline \multirow[t]{2}{*}{ DAY } & MAX & MIN & MEAN & MAX & MIN & MEAN & MAX & MIN & MEAN & MAX & MIN & MEAN \\
\hline & \multicolumn{3}{|c|}{ JUNE 1999} & \multicolumn{3}{|c|}{ JULY 1999} & \multicolumn{3}{|c|}{ AUGUST 1999} & \multicolumn{3}{|c|}{ SEPTEMBER 1999} \\
\hline 1 & -- & --- & -- & 8.4 & 8.3 & 8.3 & 8.3 & 8.1 & 8.2 & 8.0 & 7.8 & 7.9 \\
\hline 2 & - - - & --- & - - - & 8.4 & 8.3 & 8.4 & 8.4 & 8.0 & 8.1 & 8.0 & 7.9 & 8.0 \\
\hline 3 & -- & -- & -- & 8.6 & 8.3 & 8.4 & 8.5 & 8.0 & 8.2 & 8.1 & 8.0 & 8.0 \\
\hline 4 & -- & --- & -- & 8.5 & 8.2 & 8.4 & 8.6 & 8.0 & 8.2 & 8.1 & 8.0 & 8.0 \\
\hline 5 & --- & --- & --- & 8.7 & 8.1 & 8.4 & 8.3 & 8.0 & 8.1 & 8.1 & 8.0 & 8.0 \\
\hline 6 & - - - & - - - & - - - & 8.9 & 8.2 & 8.5 & 8.4 & 7.9 & 8.1 & 8.1 & 8.0 & 8.0 \\
\hline 7 & -- & -- & -- & 8.8 & 8.1 & 8.3 & 8.4 & 8.0 & 8.1 & 8.1 & 8.0 & 8.0 \\
\hline 8 & -- & -- & -- & 8.6 & 8.1 & 8.3 & 8.3 & 8.0 & 8.2 & 8.1 & 8.0 & 8.0 \\
\hline 9 & - - - & - - - & -. - & 8.4 & 8.1 & 8.2 & 8.2 & 8.0 & 8.1 & 8.1 & 8.0 & 8.0 \\
\hline 10 & --- & --- & --- & 8.4 & 8.1 & 8.3 & 8.2 & 8.1 & 8.1 & 8.1 & 8.0 & 8.0 \\
\hline 11 & -- & --- & --- & 8.5 & 8.2 & 8.3 & 8.2 & 8.1 & 8.1 & 8.1 & 8.0 & 8.0 \\
\hline 12 & -- & -- & -- & 8.4 & 8.1 & 8.3 & 8.3 & 8.1 & 8.1 & 8.1 & 8.0 & 8.0 \\
\hline 13 & -- & -- & -- & 8.4 & 8.1 & 8.2 & 8.2 & 8.1 & 8.1 & 8.1 & 8.0 & 8.0 \\
\hline 14 & -- & -- & -- & 8.6 & 8.2 & 8.4 & 8.2 & 7.9 & 8.1 & 8.1 & 8.0 & 8.0 \\
\hline 15 & -- & --- & --- & 8.5 & 8.1 & 8.3 & 8.1 & 7.9 & 8.1 & 8.1 & 8.0 & 8.1 \\
\hline 16 & - - - & --- & -- & 8.4 & 8.1 & 8.3 & 8.2 & 7.9 & 8.1 & 8.1 & 8.0 & 8.1 \\
\hline 17 & --- & --- & -- & 8.5 & 8.2 & 8.4 & 8.3 & 7.8 & 8.0 & 8.1 & 8.0 & 8.1 \\
\hline 18 & -- & -- & -- & 8.5 & 8.2 & 8.3 & 8.2 & 7.8 & 8.0 & 8.1 & 8.0 & 8.1 \\
\hline 19 & - - - & - - - & - - - & 8.4 & 8.1 & 8.3 & 8.2 & 7.8 & 8.0 & 8.1 & 8.0 & 8.1 \\
\hline 20 & --- & --- & --- & 8.3 & 8.1 & 8.2 & 8.2 & 7.9 & 8.0 & 8.1 & 8.1 & 8.1 \\
\hline 21 & -- & --- & -- & 8.4 & 8.1 & 8.2 & 8.2 & 7.8 & 8.0 & 8.1 & 8.1 & 8.1 \\
\hline 22 & --- & -- & -- & 8.5 & 8.1 & 8.3 & 8.1 & 8.0 & 8.1 & 8.1 & 8.1 & 8.1 \\
\hline 23 & -- & -- & -- & 8.5 & 8.1 & 8.2 & 8.2 & 7.9 & 8.1 & 8.2 & 8.1 & 8.2 \\
\hline 24 & -- & -- & -- & 8.3 & 8.1 & 8.2 & 8.1 & 7.8 & 7.9 & 8.2 & 8.2 & 8.2 \\
\hline 25 & -- & --- & --- & 8.4 & 8.1 & 8.2 & 7.8 & 7.8 & 7.8 & 8.2 & 8.1 & 8.2 \\
\hline 26 & 8.4 & 8.3 & 8.3 & 8.3 & 8.2 & 8.2 & 7.9 & 7.7 & 7.8 & 8.2 & 8.1 & 8.2 \\
\hline 27 & 8.4 & 8.3 & 8.3 & 8.3 & 8.0 & 8.2 & 7.9 & 7.8 & 7.8 & - - - & - - - & - - \\
\hline 28 & 8.4 & 8.3 & 8.3 & 8.3 & 8.1 & 8.2 & 7.9 & 7.7 & 7.8 & -- & -- & -- \\
\hline 29 & 8.5 & 8.3 & 8.4 & 8.4 & 7.9 & 8.2 & 7.9 & 7.7 & 7.8 & - - - & - - - &.- \\
\hline 30 & 8.4 & 8.3 & 8.4 & 8.4 & 8.1 & 8.2 & 7.9 & 7.8 & 7.8 & -- & -- & -- \\
\hline 31 & --- & --- & --- & 8.3 & 8.1 & 8.2 & 7.9 & 7.8 & 7.9 & -- & --- & --- \\
\hline MONTH & --- & --- & -.- & 8.9 & 7.9 & 8.3 & 8.6 & 7.7 & 8.0 & - - - & - - - & --- \\
\hline
\end{tabular}


Table 3. Maximum, minimum, and mean specific conductance, $\mathrm{pH}$, water temperature, and dissolved oxygen in Christmas Bay, February 1999-February 2000-Continued

PH, IN STANDARD UNITS

\begin{tabular}{|c|c|c|c|c|c|c|c|c|c|c|c|c|}
\hline \multirow[t]{2}{*}{ DAY } & MAX & MIN & MEAN & MAX & MIN & MEAN & MAX & MIN & MEAN & MAX & MIN & MEAN \\
\hline & \multicolumn{3}{|c|}{ OCTOBER 1999} & \multicolumn{3}{|c|}{ NOVEMBER 1999} & \multicolumn{3}{|c|}{ DECEMBER 1999} & \multicolumn{3}{|c|}{ JANUARY 2000} \\
\hline 1 & --- & - - - &.-- & 8.2 & 8.2 & 8.2 & 8.3 & 8.2 & 8.3 & 8.3 & 8.1 & 8.2 \\
\hline 2 & -- & -- & --- & 8.2 & 8.2 & 8.2 & 8.3 & 8.2 & 8.2 & 8.3 & 8.2 & 8.2 \\
\hline 3 & --- & -- & --- & 8.3 & 8.2 & 8.2 & 8.2 & 8.2 & 8.2 & 8.3 & 8.1 & 8.2 \\
\hline 4 & -- & --- & -- & 8.2 & 8.2 & 8.2 & 8.2 & 8.1 & 8.2 & 8.3 & 8.2 & 8.3 \\
\hline 5 & --- & --- & --- & 8.2 & 8.2 & 8.2 & 8.2 & 8.2 & 8.2 & 8.3 & 8.2 & 8.2 \\
\hline 6 & --- & $-\ldots$ &.-- & 8.2 & 8.1 & 8.2 & 8.3 & 8.2 & 8.2 & 8.3 & 8.2 & 8.2 \\
\hline 7 & -- & -- & --- & 8.2 & 8.1 & 8.2 & 8.3 & 8.2 & 8.2 & 8.2 & 8.2 & 8.2 \\
\hline 8 & --- & --- & --- & 8.3 & 8.1 & 8.2 & 8.3 & 8.2 & 8.2 & 8.2 & 7.9 & 8.2 \\
\hline 9 & --- &.-- & --- & 8.2 & 8.1 & 8.2 & 8.3 & 8.2 & 8.2 & 8.2 & 7.9 & 8.1 \\
\hline 10 & --- & --- & --- & 8.2 & 8.1 & 8.2 & 8.3 & 8.2 & 8.3 & 8.2 & 7.9 & 8.2 \\
\hline 11 & --- & --- & --- & 8.2 & 8.1 & 8.2 & 8.3 & 8.3 & 8.3 & 8.2 & 8.0 & 8.1 \\
\hline 12 & --- & -- & --- & 8.3 & 8.2 & 8.2 & 8.3 & 8.3 & 8.3 & 8.2 & 8.1 & 8.2 \\
\hline 13 & --- & -- & --- & -- & -- & -- & 8.3 & 8.2 & 8.2 & 8.3 & 8.0 & 8.1 \\
\hline 14 & 8.2 & 8.2 & 8.2 & -.. & -. - & --- & 8.2 & 8.1 & 8.2 & 8.3 & 8.2 & 8.3 \\
\hline 15 & 8.2 & 8.2 & 8.2 & --- & --- & --- & 8.2 & 8.2 & 8.2 & 8.3 & 8.2 & 8.2 \\
\hline 16 & 8.2 & 8.2 & 8.2 & -- & -- & -- & 8.3 & 8.1 & 8.2 & 8.3 & 8.1 & 8.2 \\
\hline 17 & 8.2 & 8.1 & 8.2 &.- & 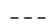 & --- & 8.3 & 8.1 & 8.2 & 8.2 & 8.1 & 8.2 \\
\hline 18 & 8.2 & 8.2 & 8.2 &.- & --- & -- & 8.3 & 8.2 & 8.3 & 8.3 & 8.1 & 8.2 \\
\hline 19 & 8.2 & 8.2 & 8.2 &.- & --- & --- & 8.3 & 8.2 & 8.2 & 8.2 & 8.2 & 8.2 \\
\hline 20 & 8.2 & 8.1 & 8.2 & --- & --- & --- & 8.3 & 8.2 & 8.3 & 8.2 & 8.1 & 8.2 \\
\hline 21 & 8.2 & 8.2 & 8.2 & -- & -- & -- & 8.2 & 8.2 & 8.2 & 8.3 & 8.2 & 8.2 \\
\hline 22 & 8.2 & 8.1 & 8.2 & --- & - - - & --- & 8.3 & 8.2 & 8.2 & 8.2 & 8.0 & 8.1 \\
\hline 23 & 8.2 & 8.1 & 8.1 & -- & -- & --- & 8.2 & 8.0 & 8.2 & 8.2 & 7.9 & 8.1 \\
\hline 24 & 8.2 & 8.1 & 8.1 & -- & -- & --- & 8.3 & 8.1 & 8.2 & 8.2 & 8.0 & 8.2 \\
\hline 25 & 8.1 & 8.1 & 8.1 & -- & -- & -- & 8.3 & 8.1 & 8.2 & 8.3 & 8.0 & 8.1 \\
\hline 26 & 8.2 & 8.0 & 8.1 & -- & -- & -- & 8.3 & 8.1 & 8.2 & 8.3 & 8.2 & 8.3 \\
\hline 27 & 8.1 & 8.1 & 8.1 & -- & -- & --- & 8.3 & 8.1 & 8.2 & 8.3 & 8.2 & 8.2 \\
\hline 28 & 8.2 & 8.0 & 8.1 & -- & -- & --- & 8.3 & 8.2 & 8.3 & 8.2 & 8.2 & 8.2 \\
\hline 29 & 8.3 & 8.2 & 8.2 & - . & - - - & --- & 8.3 & 8.2 & 8.2 & 8.3 & 8.2 & 8.2 \\
\hline 30 & 8.2 & 8.1 & 8.2 & 8.3 & 8.2 & 8.3 & 8.3 & 8.1 & 8.2 & 8.3 & 8.1 & 8.2 \\
\hline 31 & 8.2 & 8.2 & 8.2 & --- & --- & --- & 8.3 & 8.1 & 8.2 & 8.3 & 8.2 & 8.3 \\
\hline MONTH & --- & -. & --- & -- &.- & --- & 8.3 & 8.0 & 8.2 & 8.3 & 7.9 & 8.2 \\
\hline
\end{tabular}

\begin{tabular}{|c|c|c|c|}
\hline \multirow[t]{2}{*}{ DAY } & MAX & MIN & MEAN \\
\hline & \multicolumn{3}{|c|}{ FEBRUARY 2000} \\
\hline 1 & 8.3 & 8.2 & 8.2 \\
\hline 2 & 8.3 & 7.8 & 8.2 \\
\hline 3 & 8.3 & 7.9 & 8.1 \\
\hline 4 & 8.3 & 8.0 & 8.2 \\
\hline 5 & 8.3 & 8.2 & 8.3 \\
\hline 6 & 8.3 & 8.1 & 8.2 \\
\hline 7 & 8.3 & 7.9 & 8.2 \\
\hline 8 & 8.2 & 8.0 & 8.1 \\
\hline 9 & 8.2 & 7.8 & 8.1 \\
\hline 10 & 8.2 & 8.1 & 8.2 \\
\hline 11 & 8.2 & 7.9 & 8.1 \\
\hline 12 & 8.2 & 7.8 & 8.0 \\
\hline 13 & 8.2 & 8.0 & 8.1 \\
\hline 14 & 8.2 & 8.0 & 8.1 \\
\hline 15 & 8.2 & 8.1 & 8.1 \\
\hline 16 & 8.2 & 8.1 & 8.2 \\
\hline 17 & 8.2 & 8.1 & 8.2 \\
\hline 18 & 8.2 & 8.1 & 8.1 \\
\hline 19 & 8.2 & 8.1 & 8.2 \\
\hline 20 & 8.2 & 8.2 & 8.2 \\
\hline 21 & 8.2 & 8.0 & 8.1 \\
\hline 22 & 8.2 & 8.0 & 8.1 \\
\hline 23 & 8.2 & 7.9 & 8.1 \\
\hline 24 & 8.1 & 7.9 & 8.1 \\
\hline 25 & 8.1 & 7.9 & 8.1 \\
\hline 26 & 8.2 & 7.9 & 8.1 \\
\hline 27 & 8.2 & 7.9 & 8.1 \\
\hline 28 & 8.2 & 7.9 & 8.1 \\
\hline 29 & 8.1 & 7.4 & 7.8 \\
\hline 30 & -- & --- & -- \\
\hline 31 & -- &.- & $\ldots$ \\
\hline
\end{tabular}


Table 3. Maximum, minimum, and mean specific conductance, $\mathrm{pH}$, water temperature, and dissolved oxygen in Christmas Bay, February 1999-February 2000-Continued

TEMPERATURE, IN DEGREES CELSIUS

\begin{tabular}{|c|c|c|c|c|c|c|c|c|c|c|c|c|}
\hline DAY & MAX & MIN & MEAN & MAX & MIN & MEAN & MAX & MIN & MEAN & MAX & MIN & MEAN \\
\hline & \multicolumn{3}{|c|}{ FEBRUARY 1999} & \multicolumn{3}{|c|}{ MARCH 1999} & \multicolumn{3}{|c|}{ APRIL 1999} & \multicolumn{3}{|c|}{ MAY 1999} \\
\hline 1 & 18.3 & 16.2 & 17.4 & 21.3 & 18.6 & 19.9 & 22.5 & 20.2 & 21.3 & 25.0 & 23.4 & 24.1 \\
\hline 2 & 18.2 & 17.7 & 17.9 & 21.5 & 20.1 & 20.8 & 22.7 & 21.7 & 22.2 & 23.7 & 22.2 & 23.0 \\
\hline 3 & 18.4 & 17.2 & 17.8 & 20.6 & 17.8 & 18.9 & 23.0 & 22.0 & 22.4 & 23.8 & 22.5 & 23.1 \\
\hline 4 & 19.0 & 18.3 & 18.6 & 19.0 & 16.9 & 18.0 & 23.6 & 22.3 & 23.0 & 25.0 & 23.4 & 24.1 \\
\hline 5 & 19.3 & 18.1 & 18.7 & 19.6 & 18.4 & 19.1 & 24.4 & 23.1 & 23.5 & 27.0 & 24.6 & 25.6 \\
\hline 6 & 20.9 & 19.1 & 19.9 & 21.9 & 19.5 & 20.5 & 24.4 & 22.9 & 23.9 & 26.7 & 24.7 & 25.7 \\
\hline 7 & 21.9 & 20.4 & 21.1 & 21.4 & 18.5 & 19.5 & 25.9 & 23.6 & 24.5 & 25.7 & 23.7 & 24.7 \\
\hline 8 & 23.2 & 21.4 & 22.3 & 20.1 & 18.4 & 19.2 & 26.5 & 24.5 & 25.4 & 26.6 & 24.0 & 25.2 \\
\hline 9 & 23.7 & 21.9 & 22.8 & 21.5 & 19.9 & 20.6 & 26.5 & 24.8 & 25.6 & 27.7 & 25.6 & 26.5 \\
\hline 10 & 24.2 & 22.5 & 23.3 & 21.6 & 20.6 & 21.1 & 26.6 & 24.6 & 25.6 & 26.9 & 25.4 & 26.3 \\
\hline 11 & 23.6 & 20.5 & 22.8 & 21.4 & 20.9 & 21.1 & 28.2 & 26.1 & 27.0 & 26.8 & 24.7 & 25.8 \\
\hline 12 & 20.5 & 15.3 & 17.0 & 22.8 & 21.0 & 21.8 & 27.8 & 26.1 & 26.9 & 26.5 & 25.4 & 25.9 \\
\hline 13 & 15.6 & 13.0 & 14.4 & 22.4 & 16.0 & 19.7 & 26.8 & 25.3 & 26.0 & 27.3 & 24.8 & 25.9 \\
\hline 14 & 16.0 & 13.7 & 14.9 & 16.0 & 11.8 & 13.2 & 26.2 & 25.0 & 25.6 & 28.5 & 26.1 & 27.1 \\
\hline 15 & 17.2 & 14.7 & 15.9 & 14.8 & 10.5 & 12.7 & 25.6 & 20.8 & 23.0 & 28.6 & 26.6 & 27.5 \\
\hline 16 & 18.7 & 16.6 & 17.5 & 18.0 & 14.0 & 16.2 & 20.8 & 18.0 & 18.9 & 28.8 & 26.7 & 27.6 \\
\hline 17 & 19.0 & 17.9 & 18.5 & 19.8 & 17.5 & 18.6 & 19.4 & 16.3 & 17.7 & 28.8 & 27.0 & 27.8 \\
\hline 18 & 19.7 & 17.8 & 18.7 & 21.0 & 19.3 & 20.1 & 20.7 & 17.5 & 19.0 & 28.1 & 26.0 & 26.7 \\
\hline 19 & 19.0 & 17.4 & 18.3 & 21.1 & 20.2 & 20.7 & 22.5 & 18.9 & 20.6 & 27.9 & 23.5 & 26.7 \\
\hline 20 & 18.6 & 17.5 & 18.0 & 20.8 & 19.2 & 20.2 & 24.0 & 20.7 & 22.3 & 28.7 & 26.4 & 27.5 \\
\hline 21 & 18.0 & 15.8 & 16.7 & 20.1 & 17.8 & 19.0 & 24.0 & 21.2 & 22.7 & 29.0 & 26.6 & 27.8 \\
\hline 22 & 15.8 & 14.3 & 15.0 & 20.9 & 18.4 & 19.6 & 25.6 & 22.8 & 23.9 & 29.6 & 26.8 & 28.1 \\
\hline 23 & 16.7 & 14.8 & 15.7 & 21.3 & 19.5 & 20.4 & 27.0 & 24.5 & 25.6 & 29.4 & 27.0 & 28.2 \\
\hline 24 & 17.9 & 15.6 & 16.8 & 23.4 & 20.6 & 21.9 & 26.7 & 25.5 & 26.1 & 29.5 & 27.4 & 28.3 \\
\hline 25 & 19.3 & 17.3 & 18.3 & 23.4 & 22.0 & 22.7 & 26.8 & 25.4 & 26.0 & 29.7 & 27.5 & 28.5 \\
\hline 26 & 20.8 & 19.0 & 19.9 & 22.0 & 20.1 & 21.0 & 26.3 & 24.8 & 25.7 & 29.7 & 27.1 & 28.3 \\
\hline 27 & 21.9 & 20.2 & 21.0 & 20.1 & 18.9 & 19.5 & 26.6 & 24.3 & 25.2 & 29.9 & 27.6 & 28.7 \\
\hline 28 & 21.2 & 19.3 & 20.3 & 19.6 & 18.8 & 19.2 & 28.5 & 25.5 & 26.7 & 29.0 & 27.0 & 27.8 \\
\hline 29 & - - &.- &.- & 19.6 & 19.1 & 19.4 & 28.7 & 26.4 & 27.4 & 27.4 & 26.0 & 26.7 \\
\hline 30 & -- & -- & -- & 19.1 & 18.8 & 18.9 & 27.5 & 25.0 & 26.0 & 27.8 & 25.6 & 26.5 \\
\hline 31 & --- & --- & --- & 20.9 & 18.7 & 19.6 & --- & --- & --- & 29.3 & 26.7 & 27.8 \\
\hline MONTH & 24.2 & 13.0 & 18.6 & 23.4 & 10.5 & 19.5 & 28.7 & 16.3 & 24.0 & 29.9 & 22.2 & 26.6 \\
\hline \multirow[t]{2}{*}{ DAY } & MAX & MIN & MEAN & MAX & MIN & MEAN & MAX & MIN & MEAN & MAX & MIN & MEAN \\
\hline & \multicolumn{3}{|c|}{ JUNE 1999} & \multicolumn{3}{|c|}{ JULY 1999} & \multicolumn{3}{|c|}{ AUGUST 1999} & \multicolumn{3}{|c|}{ SEPTEMBER 1999} \\
\hline 1 & 30.0 & 27.8 & 28.8 & 30.0 & 27.6 & 28.8 & 31.9 & 29.2 & 30.5 & 30.6 & 29.0 & 29.7 \\
\hline 2 & 29.6 & 27.9 & 28.6 & 30.1 & 28.0 & 29.1 & 32.2 & 29.8 & 31.0 & 30.7 & 28.8 & 29.6 \\
\hline 3 & 29.6 & 28.0 & 28.8 & 30.5 & 28.3 & 29.3 & 32.6 & 30.3 & 31.4 & 29.8 & 28.6 & 29.2 \\
\hline 4 & 30.6 & 28.3 & 29.3 & 30.6 & 28.5 & 29.4 & 32.3 & 30.1 & 31.2 & 30.4 & 28.0 & 29.0 \\
\hline 5 & 30.4 & 28.2 & 29.3 & 30.4 & 28.9 & 29.6 & 32.4 & 30.2 & 31.3 & 31.1 & 28.9 & 29.9 \\
\hline 6 & 30.6 & 28.4 & 29.3 & 30.4 & 28.9 & 29.4 & 33.0 & 30.5 & 31.6 & 31.6 & 29.5 & 30.4 \\
\hline 7 & 30.7 & 28.8 & 29.6 & 30.4 & 28.3 & 29.2 & 33.0 & 30.8 & 31.8 & 31.9 & 29.7 & 30.7 \\
\hline 8 & 29.9 & 29.0 & 29.4 & 31.3 & 28.9 & 30.0 & 31.7 & 30.3 & 30.9 & 31.7 & 29.6 & 30.6 \\
\hline 9 & 29.5 & 28.1 & 28.8 & 30.4 & 28.0 & 29.0 & 31.5 & 29.5 & 30.4 & 31.6 & 29.7 & 30.6 \\
\hline 10 & 30.4 & 27.9 & 28.9 & 29.6 & 27.2 & 28.3 & 31.1 & 28.9 & 30.0 & 31.0 & 29.4 & 30.2 \\
\hline 11 & 30.0 & 28.4 & 29.2 & 30.3 & 28.4 & 29.1 & 31.5 & 28.9 & 30.2 & 30.9 & 29.0 & 29.9 \\
\hline 12 & 29.5 & 28.4 & 29.0 & 31.3 & 28.9 & 30.0 & 31.5 & 29.2 & 30.4 & 30.6 & 28.6 & 29.5 \\
\hline 13 & 29.2 & 27.9 & 28.5 & 30.2 & 28.8 & 29.5 & 31.3 & 29.1 & 30.2 & 30.0 & 28.5 & 29.3 \\
\hline 14 & 29.5 & 27.7 & 28.6 & 30.2 & 27.8 & 29.0 & 31.8 & 28.8 & 30.3 & 29.7 & 28.0 & 28.8 \\
\hline 15 & 28.8 & 27.8 & 28.3 & 30.6 & 28.0 & 29.2 & 31.3 & 29.8 & 30.5 & 28.9 & 27.4 & 28.2 \\
\hline 16 & 29.1 & 27.5 & 28.2 & 31.1 & 28.8 & 29.9 & 31.2 & 29.1 & 30.1 & 28.4 & 26.8 & 27.6 \\
\hline 17 & 30.0 & 25.9 & 28.9 & 31.1 & 29.0 & 30.0 & 32.1 & 29.7 & 30.8 & 28.2 & 26.5 & 27.3 \\
\hline 18 & 29.3 & 27.6 & 28.5 & 31.3 & 29.3 & 30.2 & 32.6 & 30.0 & 31.2 & 28.0 & 26.2 & 27.0 \\
\hline 19 & 29.5 & 27.5 & 28.5 & 31.3 & 29.3 & 30.3 & 32.5 & 30.2 & 31.2 & 27.8 & 25.8 & 26.7 \\
\hline 20 & 29.3 & 27.7 & 28.5 & 30.5 & 29.3 & 29.8 & 32.0 & 29.9 & 30.9 & 27.9 & 25.8 & 26.8 \\
\hline 21 & 29.0 & 27.9 & 28.5 & 29.3 & 27.6 & 28.1 & 31.5 & 30.0 & 30.7 & 27.5 & 26.1 & 26.9 \\
\hline 22 & 29.5 & 27.8 & 28.7 & 30.2 & 27.2 & 28.5 & 30.6 & 29.1 & 29.7 & 26.1 & 24.3 & 24.9 \\
\hline 23 & 29.5 & 27.8 & 28.7 & 31.5 & 28.6 & 29.8 & 29.8 & 28.4 & 29.0 & 24.6 & 23.1 & 23.9 \\
\hline 24 & -- & -- & -- & 30.4 & 29.3 & 29.8 & 30.8 & 28.7 & 29.6 & 25.5 & 23.3 & 24.2 \\
\hline 25 & --- & --- & --- & 31.1 & 28.9 & 29.7 & 31.7 & 29.4 & 30.3 & 25.4 & 24.0 & 24.7 \\
\hline 26 & 29.6 & 27.5 & 28.5 & 31.5 & 28.8 & 30.1 & 32.1 & 29.8 & 30.8 & 26.9 & 24.8 & 25.7 \\
\hline 27 & 30.5 & 28.1 & 29.2 & 32.0 & 29.2 & 30.5 & 32.2 & 29.7 & 30.9 &.- & - . - & - . \\
\hline 28 & 30.5 & 28.4 & 29.4 & 31.9 & 29.6 & 30.6 & 32.0 & 30.2 & 31.1 & --- & -- & -- \\
\hline 29 & 30.4 & 28.3 & 29.3 & 32.1 & 29.6 & 30.9 & 31.8 & 30.0 & 30.8 & --- & - - - &.- \\
\hline 30 & 29.8 & 27.8 & 28.8 & 31.8 & 29.4 & 30.6 & 31.7 & 29.5 & 30.6 &.-- & -- & -- \\
\hline 31 & --- & --- & --- & 31.5 & 28.9 & 30.2 & 31.0 & 29.9 & 30.4 & --- & -- & -.- \\
\hline MONTH & -- & --- & --- & 32.1 & 27.2 & 29.6 & 33.0 & 28.4 & 30.6 & --- & -. & - \\
\hline
\end{tabular}


Table 3. Maximum, minimum, and mean specific conductance, $\mathrm{pH}$, water temperature, and dissolved oxygen in Christmas Bay, February 1999-February 2000-Continued

TEMPERATURE， IN DEGREES CELSIUS

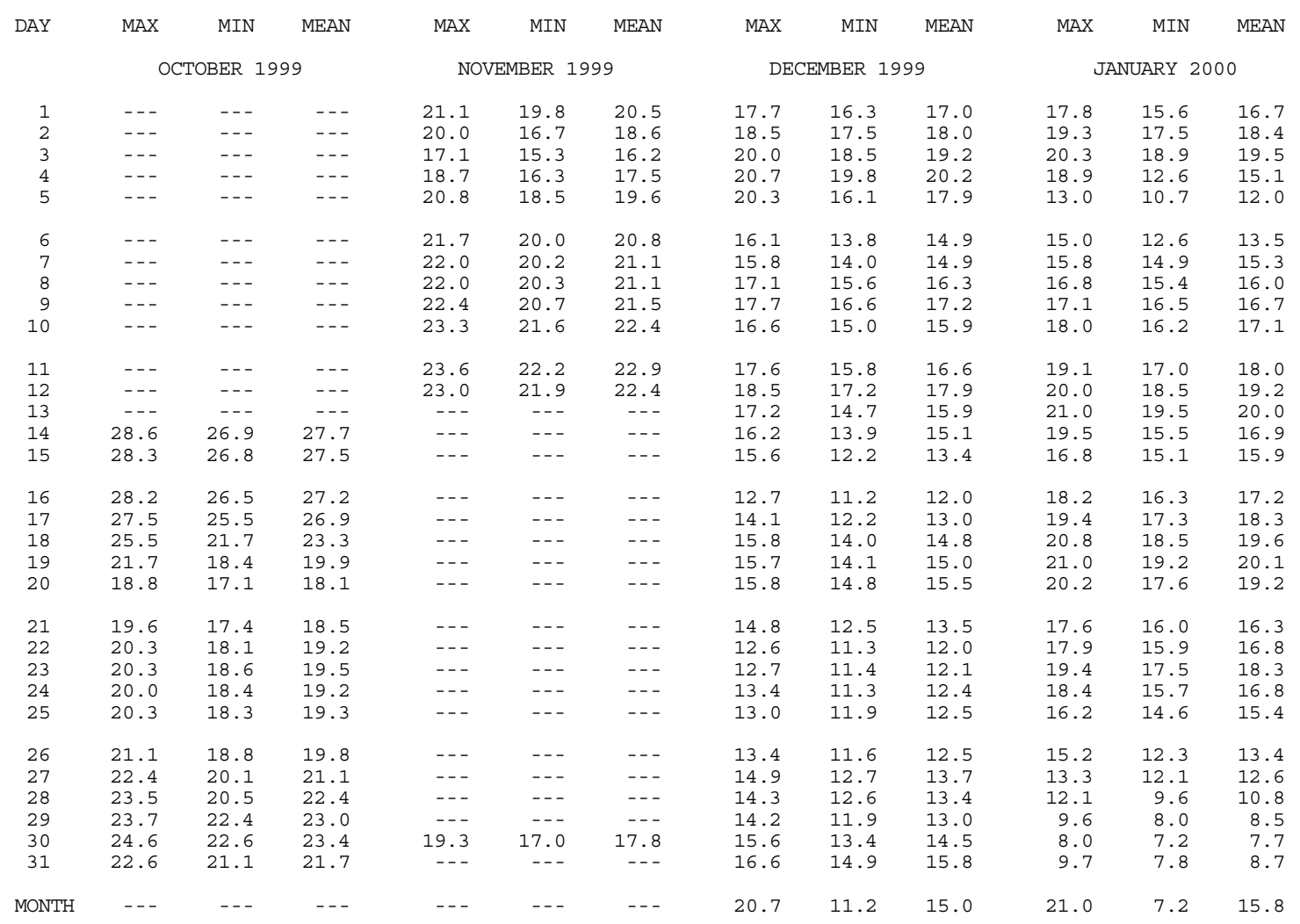

DAY MAX MIN MEAN

FEBRUARY 2000

$\begin{array}{rrrr}1 & 10.8 & 9.6 & 10.2 \\ 2 & 10.9 & 10.2 & 10.6 \\ 3 & 12.9 & 10.3 & 11.5 \\ 4 & 14.3 & 12.1 & 13.0 \\ 5 & 13.4 & 11.4 & 12.4 \\ & & & \\ 6 & 13.4 & 11.7 & 12.6 \\ 7 & 15.4 & 13.3 & 14.3 \\ 8 & 16.9 & 14.6 & 15.7 \\ 9 & 18.1 & 15.7 & 16.8 \\ 10 & 19.2 & 16.7 & 17.9 \\ & & & \\ 11 & 20.3 & 18.1 & 19.2 \\ 12 & 21.1 & 19.6 & 20.4 \\ 13 & 20.9 & 19.9 & 20.4 \\ 14 & 21.5 & 19.9 & 20.6 \\ 15 & 22.0 & 20.2 & 21.1 \\ 16 & 21.5 & 21.0 & 21.3 \\ 17 & 22.7 & 20.8 & 21.6 \\ 18 & 23.2 & 21.3 & 22.2 \\ 19 & 22.7 & 19.2 & 20.6 \\ 20 & 19.2 & 17.6 & 18.2 \\ & & & \\ 21 & 19.3 & 17.1 & 18.2 \\ 22 & 20.3 & 18.3 & 19.2 \\ 23 & 21.1 & 19.2 & 20.1 \\ 24 & 22.1 & 20.0 & 21.0 \\ 25 & 21.9 & 20.8 & 21.4 \\ 26 & 21.4 & 19.9 & 20.9 \\ 27 & 19.9 & 17.9 & 19.0 \\ 28 & 20.2 & 18.0 & 19.2 \\ 29 & 22.8 & 19.1 & 20.0 \\ 30 & --- & --- & --- \\ 31 & --- & --- & --- \\ & & & \\ \text { MONTH } & 23.2 & 9.6 & 17.9\end{array}$


Table 3. Maximum, minimum, and mean specific conductance, $\mathrm{pH}$, water temperature, and dissolved oxygen in Christmas Bay, February 1999-February 2000-Continued DISSOLVED OXYGEN, IN MILLIGRAMS PER LITER

\begin{tabular}{|c|c|c|c|c|c|c|c|c|c|c|c|c|}
\hline DAY & MAX & MIN & MEAN & MAX & MIN & MEAN & MAX & MIN & MEAN & MAX & MIN & MEAN \\
\hline & \multicolumn{3}{|c|}{ FEBRUARY 1999} & \multicolumn{3}{|c|}{ MARCH 1999} & \multicolumn{3}{|c|}{ APRIL 1999} & \multicolumn{3}{|c|}{ MAY 1999} \\
\hline 1 & 8.4 & 6.8 & 7.6 & 7.4 & 5.0 & 6.3 & 6.5 & 5.3 & 6.0 & 6.2 & 5.2 & 5.9 \\
\hline 2 & 8.4 & 6.9 & 7.7 & 7.2 & 5.2 & 6.0 & 6.6 & 5.1 & 5.9 & 6.4 & 5.8 & 6.0 \\
\hline 3 & 8.4 & 6.8 & 7.7 & 7.3 & 5.8 & 6.5 & 6.5 & 4.6 & 5.8 & 6.1 & 5.1 & 5.8 \\
\hline 4 & 8.6 & 6.3 & 7.6 & 7.6 & 6.3 & 7.0 & 6.3 & 4.2 & 5.6 & 7.8 & 4.9 & 6.2 \\
\hline 5 & 8.1 & 7.2 & 7.7 & 7.4 & 5.4 & 6.1 & 6.2 & 4.0 & 4.8 & 7.8 & 2.7 & 5.3 \\
\hline 6 & 7.6 & 5.9 & 6.9 & --- & --- & --- & 6.3 & 3.5 & 5.3 & 7.3 & 2.2 & 5.3 \\
\hline 7 & 7.8 & 5.8 & 6.9 & -- & -- & --- & 5.6 & 4.2 & 4.9 & 7.6 & 4.3 & 6.1 \\
\hline 8 & 7.4 & 6.2 & 6.8 & -- & --- & --- & 5.7 & 4.4 & 5.0 & 7.0 & 5.3 & 6.4 \\
\hline 9 & 7.6 & 5.9 & 6.7 & --- & --- & --- & 5.8 & 3.6 & 4.9 & 7.9 & 3.9 & 6.1 \\
\hline 10 & 7.6 & 6.3 & 6.9 & --- & --- & --- & 5.5 & 2.0 & 4.2 & 6.7 & 3.5 & 5.9 \\
\hline 11 & 8.7 & 6.1 & 7.2 & --- & --- & --- & 5.0 & 1.6 & 3.7 & 7.8 & 4.8 & 6.3 \\
\hline 12 & 9.7 & 7.2 & 8.9 & 6.5 & 4.3 & 5.2 & 5.8 & 3.6 & 4.9 & 8.0 & 3.0 & 6.0 \\
\hline 13 & 10.6 & 7.3 & 9.4 & 7.4 & 3.2 & 6.3 & 5.8 & 4.2 & 5.1 & -- & -- & --- \\
\hline 14 & 11.1 & 8.8 & 10.3 & 9.1 & 6.6 & 8.0 & 6.3 & 4.9 & 5.7 & -- & -- & -- \\
\hline 15 & 10.7 & 8.4 & 10.0 & 8.3 & 5.1 & 6.7 & 6.8 & 3.4 & 6.0 & --- & --- & -- \\
\hline 16 & 9.4 & 7.5 & 8.8 & 7.2 & 3.5 & 6.0 & 6.9 & 3.3 & 6.1 & --- & --- & -- \\
\hline 17 & 9.5 & 7.4 & 8.5 & -- & --- & --- & 7.7 & 3.4 & 5.8 & -- & -- & --- \\
\hline 18 & 9.6 & 7.2 & 8.4 & --- & --- & --- & 7.6 & 4.8 & 6.5 &.-- &.-- & -- \\
\hline 19 & 9.5 & 8.0 & 9.0 & -- & --- & --- & 7.5 & 5.9 & 6.5 & -- & -- & --- \\
\hline 20 & 10.1 & 8.0 & 9.2 & -- & --- & --- & 6.3 & 5.7 & 6.0 & 5.6 & 4.3 & 5.1 \\
\hline 21 & 10.3 & 8.8 & 9.7 & -- & -- & --- & 8.6 & 5.5 & 6.2 & 5.6 & 4.4 & 5.2 \\
\hline 22 & 10.8 & 10.0 & 10.4 & --- & --- & --- & 6.3 & 4.5 & 5.5 & 5.3 & 4.3 & 4.8 \\
\hline 23 & 10.4 & 8.0 & 9.3 & -- & -- & --- & 6.6 & 2.7 & 4.8 & 5.6 & 3.8 & 4.6 \\
\hline 24 & 10.2 & 8.6 & 9.4 & 6.3 & 5.2 & 5.8 & 5.9 & 2.6 & 4.9 & 5.4 & 2.3 & 3.7 \\
\hline 25 & 8.9 & 6.3 & 7.6 & 6.6 & 5.1 & 5.8 & 5.6 & 1.1 & 3.9 & 5.5 & 2.2 & 4.4 \\
\hline 26 & 7.1 & 5.7 & 6.5 & 6.6 & 6.0 & 6.4 & 6.1 & 1.3 & 4.0 & 5.4 & 2.1 & 4.2 \\
\hline 27 & 7.6 & 5.2 & 6.5 & 6.9 & 6.1 & 6.6 & 4.9 & 1.2 & 2.6 & 4.7 & 2.0 & 3.8 \\
\hline 28 & 7.4 & 5.7 & 6.5 & 7.0 & 4.8 & 6.0 & 5.4 & 1.9 & 3.5 & 5.2 & 2.0 & 3.5 \\
\hline 29 & --- & -- & --- & 7.0 & 3.9 & 5.8 & 5.9 & 2.4 & 4.3 & 5.4 & 2.6 & 3.9 \\
\hline 30 & -- & --- & -- & 6.6 & 5.2 & 5.8 & 6.0 & 3.0 & 5.6 & 5.5 & 3.3 & 4.3 \\
\hline 31 & --- & --- & --- & 6.8 & 5.0 & 5.8 & --- & --- & --- & 4.4 & 2.0 & 3.6 \\
\hline MONTH & 11.1 & 5.2 & 8.1 & --- & --- & --- & 8.6 & 1.1 & 5.1 & --- & --- & --- \\
\hline \multirow[t]{2}{*}{ DAY } & MAX & MIN & MEAN & MAX & MIN & MEAN & MAX & MIN & MEAN & MAX & MIN & MEAN \\
\hline & \multicolumn{3}{|c|}{ JUNE 1999} & \multicolumn{3}{|c|}{ JULY 1999} & \multicolumn{3}{|c|}{ AUGUST 1999} & \multicolumn{3}{|c|}{ SEPTEMBER 1999} \\
\hline 1 & 4.4 & 3.0 & 3.8 & 4.6 & 2.9 & 4.0 & 4.0 & 1.6 & 2.9 & 5.9 & 2.4 & 4.2 \\
\hline 2 & 4.2 & 2.4 & 3.3 & 4.3 & 2.4 & 3.5 & 3.4 & .4 & 1.9 & 6.1 & 3.1 & 5.1 \\
\hline 3 & 4.6 & 1.9 & 3.3 & 4.1 & .4 & 2.9 & 3.9 & .3 & 2.0 & 5.7 & 2.7 & 4.4 \\
\hline 4 & 4.6 & 3.0 & 4.0 & 3.9 & .3 & 2.2 & 3.4 & .3 & 2.1 & 6.4 & 4.0 & 5.3 \\
\hline 5 & 4.7 & 3.0 & 4.1 & 2.5 & .2 & 1.0 & 3.3 & 1.1 & 2.2 & 6.3 & 3.7 & 4.8 \\
\hline 6 & 4.5 & 2.2 & 3.4 & 3.3 & .2 & 1.7 & 2.7 & .3 & 1.3 & 5.3 & 2.5 & 4.0 \\
\hline 7 & 4.1 & .7 & 2.7 & 3.0 & .8 & 2.1 & 3.4 & .8 & 2.0 & 5.7 & 2.8 & 4.5 \\
\hline 8 & 4.3 & 2.1 & 3.2 & 3.6 & 1.5 & 2.8 & 3.6 & 1.1 & 2.4 & 6.0 & 2.7 & 4.6 \\
\hline 9 & 4.3 & 1.9 & 3.5 & 3.7 & 2.4 & 3.2 & 4.7 & 2.8 & 3.6 & 5.8 & 2.7 & 4.3 \\
\hline 10 & 4.4 & 2.8 & 3.8 & 4.6 & 1.5 & 2.9 & 4.9 & 2.9 & 3.8 & 5.1 & 3.4 & 4.6 \\
\hline 11 & 4.1 & 2.7 & 3.6 & 3.5 & .4 & 2.2 & 4.8 & 2.7 & 3.7 & 5.8 & 3.6 & 4.9 \\
\hline 12 & 4.0 & 2.2 & 3.3 & 4.0 & .2 & 2.4 & 4.4 & 2.7 & 3.6 & 5.8 & 4.5 & 5.2 \\
\hline 13 & 4.3 & .9 & 2.8 & 4.8 & 2.0 & 2.9 & 4.5 & 2.4 & 3.7 & 6.2 & 3.4 & 5.2 \\
\hline 14 & 4.7 & .9 & 2.5 & 4.1 & 1.4 & 3.2 & 4.4 & .5 & 2.6 & 6.1 & 4.6 & 5.5 \\
\hline 15 & 4.7 & 1.1 & 2.7 & 4.3 & 2.0 & 3.4 & 4.1 & .4 & 2.7 & 6.1 & 4.2 & 5.3 \\
\hline 16 & 3.9 & 1.5 & 2.8 & 4.7 & 1.1 & 3.0 & 3.8 & 1.1 & 2.3 & 6.3 & 3.1 & 5.5 \\
\hline 17 & 7.1 & 2.1 & 4.2 & 4.5 & .3 & 2.3 & 2.9 & .3 & 1.5 & 6.1 & 3.1 & 5.0 \\
\hline 18 & 5.3 & 4.5 & 4.8 & 4.1 & 1.9 & 2.9 & 3.6 & .4 & 1.9 & 6.1 & 2.1 & 5.0 \\
\hline 19 & 5.0 & 3.9 & 4.4 & 5.3 & 2.2 & 3.5 & 3.1 & .3 & 1.8 & 6.0 & 3.0 & 4.6 \\
\hline 20 & 4.8 & 3.5 & 4.1 & 5.7 & 1.2 & 3.2 & 3.5 & .4 & 2.2 & 6.1 & 3.5 & 4.8 \\
\hline 21 & 4.5 & 1.8 & 3.4 & 5.3 & 2.7 & 3.6 & 3.7 & .3 & 1.8 & 6.7 & 4.5 & 5.8 \\
\hline 22 & 4.7 & 1.7 & 3.5 & 4.2 & 1.4 & 2.2 & 3.6 & 2.0 & 3.1 & 6.6 & 5.5 & 6.2 \\
\hline 23 & 5.0 & 3.6 & 4.2 & 3.5 & .9 & 2.3 & 4.1 & 1.5 & 3.2 & 6.3 & 4.9 & 5.7 \\
\hline 24 & -- & -- & -- & 3.5 & 1.7 & 2.6 & 6.6 & 1.5 & 4.2 & 6.3 & 3.8 & 5.4 \\
\hline 25 & --- & --- & --- & 3.9 & 1.5 & 2.5 & 6.4 & 3.5 & 5.1 & 6.5 & 4.6 & 5.7 \\
\hline 26 & 5.1 & 3.5 & 4.5 & 4.0 & .3 & 2.7 & 6.4 & 1.7 & 4.5 & 6.1 & 3.8 & 5.3 \\
\hline 27 & 4.8 & 4.1 & 4.4 & 4.2 & 1.0 & 2.7 & 6.2 & 3.2 & 4.6 & -- & -- & --- \\
\hline 28 & 4.7 & 3.7 & 4.2 & 4.6 & 1.3 & 3.1 & 5.0 & 2.2 & 3.9 & -- & -- & -- \\
\hline 29 & 4.3 & 2.6 & 3.5 & 4.2 & 1.9 & 3.2 & 5.8 & 1.6 & 3.2 & --- & -- & --- \\
\hline 30 & 4.8 & 2.7 & 4.1 & 4.3 & 2.6 & 3.6 & 5.5 & 1.3 & 3.5 & -- & -- & --- \\
\hline 31 & --- & --- & --- & 4.7 & 2.7 & 3.8 & 5.6 & 2.4 & 4.1 & --- & --- & --- \\
\hline MONTH & --- & --- & --- & 5.7 & .2 & 2.8 & 6.6 & .3 & 2.9 & -- & -- & --- \\
\hline
\end{tabular}


Table 3. Maximum, minimum, and mean specific conductance, $\mathrm{pH}$, water temperature, and dissolved oxygen in Christmas Bay, February 1999-February 2000-Continued

DISSOLVED OXYGEN, IN MILLIGRAMS PER LITER

\begin{tabular}{|c|c|c|c|c|c|c|c|c|c|c|c|c|}
\hline \multirow[t]{2}{*}{ DAY } & MAX & MIN & MEAN & MAX & MIN & MEAN & MAX & MIN & MEAN & MAX & MIN & MEAN \\
\hline & \multicolumn{3}{|c|}{ OCTOBER 1999} & \multicolumn{3}{|c|}{ NOVEMBER 1999} & \multicolumn{3}{|c|}{ DECEMBER 1999} & \multicolumn{3}{|c|}{ JANUARY 2000} \\
\hline 1 & -- & -- & --- & 6.6 & 4.4 & 5.7 & 9.3 & 8.4 & 8.8 & 9.7 & 8.0 & 9.0 \\
\hline 2 & -- & -- & --- & 6.8 & 5.2 & 6.2 & 8.9 & 8.4 & 8.6 & 9.1 & 7.9 & 8.7 \\
\hline 3 & -- & -- & --- & 7.2 & 6.1 & 6.7 & 8.5 & 8.0 & 8.2 & 8.7 & 6.9 & 7.9 \\
\hline 4 & -- & -- & --- & 7.0 & 6.6 & 6.8 & 8.2 & 6.6 & 7.6 & 10.3 & 7.5 & 9.5 \\
\hline 5 & --- & --- & --- & 6.7 & 6.0 & 6.3 & 8.7 & 6.7 & 8.1 & 11.2 & 9.9 & 10.7 \\
\hline 6 & --- & --- & --- & 6.5 & 5.1 & 6.0 & 9.7 & 7.6 & 8.9 & 10.9 & 9.6 & 10.4 \\
\hline 7 & -- & -- & --- & 6.3 & 4.6 & 5.5 & 10.3 & 6.7 & 9.0 & 10.4 & 9.1 & 9.7 \\
\hline 8 & --- & -- & --- & 6.2 & 4.4 & 5.5 & 10.0 & 7.4 & 8.9 & 9.9 & 6.2 & 8.4 \\
\hline 9 & --- & --- & --- & 6.5 & 4.7 & 5.7 & 9.0 & 7.8 & 8.3 & 8.8 & 5.0 & 7.3 \\
\hline 10 & --- & --- & --- & 6.2 & 4.3 & 5.5 & 9.8 & 7.5 & 8.8 & 9.5 & 4.9 & 8.2 \\
\hline 11 & -- & -- & --- & 6.2 & 5.1 & 5.6 & 9.5 & 8.1 & 8.8 & 9.1 & 5.1 & 7.6 \\
\hline 12 & -- & -- & --- & 6.0 & 4.9 & 5.5 & 9.2 & 7.7 & 8.4 & 8.4 & 6.9 & 7.8 \\
\hline 13 & --- & -- & --- & -- & --- & --- & 9.7 & 8.2 & 8.8 & 8.8 & 4.8 & 6.9 \\
\hline 14 & 6.2 & 4.8 & 5.5 & -- &.-- & --- & 9.8 & 8.3 & 9.0 & 9.7 & 8.5 & 9.3 \\
\hline 15 & 6.1 & 4.7 & 5.5 & --- & --- & --- & 10.7 & 9.2 & 10.1 & 9.5 & 7.7 & 8.9 \\
\hline 16 & 6.0 & 5.1 & 5.5 & -- & -- & --- & 11.0 & 9.4 & 10.1 & 9.1 & 6.5 & 8.3 \\
\hline 17 & 5.7 & 4.4 & 5.1 & -- & -- & --- & 11.1 & 9.2 & 10.3 & 9.0 & 6.9 & 8.1 \\
\hline 18 & 5.6 & 5.0 & 5.3 & -- & -- & --- & 10.6 & 7.8 & 9.7 & 9.0 & 6.6 & 7.8 \\
\hline 19 & 6.3 & 5.5 & 6.0 &.- & -- & -- & 10.2 & 8.8 & 9.7 & 8.6 & 7.3 & 7.9 \\
\hline 20 & 6.6 & 5.0 & 5.9 & --- & --- & --- & 10.1 & 9.4 & 9.7 & 8.6 & 6.1 & 8.1 \\
\hline 21 & 6.8 & 4.9 & 5.7 & -- & -- & -- & 10.6 & 9.0 & 10.0 & 9.3 & 7.8 & 8.8 \\
\hline 22 & 6.6 & 4.6 & 5.6 & --- & --- & --- & 11.6 & 10.4 & 11.2 & 9.0 & 5.3 & 7.7 \\
\hline 23 & 5.9 & 4.3 & 5.1 & -- & -- & --- & 11.8 & 8.2 & 11.0 & 9.2 & 4.5 & 7.4 \\
\hline 24 & 5.6 & 4.1 & 5.1 & -- & -- & -- & 11.9 & 8.4 & 10.8 & 9.1 & 6.2 & 8.0 \\
\hline 25 & 5.1 & 4.0 & 4.7 & --- & --- & --- & 12.2 & 9.8 & 11.3 & 9.5 & 5.8 & 7.8 \\
\hline 26 & 4.9 & 3.5 & 4.3 &.-- & -.- & --- & 11.5 & 9.3 & 10.6 & 10.7 & 8.3 & 10.1 \\
\hline 27 & 4.8 & 3.4 & 4.2 & -- & --- & --- & 11.9 & 8.9 & 10.3 & 10.9 & 9.9 & 10.6 \\
\hline 28 & 8.0 & 3.7 & 6.0 &.- & -- & -- & 11.2 & 9.9 & 10.6 & 11.9 & 10.6 & 11.2 \\
\hline 29 & 6.9 & 4.9 & 6.2 & -- & -- & -- & 11.1 & 9.6 & 10.4 & 13.2 & 11.7 & 12.7 \\
\hline 30 & 6.9 & 5.4 & 6.1 & 9.2 & 8.6 & 8.9 & 10.4 & 8.4 & 9.6 & 13.9 & 11.8 & 13.4 \\
\hline 31 & 6.7 & 4.8 & 5.5 & --- & --- & --- & 10.3 & 8.4 & 9.4 & 13.7 & 12.3 & 13.3 \\
\hline MONTH & -- & -- & --- & -- & -- & --- & 12.2 & 6.6 & 9.5 & 13.9 & 4.5 & 9.1 \\
\hline
\end{tabular}

\begin{tabular}{|c|c|c|c|}
\hline \multirow[t]{2}{*}{ DAY } & MAX & MIN & MEAN \\
\hline & \multicolumn{3}{|c|}{ FEBRUARY 2000} \\
\hline 1 & 12.6 & 11.9 & 12.3 \\
\hline 2 & 12.2 & 5.6 & 10.9 \\
\hline 3 & 11.7 & 4.5 & 8.6 \\
\hline 4 & 11.6 & 5.9 & 10.0 \\
\hline 5 & 11.5 & 10.1 & 10.9 \\
\hline 6 & 11.1 & 9.0 & 10.4 \\
\hline 7 & 10.2 & 5.9 & 9.1 \\
\hline 8 & 9.8 & 5.1 & 8.3 \\
\hline 9 & 9.4 & 4.3 & 7.4 \\
\hline 10 & 9.1 & 7.3 & 8.2 \\
\hline 11 & 8.2 & 4.4 & 6.8 \\
\hline 12 & 8.1 & 3.8 & 6.5 \\
\hline 13 & 8.1 & 5.4 & 6.8 \\
\hline 14 & 8.0 & 3.6 & 6.0 \\
\hline 15 & 7.7 & 5.0 & 6.7 \\
\hline 16 & 7.9 & 4.8 & 6.7 \\
\hline 17 & 8.0 & 5.8 & 7.3 \\
\hline 18 & 7.8 & 4.3 & 6.6 \\
\hline 19 & 8.5 & 4.3 & 7.9 \\
\hline 20 & 9.2 & 8.2 & 8.7 \\
\hline 21 & 8.8 & 6.1 & 7.9 \\
\hline 22 & 8.7 & 6.0 & 7.9 \\
\hline 23 & 8.3 & 4.1 & 6.7 \\
\hline 24 & 7.7 & 2.3 & 6.3 \\
\hline 25 & 7.7 & 4.0 & 6.5 \\
\hline 26 & 7.6 & 2.2 & 5.6 \\
\hline 27 & 8.3 & 3.4 & 6.4 \\
\hline 28 & 8.4 & 2.2 & 6.7 \\
\hline 29 & 10.2 & 5.1 & 7.0 \\
\hline 30 & --- & --- & --- \\
\hline 31 & -- & -- & -- \\
\hline MONTH & 12.6 & 2.2 & 7.8 \\
\hline
\end{tabular}


Table 4. Monthly and periodic water-quality data in Christmas, Bastrop, and Drum Bays, February 1999-January 2000

[MG/L, milligrams per liter; US/CM, microsiemens per centimeter at 25 degrees Celsius; DEG C, degrees Celsius; <, less than;

V, contamination; E, estimated; COLS./100 ML, colonies per 100 milliliters; UG/L, micrograms per liter; >, greater than]

CHRISTMAS BAY

\begin{tabular}{|c|c|c|c|c|c|c|c|c|c|c|c|c|c|}
\hline DATE & TIME & $\begin{array}{c}\text { OXYGEN, } \\
\text { DIS- } \\
\text { SOLVED } \\
\text { (MG/L) }\end{array}$ & $\begin{array}{c}\text { PH } \\
\text { WATER } \\
\text { WHOLE } \\
\text { FIELD } \\
\text { (STAND- } \\
\text { ARD } \\
\text { UNITS) }\end{array}$ & $\begin{array}{l}\text { SPE- } \\
\text { CIFIC } \\
\text { CON- } \\
\text { DUCT- } \\
\text { ANCE } \\
\text { (US/CM) }\end{array}$ & $\begin{array}{c}\text { TEMPER- } \\
\text { ATURE } \\
\text { WATER } \\
\text { (DEG C) }\end{array}$ & $\begin{array}{l}\text { CALCIUM } \\
\text { DIS- } \\
\text { SOLVED } \\
\text { (MG/L } \\
\text { AS CA) }\end{array}$ & $\begin{array}{l}\text { MAGNE- } \\
\text { SIUM, } \\
\text { DIS- } \\
\text { SOLVED } \\
\text { (MG/L } \\
\text { AS MG) }\end{array}$ & $\begin{array}{l}\text { POTAS- } \\
\text { SIUM, } \\
\text { DIS- } \\
\text { SOLVED } \\
\text { (MG/L } \\
\text { AS K) }\end{array}$ & $\begin{array}{l}\text { SODIUM, } \\
\text { DIS- } \\
\text { SOLVED } \\
\text { (MG/L } \\
\text { AS NA) }\end{array}$ & $\begin{array}{c}\text { ALKA- } \\
\text { LINITY } \\
\text { WAT DIS } \\
\text { TOT IT } \\
\text { FIELD } \\
\text { (MG/L AS } \\
\text { CACO3) }\end{array}$ & $\begin{array}{c}\text { BICAR- } \\
\text { BONATE } \\
\text { WATER } \\
\text { DIS IT } \\
\text { FIELD } \\
\text { (MG/L AS } \\
\text { HCO3) }\end{array}$ & $\begin{array}{l}\text { CHLO- } \\
\text { RIDE, } \\
\text { DIS- } \\
\text { SOLVED } \\
\text { (MG/L } \\
\text { AS CL) }\end{array}$ & $\begin{array}{l}\text { FLUO- } \\
\text { RIDE, } \\
\text { DIS- } \\
\text { SOLVEI } \\
\text { (MG/L } \\
\text { AS F) }\end{array}$ \\
\hline \multicolumn{14}{|l|}{ FEB 1999} \\
\hline $25 \ldots$ & 0951 & 7.0 & 8.0 & 43100 & 17.6 & 373 & 1180 & 306 & 9550 & -- & -- & 15700 & .6 \\
\hline \multicolumn{14}{|l|}{ MAR } \\
\hline $17 \ldots$ & 0845 & 7.1 & 7.6 & 47100 & 17.6 & 399 & 1250 & 334 & 10300 & 102 & 120 & 17100 & .6 \\
\hline \multicolumn{14}{|l|}{ APR } \\
\hline $21 \ldots$ & 1012 & 6.4 & 8.1 & 42000 & 21.7 & 872 & 1140 & 304 & 9450 & 112 & 139 & 14700 & 1.1 \\
\hline \multicolumn{14}{|l|}{ MAY } \\
\hline $19 \ldots$ & 1110 & 6.0 & 8.3 & 42800 & 26.0 & 357 & 1100 & 278 & 9260 & 104 & 127 & 14800 & .6 \\
\hline \multicolumn{14}{|l|}{ JUN } \\
\hline $17 \ldots$ & 1030 & 4.5 & 7.9 & 46800 & 28.1 & 382 & 1220 & 374 & 9640 & 122 & 140 & 17200 & .9 \\
\hline \multicolumn{14}{|l|}{ JUL } \\
\hline $16 \ldots$ & 0934 & 4.2 & 8.5 & 44000 & 28.8 & 382 & 1180 & 315 & 9320 & 116 & 142 & 15600 & .6 \\
\hline \multicolumn{14}{|l|}{ AUG } \\
\hline $24 \ldots$ & 1202 & 4.7 & 8.1 & 57800 & 29.8 & 405 & 1290 & 438 & 10600 & 132 & 161 & 20500 & .7 \\
\hline \multicolumn{14}{|l|}{ SEP } \\
\hline $27 \ldots$ & 0954 & 4.4 & 8.1 & 56400 & 26.0 & 423 & 1380 & 434 & 11000 & 150 & 183 & 20400 & .6 \\
\hline \multicolumn{14}{|l|}{ OCT } \\
\hline $28 \ldots$ & 0954 & 5.8 & 8.2 & 50400 & 21.6 & 367 & 1140 & 339 & 9160 & 98 & 128 & 17500 & .6 \\
\hline \multicolumn{14}{|l|}{ NOV } \\
\hline $17 \ldots$ & 0928 & 6.5 & 8.1 & 51900 & 20.0 & 381 & 1180 & 378 & 9600 & 108 & 138 & 18100 & .7 \\
\hline \multicolumn{14}{|l|}{$\mathrm{DEC}$} \\
\hline $\begin{array}{c}16 \ldots \\
\text { JAN } 2000\end{array}$ & 0923 & 7.0 & 7.9 & 50700 & 11.3 & 371 & 1150 & 406 & 9630 & 101 & 123 & 17400 & .5 \\
\hline $21 \ldots$ & 0918 & 7.7 & 7.7 & 52700 & 15.8 & 368 & 1150 & 358 & 9520 & 120 & 147 & 18100 & .6 \\
\hline
\end{tabular}

\begin{tabular}{|c|c|c|c|c|c|c|c|c|c|c|c|c|c|}
\hline DATE & $\begin{array}{l}\text { SILICA, } \\
\text { DIS- } \\
\text { SOLVED } \\
\text { (MG/L } \\
\text { AS } \\
\text { SIO2) }\end{array}$ & $\begin{array}{l}\text { SULFATE } \\
\text { DIS- } \\
\text { SOLVED } \\
\text { (MG/L } \\
\text { AS SO4) }\end{array}$ & $\begin{array}{c}\text { NITRO- } \\
\text { GEN, } \\
\text { AMMONIA } \\
\text { DIS- } \\
\text { SOLVED } \\
\text { (MG/L } \\
\text { AS N) }\end{array}$ & $\begin{array}{l}\text { NITRO- } \\
\text { GEN, AM- } \\
\text { MONIA + } \\
\text { ORGANIC } \\
\text { DIS. } \\
\text { (MG/L } \\
\text { AS N) }\end{array}$ & $\begin{array}{c}\text { NITRO- } \\
\text { GEN, AM- } \\
\text { MONIA + } \\
\text { ORGANIC } \\
\text { TOTAL } \\
\text { (MG/L } \\
\text { AS N) }\end{array}$ & $\begin{array}{c}\text { NITRO- } \\
\text { GEN, } \\
\text { NO2+NO3 } \\
\text { DIS- } \\
\text { SOLVED } \\
\text { (MG/L } \\
\text { AS N) }\end{array}$ & $\begin{array}{c}\text { NITRO- } \\
\text { GEN, } \\
\text { NITRITE } \\
\text { DIS- } \\
\text { SOLVED } \\
\text { (MG/L } \\
\text { AS N) }\end{array}$ & $\begin{array}{c}\text { PHOS- } \\
\text { PHORUS } \\
\text { DIS- } \\
\text { SOLVED } \\
\text { (MG/L } \\
\text { AS P) }\end{array}$ & $\begin{array}{c}\text { PHOS- } \\
\text { PHORUS } \\
\text { ORTHO, } \\
\text { DIS- } \\
\text { SOLVED } \\
\text { (MG/L } \\
\text { AS P) }\end{array}$ & $\begin{array}{c}\text { PHOS- } \\
\text { PHORUS } \\
\text { TOTAL } \\
\text { (MG/L } \\
\text { AS P) }\end{array}$ & $\begin{array}{c}\text { CARBON, } \\
\text { ORGANIC } \\
\text { TOTAL } \\
(\text { MG/L } \\
\text { AS C) }\end{array}$ & $\begin{array}{c}\text { PLANK- } \\
\text { TON } \\
\text { BIOMASS } \\
\text { ASH WT } \\
(\text { MG/L) }\end{array}$ & $\begin{array}{c}\text { PLANK- } \\
\text { TON } \\
\text { BIOMASS } \\
\text { DRY WT } \\
\text { (MG/L) }\end{array}$ \\
\hline \multicolumn{14}{|l|}{ FEB 1999} \\
\hline $25 \ldots$ & $<5.0$ & 2050 & $<.020$ & .22 & .26 & $<.050$ & .019 & .021 & .015 & .024 & 2.8 & 123 & 126 \\
\hline \multicolumn{14}{|l|}{ MAR } \\
\hline $17 \ldots$ & $<5.0$ & 2250 & .021 & .16 & .36 & $<.050$ & $<.010$ & .017 & .016 & .038 & V2. 8 & 283 & 291 \\
\hline \multicolumn{14}{|l|}{ APR } \\
\hline $21 \ldots$ & 5.4 & 1940 & .024 & .23 & -- & .074 & $<.010$ & .017 & .023 & .173 & 5.6 & 444 & 466 \\
\hline \multicolumn{14}{|l|}{ MAY } \\
\hline $19 \ldots$ & 3.0 & 2180 & .026 & .26 & .37 & $<.050$ & $<.010$ & .018 & .019 & .039 & 4.2 & 594 & 612 \\
\hline \multicolumn{14}{|l|}{ JUN } \\
\hline $17 \ldots$ & 3.2 & 2250 & .071 & E. 07 & .41 & $<.050$ & $<.010$ & .021 & .025 & .056 & 4.3 & 229 & 238 \\
\hline \multicolumn{14}{|l|}{ JUL } \\
\hline $16 \ldots$ & E3.8 & 2120 & $<.020$ & .13 & .13 & $<.050$ & $<.010$ & .022 & .022 & .041 & 3.0 & 231 & 237 \\
\hline \multicolumn{14}{|l|}{ AUG } \\
\hline $24 \ldots$ & E3. 8 & 2780 & .053 & .21 & .26 & $<.050$ & $<.010$ & .043 & .034 & .056 & 4.2 & 242 & 247 \\
\hline \multicolumn{14}{|l|}{ SEP } \\
\hline $27 \ldots$ & 3.0 & 2890 & $<.020$ & .45 & .24 & $<.050$ & $<.010$ & .030 & .022 & .055 & 3.2 & 165 & 171 \\
\hline \multicolumn{14}{|l|}{ OCT } \\
\hline $28 \ldots$ & 1.6 & 2270 & $<.020$ & .27 & .43 & $<.050$ & $<.010$ & .023 & .014 & .040 & -- & 251 & 259 \\
\hline \multicolumn{14}{|l|}{ NOV } \\
\hline $17 \ldots$ & $<1.0$ & 2580 & $<.020$ & .20 & .32 & $<.050$ & $<.010$ & .020 & .016 & .042 & 2.2 & 280 & 290 \\
\hline \multicolumn{14}{|l|}{$\mathrm{DEC}$} \\
\hline $\begin{array}{c}16 \ldots \\
\text { JAN } 2000\end{array}$ & .6 & 2390 & .033 & .17 & .18 & $<.050$ & $<.010$ & .016 & .019 & .030 & 1.6 & -- & -- \\
\hline $21 \ldots$ & 1.1 & 2240 & .029 & .24 & .49 & $<.050$ & $<.010$ & .015 & .013 & .079 & 3.1 & -- & -- \\
\hline
\end{tabular}


Table 4. Monthly and periodic water-quality data in Christmas, Bastrop, and Drum Bays, February 1999-January 2000Continued

CHRISTMAS BAY-CONTINUED

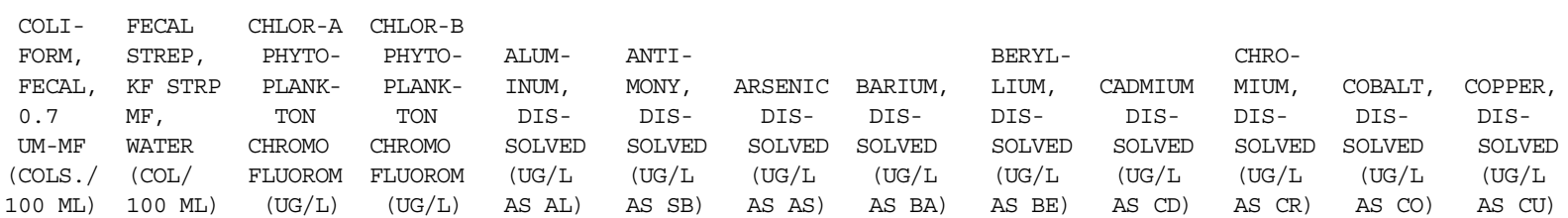

FEB 1999

$25 \ldots$

MAR

$17 \ldots$

APR

$21 \ldots$

MAY

$19 .$.

JUN

JU 17.

$16 \ldots$

AUG

$24 \ldots$

SEP

$27 \ldots$

OCT

$28 \ldots$

NOV

$17 \ldots$

DEC

$16 \ldots$

JAN 2000

$21 \ldots$

1
2
1
1
1
1
2
1
1
1
6
2

6

E.

$2 \quad 3.3<.1$

$66 \quad 1.2<.1$

$54 \quad 1.6<.1$

$30 \quad 4.1<.1$

$1 \quad 1.0<.1$

$\begin{array}{lll}4 & 4.8 & .4\end{array}$

$1 \quad 2.5<.1$

$4 \quad 1.6<.1$

$42<.1$

$6 \quad 2.0<.1$

$<2$

$<20.0<4$

$2.0<.1$

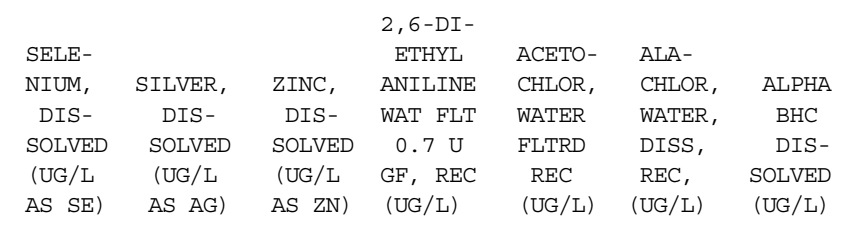

FEB 1999

\begin{tabular}{|c|c|c|c|c|c|c|c|c|c|c|c|c|c|}
\hline $25 \ldots$ & $<500$ & -- & $<200$ & -- & -- & -- & -- & -- & -- & -- & -- & -- & -- \\
\hline \multicolumn{14}{|l|}{ MAR } \\
\hline $17 \ldots$ & $<500$ & -- & $<200$ & $<.10$ & -- & -- & -- & -- & -- & -- & -- & -- & -- \\
\hline \multicolumn{14}{|l|}{ APR } \\
\hline $21 \ldots$ & $<500$ & -- & $<150$ & $<.10$ & -- & -- & -- & -- & -- & $<.003$ & $<.002$ & $<.002$ & $<.002$ \\
\hline \multicolumn{14}{|l|}{ MAY } \\
\hline $19 \ldots$ & $<500$ & $<20.0$ & $<20.0$ & $<.10$ & $<20.0$ & $<20.0$ & 4.7 & $<20.0$ & $<20$ & -- & -- & -- & -- \\
\hline \multicolumn{14}{|l|}{ JUN } \\
\hline $17 \ldots$ & $<250$ & -- & $<75.0$ & $<.10$ & -- & -- & -- & -- & -- & -- & -- & -- & -- \\
\hline \multicolumn{14}{|l|}{ JUL } \\
\hline $16 \ldots$ & $<500$ & -- & $<200$ & $<.10$ & -- & -- & -- & -- & -- & -- & -- & -- & -- \\
\hline \multicolumn{14}{|l|}{ AUG } \\
\hline $24 \ldots$ & $<500$ & -- & $<200$ & $<.10$ & -- & -- & -- & -- & -- & -- & -- & -- & -- \\
\hline \multicolumn{14}{|l|}{ SEP } \\
\hline $27 \ldots$ & $<10$ & -- & 42.3 & $<.10$ & -- & -- & -- & -- & -- & -- & -- & -- & -- \\
\hline \multicolumn{14}{|l|}{ OCT } \\
\hline $28 \ldots$ & $<300$ & -- & $<90.0$ & $<.23$ & -- & -- & -- & -- & -- & -- & -- & -- & -- \\
\hline \multicolumn{14}{|l|}{ NOV } \\
\hline $17 \ldots$ & $<200$ & -- & $<60.0$ & $<.23$ & -- & -- & -- & -- & -- & -- & -- & -- & -- \\
\hline \multicolumn{14}{|l|}{$\mathrm{DEC}$} \\
\hline $16 \ldots$ & $<10$ & $<20.0$ & $<20.0$ & $<.23$ & $<20.0$ & 35.6 & $<4.0$ & $<20.0$ & 33 & -- & -- & -- & -- \\
\hline JAN 2000 & & & & & & & & & & & & & \\
\hline $21 \ldots$ & 20 & -- & $<2.2$ & $<.23$ & -- & -- & -- & -- & -- & -- & -- & -- & -- \\
\hline
\end{tabular}


Table 4. Monthly and periodic water-quality data in Christmas, Bastrop, and Drum Bays, February 1999-January 2000Continued

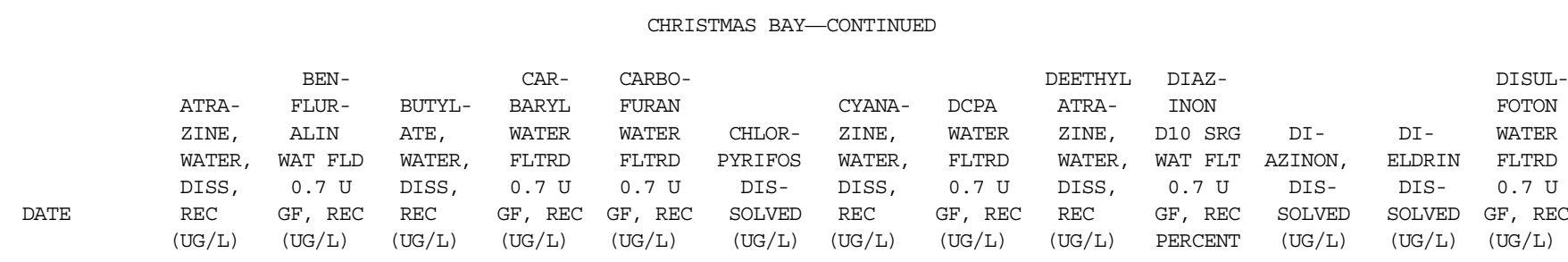

FEB 1999
$25 \ldots$
MAR
$17 \ldots$
APR
$21 \ldots$
MAY
$19 \ldots$
JUN
$17 \ldots$
JUL
$16 \ldots$
AUG
$24 \ldots$
SEP
$27 \ldots$
OCT
$28 \ldots$
NOV
$17 \ldots$
DEC
$16 \ldots$
JAN 2000
$21 \ldots$

\begin{tabular}{|c|c|c|c|c|c|c|c|c|c|c|c|c|c|}
\hline & $\begin{array}{l}\text { EPTC } \\
\text { WATER }\end{array}$ & $\begin{array}{l}\text { ETHAL- } \\
\text { FLUR- } \\
\text { ALIN }\end{array}$ & $\begin{array}{l}\text { ETHO- } \\
\text { PROP } \\
\text { WATER }\end{array}$ & FONOFOS & $\begin{array}{c}\text { HCH } \\
\text { ALPHA } \\
\text { D6 SRG }\end{array}$ & & $\begin{array}{l}\text { LIN- } \\
\text { URON } \\
\text { WATER }\end{array}$ & MALA- & $\begin{array}{c}\text { METHYL } \\
\text { AZIN- } \\
\text { PHOS }\end{array}$ & $\begin{array}{c}\text { METHYL } \\
\text { PARA- } \\
\text { THION }\end{array}$ & METO- & $\begin{array}{l}\text { METRI- } \\
\text { BUZIN }\end{array}$ & $\begin{array}{l}\text { MOL- } \\
\text { INATE } \\
\text { WATER }\end{array}$ \\
\hline & FLTRD & WAT FLT & FLTRD & WATER & WAT FLT & LINDANE & FLTRD & THION, & WAT FLT & WAT FLT & LACHLOR & SENCOR & FLTRD \\
\hline & $0.7 \mathrm{U}$ & $0.7 \mathrm{U}$ & $0.7 \mathrm{U}$ & DISS & $0.7 \mathrm{U}$ & DIS- & $0.7 \mathrm{U}$ & DIS- & $0.7 \mathrm{U}$ & $0.7 \mathrm{U}$ & WATER & WATER & $0.7 \mathrm{U}$ \\
\hline DATE & $\begin{array}{l}\text { GF, REC } \\
\text { (UG/L) }\end{array}$ & $\begin{array}{l}\text { GF， REC } \\
\text { (UG/L) }\end{array}$ & $\begin{array}{l}\text { GF， REC } \\
\text { (UG/L) }\end{array}$ & $\begin{array}{c}\mathrm{REC} \\
\text { (UG/L) }\end{array}$ & $\begin{array}{l}\text { GF， REC } \\
\text { PERCENT }\end{array}$ & $\begin{array}{l}\text { SOLVED } \\
\text { (UG/L) }\end{array}$ & $\begin{array}{l}\text { GF， REC } \\
\text { (UG/L) }\end{array}$ & $\begin{array}{l}\text { SOLVED } \\
\text { (UG/L) }\end{array}$ & $\begin{array}{l}\text { GF, REC } \\
\text { (UG/L) }\end{array}$ & $\begin{array}{l}\text { GF， REC } \\
\text { (UG/L) }\end{array}$ & $\begin{array}{l}\text { DISSOLV } \\
\text { (UG/L) }\end{array}$ & $\begin{array}{l}\text { DISSOLV } \\
\text { (UG/L) }\end{array}$ & $\begin{array}{l}\text { GF, REC } \\
\text { (UG/L) }\end{array}$ \\
\hline
\end{tabular}

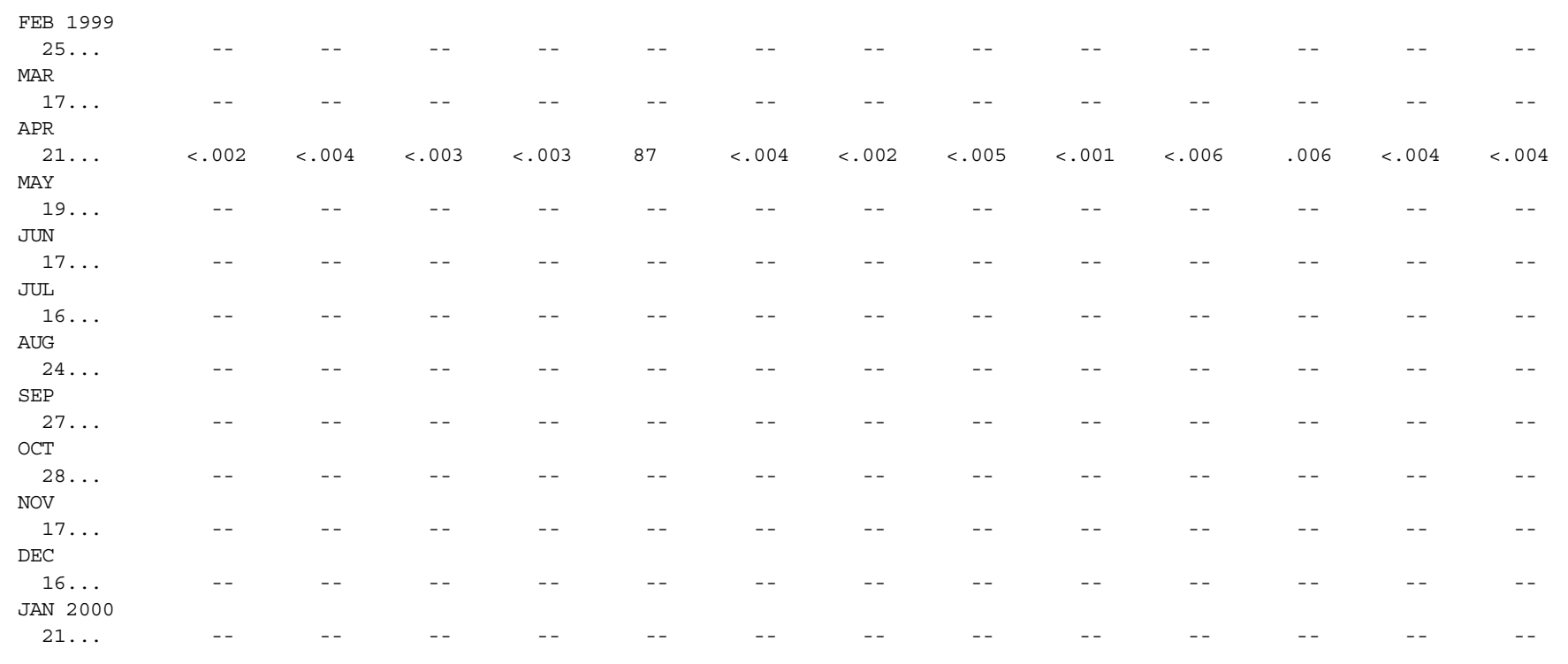


Table 4. Monthly and periodic water-quality data in Christmas, Bastrop, and Drum Bays, February 1999-January 2000Continued

\begin{tabular}{|c|c|c|c|c|c|c|c|c|c|c|c|c|c|}
\hline & $\begin{array}{l}\text { NAPROP- } \\
\text { AMIDE }\end{array}$ & & & $\begin{array}{l}\text { PEB- } \\
\text { ULATE }\end{array}$ & $\begin{array}{l}\text { PENDI- } \\
\text { METH- }\end{array}$ & $\begin{array}{c}\text { PER- } \\
\text { METHRIN }\end{array}$ & & & $\begin{array}{l}\text { PRON- } \\
\text { AMIDE }\end{array}$ & & PRO- & $\begin{array}{c}\text { PRO- } \\
\text { PARGTTF }\end{array}$ & \\
\hline & WATER & & PARA- & WATER & ALIN & $\begin{array}{l}\text { METHRIN } \\
\text { CIS }\end{array}$ & WATER & METON, & $\begin{array}{l}\text { AMIDE } \\
\text { WATER }\end{array}$ & $\begin{array}{l}\text { PROPA- } \\
\text { CHLOR, }\end{array}$ & WATER & $\begin{array}{l}\text { PARGITE } \\
\text { WATER }\end{array}$ & $\begin{array}{l}\text { SI- } \\
\text { MAZINE, }\end{array}$ \\
\hline & $\begin{array}{l}\text { FLTRD } \\
0.7 \mathrm{U}\end{array}$ & $\begin{array}{r}\mathrm{P}^{\prime} \mathrm{P}^{\prime} \\
\mathrm{DDE}\end{array}$ & $\begin{array}{c}\text { THION, } \\
\text { DIS- }\end{array}$ & $\begin{array}{l}\text { FILTRD } \\
0.7 \mathrm{U}\end{array}$ & $\begin{array}{c}\text { WAT FLT } \\
0.7 \mathrm{U}\end{array}$ & $\begin{array}{c}\text { WAT FLT } \\
0.7 \mathrm{U}\end{array}$ & $\begin{array}{l}\text { FLTRD } \\
0.7 \mathrm{U}\end{array}$ & $\begin{array}{l}\text { WATER, } \\
\text { DISS, }\end{array}$ & $\begin{array}{l}\text { FLTRD } \\
0.7 \mathrm{U}\end{array}$ & $\begin{array}{l}\text { WATER, } \\
\text { DISS, }\end{array}$ & $\begin{array}{l}\text { FLTRD } \\
0.7 \mathrm{U}\end{array}$ & $\begin{array}{l}\text { FLTRD } \\
0.7 \mathrm{U}\end{array}$ & $\begin{array}{l}\text { WATER， } \\
\text { DISS, }\end{array}$ \\
\hline $\mathrm{TE}$ & $\begin{array}{l}\text { GF, REC } \\
\text { (UG/L) }\end{array}$ & $\begin{array}{l}\text { DISSOLV } \\
\text { (UG/L) }\end{array}$ & $\begin{array}{l}\text { SOLVED } \\
\text { (UG/L) }\end{array}$ & $\begin{array}{l}\text { GF, REC } \\
\text { (UG/L) }\end{array}$ & $\begin{array}{l}\text { GF, REC } \\
\text { (UG/L) }\end{array}$ & $\begin{array}{l}\text { GF, REC } \\
\text { (UG/L) }\end{array}$ & $\begin{array}{l}\text { GF, REC } \\
\text { (UG/L) }\end{array}$ & $\begin{array}{l}\mathrm{REC} \\
\text { (UG/L) }\end{array}$ & $\begin{array}{l}\text { GF, REC } \\
\text { (UG/L) }\end{array}$ & $\begin{array}{l}\mathrm{REC} \\
(\mathrm{UG} / \mathrm{L})\end{array}$ & $\begin{array}{l}\text { GF, REC } \\
\text { (UG/L) }\end{array}$ & $\begin{array}{l}\text { GF, REC } \\
\text { (UG/L) }\end{array}$ & $\begin{array}{c}\mathrm{REC} \\
\text { (UG/L) }\end{array}$ \\
\hline
\end{tabular}

FEB 1999
$25 \ldots$
MAR
$17 \ldots$
APR
$21 \ldots$
MAY
$19 \ldots$
JUN
$17 \ldots$
JUL
$16 \ldots$
AUG
$24 \ldots$
SEP
$27 \ldots$
OCT
$28 \ldots$
NOV
$17 \ldots$
DEC
$16 \ldots$
JAN 2000

AN 2000

$\begin{array}{cccccclccc} & \text { TEBU- } & \text { TER- } & \text { TER- } & \text { TERBUTH } & \text { THIO- } & \text { TRIAL- } & \text { TRI- } & & \\ & \text { THIURON } & \text { BACIL } & \text { BUFOS } & \text { YLAZINE } & \text { BENCARB } & \text { LATE } & \text { FLUR- } & \text { URANIUM } & \\ & \text { WATER } & \text { WATER } & \text { WATER } & \text { SURROGT } & \text { WATER } & \text { WATER } & \text { ALIN } & \text { NATURAL } & \text { SEDI- } \\ & \text { FLTRD } & \text { FLTRD } & \text { FLTRD } & \text { WAT FLT } & \text { FLTRD } & \text { FLTRD } & \text { WAT FLT } & \text { DIS- } & \text { MENT, } \\ \text { DATE } & 0.7 \mathrm{U} & 0.7 \mathrm{U} & 0.7 \mathrm{U} & 0.7 \mathrm{U} & 0.7 \mathrm{U} & 0.7 \mathrm{U} & 0.7 \mathrm{U} & \text { SOLVED } & \text { SUS- } \\ & \text { GF, REC } & \text { GF, REC } & \text { GF, REC } & \text { GF, REC } & \text { GF, REC } & \text { GF, REC } & \text { GF, REC } & \text { (UG/L } & \text { PENDED } \\ & \text { (UG/L) } & \text { (UG/L) } & \text { (UG/L) } & \text { PERCENT } & \text { (UG/L) } & \text { (UG/L) } & \text { (UG/L) } & \text { AS U) } & \text { (MG/L) }\end{array}$

\begin{tabular}{|c|c|c|c|c|c|c|c|c|}
\hline $\begin{array}{c}\text { FEB } 1999 \\
25 \ldots \\
\text { MAR }\end{array}$ & -- & -- & -- & -- & -- & -- & -- & -- \\
\hline $17 \ldots$ & -- & -- & -- & -- & -- & -- & -- & -- \\
\hline APR & & & & & & & & \\
\hline $21 \ldots$ & $<.010$ & $<.007$ & $<.013$ & 112 & $<.002$ & $<.001$ & $<.002$ & -- \\
\hline MAY & & & & & & & & \\
\hline $19 \ldots$ & -- & -- & -- & -- & -- & -- & -- & $<20.0$ \\
\hline $17 \ldots$ & -- & -- & -- & -- & -- & -- & -- & -- \\
\hline $\begin{array}{l}\text { JUL } \\
\qquad 16 \ldots\end{array}$ & -- & -- & -- & -- & -- & -- & -- & -- \\
\hline AUG & & & & & & & & \\
\hline $\begin{array}{l}24 \ldots \\
\operatorname{SEP}\end{array}$ & -- & -- & -- & -- & -- & -- & -- & -- \\
\hline$\underset{\text { ОСТ }}{27 \ldots}$ & -- & -- & -- & -- & -- & -- & -- & -- \\
\hline$\underset{\text { NOV }}{28 \ldots}$ & -- & -- & -- & -- & -- & -- & -- & -- \\
\hline $\begin{array}{c}17 \ldots \\
\text { DEC }\end{array}$ & -- & -- & -- & -- & -- & -- & -- & -- \\
\hline $\begin{array}{c}16 \ldots \\
\text { JAN } 2000\end{array}$ & -- & -- & -- & -- & -- & -- & -- & $<20.0$ \\
\hline $21 \ldots$ & -- & -- & -- & -- & -- & -- & -- & -- \\
\hline
\end{tabular}


Table 4. Monthly and periodic water-quality data in Christmas, Bastrop, and Drum Bays, February 1999-January 2000Continued

BASTROP BAY

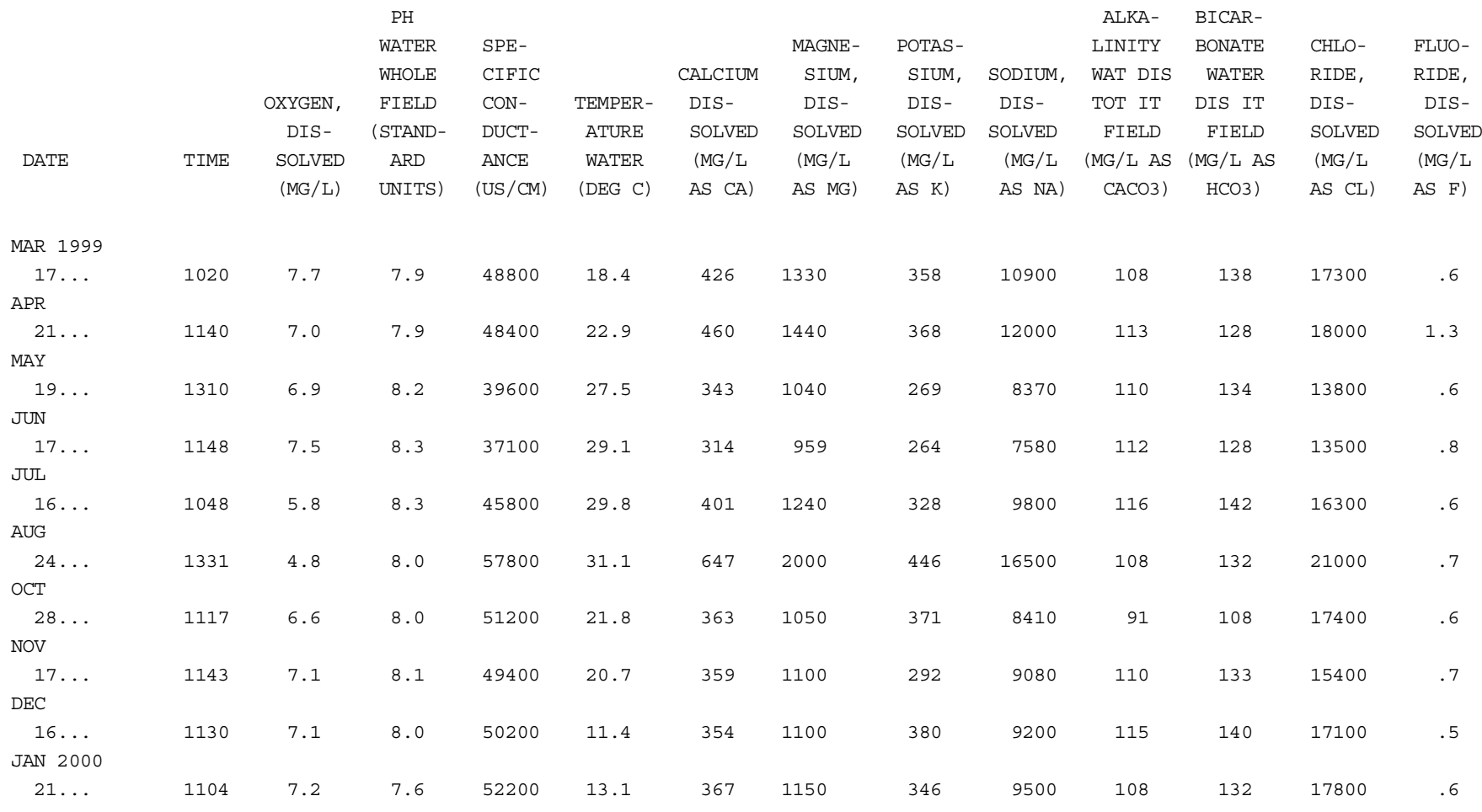

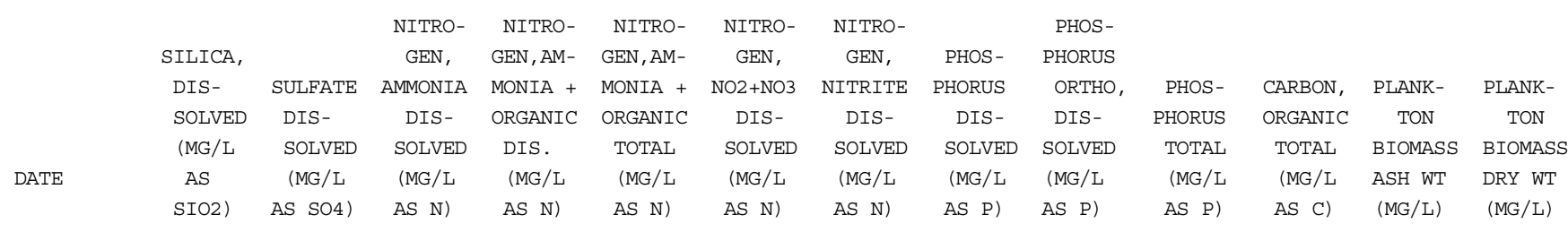

MAR 1999

\begin{tabular}{|c|c|c|c|c|c|c|c|c|c|c|c|c|c|}
\hline $17 \ldots$ & $<5.0$ & 2420 & .042 & .16 & .35 & $<.050$ & $<.010$ & .011 & .012 & .055 & 4.2 & 278 & 287 \\
\hline \multicolumn{14}{|l|}{ APR } \\
\hline $21 \ldots$ & 2.3 & 2380 & .055 & .17 & -- & .097 & $<.010$ & .012 & .022 & .119 & V3. 3 & 610 & 645 \\
\hline \multicolumn{14}{|l|}{ MAY } \\
\hline $19 \ldots$ & $<2.5$ & 2030 & .022 & .28 & .35 & $<.050$ & $<.010$ & .009 & .016 & .029 & 3.0 & 277 & 284 \\
\hline \multicolumn{14}{|l|}{ JUN } \\
\hline $17 \ldots$ & .6 & 1770 & .034 & E. 10 & .47 & $<.050$ & $<.010$ & .011 & .019 & .047 & 5.6 & 233 & 240 \\
\hline \multicolumn{14}{|l|}{ JUL } \\
\hline $16 \ldots$ & $\mathrm{E} 2.7$ & 1890 & $<.020$ & .17 & .15 & $<.050$ & $<.010$ & $<.004$ & .020 & .047 & 3.5 & 256 & 267 \\
\hline \multicolumn{14}{|l|}{ AUG } \\
\hline $24 \ldots$ & E1. 7 & 2850 & .049 & .15 & .22 & $<.050$ & $<.010$ & .016 & .015 & .040 & 3.3 & 76.8 & 78.8 \\
\hline \multicolumn{14}{|l|}{ OCT } \\
\hline $28 \ldots$ & $\mathrm{E} 1.2$ & 2380 & -- & -- & -- & -- & -- & -- & -- & -- & -- & 290 & 304 \\
\hline \multicolumn{14}{|l|}{ NOV } \\
\hline $17 \ldots$ & 1.2 & 2390 & $<.020$ & .16 & .28 & $<.050$ & $<.010$ & .019 & $<.010$ & .036 & 2.1 & 259 & 270 \\
\hline \multicolumn{14}{|l|}{$\mathrm{DEC}$} \\
\hline $16 \ldots$ & $<.1$ & 2390 & .043 & E. 07 & .15 & $<.050$ & $<.010$ & .018 & .013 & .029 & 1.3 & -- & -- \\
\hline \multicolumn{14}{|l|}{ JAN 2000} \\
\hline $21 \ldots$ & 2.5 & 2230 & $<.020$ & .21 & .36 & $<.050$ & $<.010$ & .016 & .018 & .046 & 2.6 & 562 & 578 \\
\hline
\end{tabular}


Table 4. Monthly and periodic water-quality data in Christmas, Bastrop, and Drum Bays, February 1999-January 2000Continued

BASTROP BAY-CONTINUED

\begin{tabular}{|c|c|c|c|c|c|c|c|c|c|c|c|c|}
\hline COLI - & FECAL & CHLOR-A & CHLOR-B & & & & & & & & & \\
\hline FORM， & STREP, & РHYTO- & РHYTO- & ALUM- & ANTI - & & & BERYL- & & CHRO- & & \\
\hline $\begin{array}{l}\text { FECAL， } \\
0.7\end{array}$ & $\begin{array}{l}\mathrm{KF} \text { STRP } \\
\mathrm{MF} \text {, }\end{array}$ & $\begin{array}{c}\text { PLANK- } \\
\text { TON }\end{array}$ & $\begin{array}{c}\text { PLANK- } \\
\text { TON }\end{array}$ & $\begin{array}{r}\text { INUM, } \\
\text { DIS- }\end{array}$ & $\begin{array}{r}\text { MONY， } \\
\text { DIS- }\end{array}$ & $\begin{array}{c}\text { ARSENIC } \\
\text { DIS- }\end{array}$ & $\begin{array}{l}\text { BARIUM, } \\
\text { DIS- }\end{array}$ & $\begin{array}{l}\text { LIUM, } \\
\text { DIS- }\end{array}$ & $\begin{array}{c}\text { CADMIUM } \\
\text { DIS- }\end{array}$ & $\begin{array}{l}\text { MIUM, } \\
\text { DIS- }\end{array}$ & $\begin{array}{l}\text { COBALT, } \\
\text { DIS- }\end{array}$ & $\begin{array}{c}\text { COPPER, } \\
\text { DIS- }\end{array}$ \\
\hline UM-MF & WATER & CHROMO & CHROMO & SOLVED & SOLVED & SOLVED & SOLVED & SOLVED & SOLVED & SOLVED & SOLVED & SOLVED \\
\hline (COLS.) & (COL) & FLUOROM & FLUOROM & (UG/L & (UG/L & (UG/L & (UG/L & (UG/L & (UG/L & (UG/L & (UG/L & (UG/L \\
\hline 100 ML) & 100 ML) & (UG/L) & (UG/L) & AS AL) & AS SB) & AS AS) & AS BA) & AS BE) & AS CD) & AS CR) & $\mathrm{AS} \quad \mathrm{CO})$ & AS CU) \\
\hline
\end{tabular}

MAR 1999

$17 \ldots$

APR

$21 \ldots$

MAY

$19 \ldots$

JUN

$17 \ldots$

JUL

$16 \ldots$

AUG

24 ...

$\mathrm{OCT}$

$28 \ldots$

NOV

$17 \ldots$

DEC

$16 \ldots$

JAN 2000

$21 \ldots$
$1-120$

$4 \quad 64$

4

2

1

18

1

1

64

170

2

110

32

20

2

$1 \quad 150$

$4.0<.1$

$5.0<.1$

30

$2.9<.1$
$1.9<$

4.4

4.8

8.3

$2.6<.1$

$1.8<.1$

$1.4<.1$
$<15<15.0$
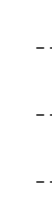$$
-
$$$$
\begin{array}{llllll}
<20.0 & <4.0 \quad 38.0<20.0 & <20.0 & 6.0 & <20.0 & <20.0
\end{array}
$$$$
<20<20.0
$$

\begin{tabular}{|c|c|c|c|c|c|c|c|c|c|c|c|c|}
\hline & & & & & & & & & $2,6-\mathrm{DI}-$ & & & \\
\hline IRON， & LEAD, & $\begin{array}{l}\text { MANGA- } \\
\text { NESE, }\end{array}$ & MERCURY & $\begin{array}{l}\text { MOLYB- } \\
\text { DENUM, }\end{array}$ & NICKEL, & $\begin{array}{l}\text { SELE- } \\
\text { NIUM, }\end{array}$ & SILVER, & ZINC, & $\begin{array}{c}\text { ETHYL } \\
\text { ANILINE }\end{array}$ & $\begin{array}{l}\text { ACETO- } \\
\text { CHLOR, }\end{array}$ & $\begin{array}{l}\text { ALA- } \\
\text { CHLOR, }\end{array}$ & ALPHA \\
\hline DIS- & DIS- & DIS- & DIS- & DIS- & DIS- & DIS- & DIS- & DIS- & WAT FLT & WATER & WATER, & $\mathrm{BHC}$ \\
\hline SOLVED & SOLVED & SOLVED & SOLVED & SOLVED & SOLVED & SOLVED & SOLVED & SOLVED & $0.7 \mathrm{U}$ & FLTRD & DISS, & DIS- \\
\hline (UG/L & (UG/L & (UG/L & (UG/L & (UG/L & (UG/L & (UG/L & (UG/L & (UG/L & GF， REC & $\mathrm{REC}$ & $\mathrm{REC}$, & SOLVED \\
\hline AS FE) & AS PB) & AS $\mathrm{MN}$ ) & AS HG) & AS MO) & AS NI) & AS SE) & AS AG) & AS $\quad \mathrm{ZN})$ & (UG/L) & (UG/L) & (UG/L) & (UG/L) \\
\hline
\end{tabular}

MAR 1999

$17 \ldots$
APR
$21 \ldots$
MAY
$19 \ldots$
JUN
$17 \ldots$
JUL
$16 \ldots$
AUG
$24 \ldots$
OCT
$28 \ldots$
NOV
$17 \ldots$
DEC
$16 \ldots$
JAN 2000

$E 420$
$<500$
$<500$
$<10$
$<500$
$<500$
30
$<200$
$<10$

$--<200$

160 
Table 4. Monthly and periodic water-quality data in Christmas, Bastrop, and Drum Bays, February 1999-January 2000Continued

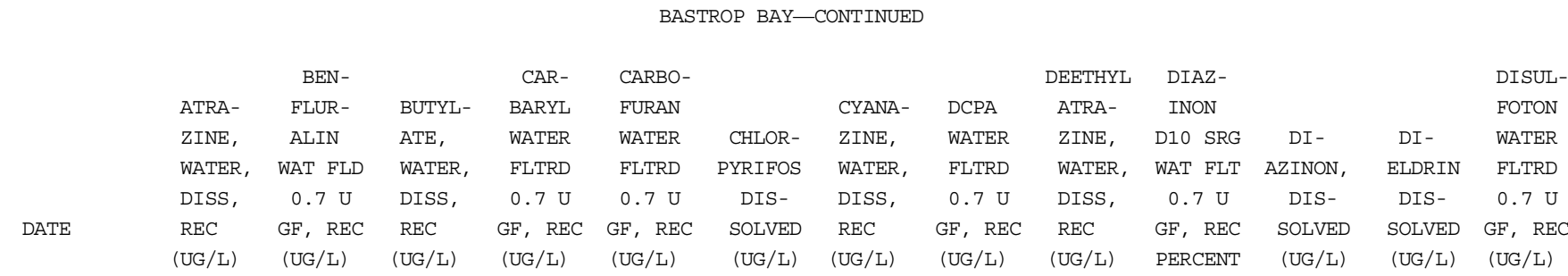

MAR 1999

$17 \ldots$

APR

$21 \ldots$

MAY

$19 \ldots$

JUN

$17 \ldots$

JUL

$16 \ldots$

AUG

$24 \ldots$

OCT

$28 \ldots$

NOV

$17 \ldots$

DEC

$16 \ldots$

JAN 2000

$21 \ldots$

$.029<.002$

$002<.002$

003

003

$<.004$

$<.004$

$<.002$

E. 005

77

$<.002<.001<.017$

\begin{tabular}{|c|c|c|c|c|c|c|c|c|c|c|c|c|c|}
\hline & $\begin{array}{l}\text { EPTC } \\
\text { WATER }\end{array}$ & $\begin{array}{l}\text { ETHAL- } \\
\text { FLUR- } \\
\text { ALIN }\end{array}$ & $\begin{array}{l}\text { ETHO- } \\
\text { PROP } \\
\text { WATER }\end{array}$ & FONOFOS & $\begin{array}{c}\text { HCH } \\
\text { ALPHA } \\
\text { D6 } \quad \text { SRG }\end{array}$ & & $\begin{array}{l}\text { LIN- } \\
\text { URON } \\
\text { WATER }\end{array}$ & MALA- & $\begin{array}{l}\text { METHYL } \\
\text { AZIN- } \\
\text { PHOS }\end{array}$ & $\begin{array}{c}\text { METHYL } \\
\text { PARA- } \\
\text { THION }\end{array}$ & METO- & $\begin{array}{l}\text { METRI- } \\
\text { BUZIN }\end{array}$ & $\begin{array}{l}\text { MOL- } \\
\text { INATE } \\
\text { WATER }\end{array}$ \\
\hline & FLTRD & WAT FLT & FLTRD & WATER & WAT FLT & LINDANE & FLTRD & THION, & WAT FLT & WAT FLT & LACHLOR & SENCOR & FLTRD \\
\hline & $0.7 \mathrm{U}$ & $0.7 \mathrm{U}$ & $0.7 \mathrm{U}$ & DISS & $0.7 \mathrm{U}$ & DIS- & $0.7 \mathrm{U}$ & DIS- & $0.7 \mathrm{U}$ & $0.7 \mathrm{U}$ & WATER & WATER & $0.7 \mathrm{U}$ \\
\hline $\mathrm{TTE}$ & $\begin{array}{l}\text { GF， REC } \\
\text { (UG/L) }\end{array}$ & $\begin{array}{l}\text { GF, REC } \\
\text { (UG/L) }\end{array}$ & $\begin{array}{l}\text { GF， REC } \\
\text { (UG/L) }\end{array}$ & $\begin{array}{c}\mathrm{REC} \\
\text { (UG/L) }\end{array}$ & $\begin{array}{l}\text { GF, REC } \\
\text { PERCENT }\end{array}$ & $\begin{array}{l}\text { SOLVED } \\
\text { (UG/L) }\end{array}$ & $\begin{array}{l}\text { GF, REC } \\
\text { (UG/L) }\end{array}$ & $\begin{array}{l}\text { SOLVED } \\
\text { (UG/L) }\end{array}$ & $\begin{array}{l}\text { GF, REC } \\
\text { (UG/L) }\end{array}$ & $\begin{array}{l}\text { GF, REC } \\
\text { (UG/L) }\end{array}$ & $\begin{array}{l}\text { DISSOLV } \\
\text { (UG/L) }\end{array}$ & $\begin{array}{l}\text { DISSOLV } \\
\text { (UG/L) }\end{array}$ & $\begin{array}{l}\text { GF, REC } \\
(\mathrm{UG} / \mathrm{L})\end{array}$ \\
\hline
\end{tabular}

MAR 1999

$17 \ldots$
APR
$21 \ldots$
MAY
$19 \ldots$
JUN
$17 \ldots$
JUL
$16 \ldots$
AUG
$24 \ldots$
OCT
$28 \ldots$
NOV
$17 \ldots$
DEC
$16 \ldots$
JAN 200
$21 \ldots$

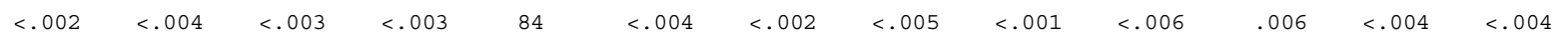

$21 \ldots$ 
Table 4. Monthly and periodic water-quality data in Christmas, Bastrop, and Drum Bays, February 1999-January 2000Continued

\begin{tabular}{|c|c|c|c|c|c|c|c|c|c|c|c|c|c|}
\hline & $\begin{array}{l}\text { NAPROP- } \\
\text { AMIDE }\end{array}$ & & & $\begin{array}{l}\text { PEB- } \\
\text { ULATE }\end{array}$ & $\begin{array}{l}\text { PENDI- } \\
\text { METH- }\end{array}$ & $\begin{array}{c}\text { PER- } \\
\text { METHRIN }\end{array}$ & PHORATE & PRO- & $\begin{array}{l}\text { PRON- } \\
\text { AMIDE }\end{array}$ & PA- & $\begin{array}{l}\text { PRO- } \\
\text { PANIL }\end{array}$ & $\begin{array}{c}\text { PRO- } \\
\text { PARGITE }\end{array}$ & SI- \\
\hline & WATER & & PARA- & WATER & ALIN & CIS & WATER & METON, & WATER & CHLOR, & WATER & WATER & MAZINE, \\
\hline & $\begin{array}{l}\text { FLTRD } \\
0.7 \mathrm{U}\end{array}$ & $\begin{array}{r}\mathrm{P}^{\prime} \mathrm{P}^{\prime} \\
\mathrm{DDE}\end{array}$ & $\begin{array}{c}\text { THION, } \\
\text { DIS- }\end{array}$ & $\begin{array}{l}\text { FILTRD } \\
0.7 \mathrm{U}\end{array}$ & $\begin{array}{c}\text { WAT FLT } \\
0.7 \mathrm{U}\end{array}$ & $\begin{array}{c}\text { WAT FLT } \\
0.7 \mathrm{U}\end{array}$ & $\begin{array}{l}\text { FLTRD } \\
0.7 \mathrm{U}\end{array}$ & $\begin{array}{l}\text { WATER, } \\
\text { DISS, }\end{array}$ & $\begin{array}{l}\text { FLTRD } \\
0.7 \mathrm{U}\end{array}$ & $\begin{array}{l}\text { WATER, } \\
\text { DISS, }\end{array}$ & $\begin{array}{l}\text { FLTRD } \\
0.7 \mathrm{U}\end{array}$ & $\begin{array}{l}\text { FLTRD } \\
0.7 \mathrm{U}\end{array}$ & $\begin{array}{l}\text { WATER, } \\
\text { DISS, }\end{array}$ \\
\hline$E$ & $\begin{array}{l}\text { GF, REC } \\
\text { (UG/L) }\end{array}$ & $\begin{array}{l}\text { DISSOLV } \\
\text { (UG/L) }\end{array}$ & $\begin{array}{l}\text { SOLVED } \\
\text { (UG/L) }\end{array}$ & $\begin{array}{l}\text { GF, REC } \\
\text { (UG/L) }\end{array}$ & $\begin{array}{l}\text { GF, REC } \\
\text { (UG/L) }\end{array}$ & $\begin{array}{l}\text { GF， REC } \\
\text { (UG/L) }\end{array}$ & $\begin{array}{l}\text { GF， REC } \\
\text { (UG/L) }\end{array}$ & $\begin{array}{l}\mathrm{REC} \\
\text { (UG/L) }\end{array}$ & $\begin{array}{l}\text { GF, REC } \\
\text { (UG/L) }\end{array}$ & $\begin{array}{l}\mathrm{REC} \\
\text { (UG/L) }\end{array}$ & $\begin{array}{l}\text { GF, REC } \\
\text { (UG/L) }\end{array}$ & $\begin{array}{l}\mathrm{GF}, \mathrm{REC} \\
\text { (UG/L) }\end{array}$ & $\begin{array}{l}\mathrm{REC} \\
\text { (UG/L) }\end{array}$ \\
\hline
\end{tabular}

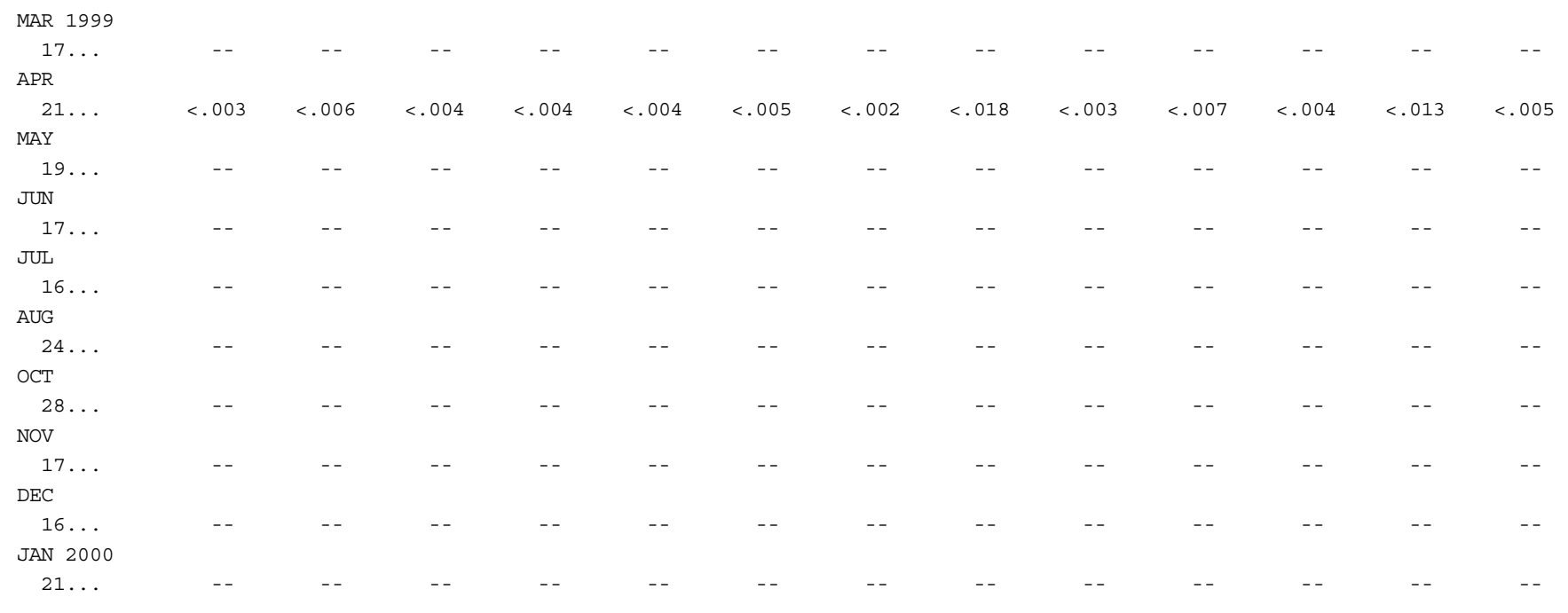

$\begin{array}{cccccccccc} & \text { TEBU- } & \text { TER- } & \text { TER- } & \text { TERBUTH } & \text { THIO- } & \text { TRIAL- } & \text { TRI- } & & \\ \text { THIURON } & \text { BACIL } & \text { BUFOS } & \text { YLAZINE } & \text { BENCARB } & \text { LATE } & \text { FLUR- } & \text { URANIUM } & \\ \text { WATER } & \text { WATER } & \text { WATER } & \text { SURROGT } & \text { WATER } & \text { WATER } & \text { ALIN } & \text { NATURAL } & \text { SEDI- } \\ \text { FLTRD } & \text { FLTRD } & \text { FLTRD } & \text { WAT FLT } & \text { FLTRD } & \text { FLTRD } & \text { WAT FLT } & \text { DIS- } & \text { MENT, } \\ \text { DATE } & 0.7 \mathrm{U} & 0.7 \mathrm{U} & 0.7 \mathrm{U} & 0.7 \mathrm{U} & 0.7 \mathrm{U} & 0.7 \mathrm{U} & 0.7 \mathrm{U} & \text { SOLVED } & \text { SUS- } \\ & \mathrm{GF}, \mathrm{REC} & \mathrm{GF}, \mathrm{REC} & \mathrm{GF}, \mathrm{REC} & \mathrm{GF}, \mathrm{REC} & \mathrm{GF}, \mathrm{REC} & \mathrm{GF}, \mathrm{REC} & \mathrm{GF}, \mathrm{REC} & \text { (UG/L } & \text { PENDED } \\ & \text { (UG/L) } & \text { (UG/L) } & \text { (UG/L) } & \text { PERCENT } & \text { (UG/L) } & \text { (UG/L) } & \text { (UG/L) } & \text { AS U) } & \text { (MG/L) }\end{array}$

\begin{tabular}{|c|c|c|c|c|c|c|c|c|c|}
\hline \multicolumn{10}{|c|}{ MAR 1999} \\
\hline $17 \ldots$ & -- & -- & -- & -- & -- & -- & -- & -- & 41 \\
\hline \multicolumn{10}{|l|}{ APR } \\
\hline $21 \ldots$ & $<.010$ & $<.007$ & $<.013$ & 105 & $<.002$ & $<.001$ & $<.002$ & -- & 128 \\
\hline \multicolumn{10}{|l|}{ MAY } \\
\hline $19 .$. & -- & -- & -- & -- & -- & -- & -- & $<15.0$ & 27 \\
\hline \multicolumn{10}{|l|}{ JUN } \\
\hline $17 \ldots$ & -- & -- & -- & -- & -- & -- & -- & -- & 43 \\
\hline \multicolumn{10}{|l|}{ JUL } \\
\hline $16 \ldots$ & -- & -- & -- & -- & -- & -- & -- & -- & 38 \\
\hline \multicolumn{10}{|l|}{ AUG } \\
\hline $24 \ldots$ & -- & -- & -- & -- & -- & -- & -- & -- & 28 \\
\hline \multicolumn{10}{|l|}{ OCT } \\
\hline $28 \ldots$ & -- & -- & -- & -- & -- & -- & -- & -- & 24 \\
\hline \multicolumn{10}{|l|}{ NOV } \\
\hline $17 \ldots$ & -- & -- & -- & -- & -- & -- & -- & -- & 24 \\
\hline \multicolumn{10}{|l|}{$\mathrm{DEC}$} \\
\hline $16 \ldots$ & -- & -- & -- & -- & -- & -- & -- & $<20.0$ & 21 \\
\hline \multicolumn{10}{|l|}{ JAN 2000} \\
\hline $21 \ldots$ & - - & -- & -- & -- & -- & -- & -- & -- & 62 \\
\hline
\end{tabular}


Table 4. Monthly and periodic water-quality data in Christmas, Bastrop, and Drum Bays, February 1999-January 2000Continued

\begin{tabular}{|c|c|c|c|c|c|c|c|c|c|c|c|c|c|}
\hline & & & & & & DRUM & & & & & & & \\
\hline DATE & TIME & $\begin{array}{c}\text { OXYGEN, } \\
\text { DIS- } \\
\text { SOLVED } \\
\text { (MG/L) }\end{array}$ & $\begin{array}{c}\text { PH } \\
\text { WATER } \\
\text { WHOLE } \\
\text { FIELD } \\
\text { (STAND- } \\
\text { ARD } \\
\text { UNITS) }\end{array}$ & $\begin{array}{l}\text { SPE- } \\
\text { CIFIC } \\
\text { CON- } \\
\text { DUCT- } \\
\text { ANCE } \\
\text { (US/CM) }\end{array}$ & $\begin{array}{c}\text { TEMPER- } \\
\text { ATURE } \\
\text { WATER } \\
\text { (DEG C) }\end{array}$ & $\begin{array}{l}\text { CALCIUM } \\
\text { DIS- } \\
\text { SOLVED } \\
\text { (MG/L } \\
\text { AS CA) }\end{array}$ & $\begin{array}{c}\text { MAGNE- } \\
\text { SIUM, } \\
\text { DIS- } \\
\text { SOLVED } \\
\text { (MG/L } \\
\text { AS MG) }\end{array}$ & $\begin{array}{l}\text { POTAS- } \\
\text { SIUM, } \\
\text { DIS- } \\
\text { SOLVED } \\
\text { (MG/L } \\
\text { AS K) }\end{array}$ & $\begin{array}{l}\text { SODIUM, } \\
\text { DIS- } \\
\text { SOLVED } \\
\text { (MG/L } \\
\text { AS NA) }\end{array}$ & $\begin{array}{c}\text { ALKA- } \\
\text { LINITY } \\
\text { WAT DIS } \\
\text { TOT IT } \\
\text { FIELD } \\
\text { (MG/L AS } \\
\text { CACO3) }\end{array}$ & $\begin{array}{c}\text { BICAR- } \\
\text { BONATE } \\
\text { WATER } \\
\text { DIS IT } \\
\text { FIELD } \\
\text { (MG/L AS } \\
\text { HCO3) }\end{array}$ & $\begin{array}{l}\text { CHLO- } \\
\text { RIDE, } \\
\text { DIS- } \\
\text { SOLVED } \\
\text { (MG/L } \\
\text { AS CL) }\end{array}$ & $\begin{array}{l}\text { FLUO- } \\
\text { RIDE, } \\
\text { DIS- } \\
\text { SOLVED } \\
(\text { MG /L } \\
\text { AS F) }\end{array}$ \\
\hline FEB 1999 & & & & & & & & & & & & & \\
\hline$\underset{\operatorname{MAR}}{25 \ldots}$ & 1121 & 7.8 & 8.0 & 42400 & 19.1 & 359 & 1130 & 298 & 9220 & -- & -- & 15500 & .6 \\
\hline $\begin{array}{c}17 \ldots \\
A P R\end{array}$ & 0926 & 7.0 & 7.7 & 47500 & 18.3 & 414 & 1300 & 174 & 10600 & 111 & 142 & 18200 & .6 \\
\hline$\underset{\operatorname{MAY}}{21 \ldots}$ & 1102 & 6.6 & 7.7 & 44300 & 22.7 & 394 & 1200 & 296 & 10200 & 109 & 133 & 15800 & 1.0 \\
\hline$\underset{\text { JUN }}{19 \ldots}$ & 1205 & 6.4 & 8.1 & 43000 & 26.5 & 377 & 1150 & 256 & 9230 & 113 & 138 & 15000 & .7 \\
\hline $\begin{array}{l}17 \ldots \\
\pi L\end{array}$ & 1108 & 6.2 & 7.9 & 39700 & 28.2 & 330 & 1020 & 281 & 8090 & 93 & 102 & 14500 & .8 \\
\hline $\begin{array}{c}16 \ldots \\
\text { AUG }\end{array}$ & 1008 & 5.0 & 8.2 & 43900 & 29.7 & 375 & 1160 & 317 & 9190 & 106 & 129 & 15300 & .6 \\
\hline$\underset{\text { SEP }}{24 \ldots}$ & 1248 & 4.5 & 7.9 & 57400 & 30.4 & 401 & 1270 & 424 & 10500 & 110 & 134 & 20500 & .7 \\
\hline $\begin{array}{l}27 \ldots \\
\text { OCT }\end{array}$ & 1042 & 5.2 & 8.0 & 60300 & 26.1 & 468 & 1300 & 472 & 12000 & 166 & 203 & 23000 & .6 \\
\hline $\begin{array}{l}28 \ldots \\
\text { NOV }\end{array}$ & 1034 & 6.5 & 8.0 & 52100 & 21.6 & 365 & 1090 & 342 & 8760 & 111 & 142 & 17800 & .6 \\
\hline $\begin{array}{c}17 \ldots \\
\mathrm{DEC}\end{array}$ & 1059 & 5.6 & 8.0 & 51700 & 20.1 & 374 & 1150 & 336 & 9360 & 122 & 141 & 16500 & .7 \\
\hline $\begin{array}{c}16 \ldots \\
\text { JAN } 2000\end{array}$ & 1045 & 8.4 & 7.9 & 50800 & 10.2 & 364 & 1130 & 382 & 9460 & 118 & 144 & 17500 & .5 \\
\hline $21 \ldots$ & 0959 & 6.4 & 7.7 & 52900 & 15.0 & 362 & 1140 & 356 & 9380 & 117 & 143 & 18100 & .6 \\
\hline
\end{tabular}

\begin{tabular}{|c|c|c|c|c|c|c|c|c|c|c|c|c|c|}
\hline DATE & $\begin{array}{l}\text { SILICA, } \\
\text { DIS- } \\
\text { SOLVED } \\
\text { (MG/L } \\
\text { AS } \\
\text { SIO2) }\end{array}$ & $\begin{array}{l}\text { SULFATE } \\
\text { DIS- } \\
\text { SOLVED } \\
\text { (MG/L } \\
\text { AS SO4) }\end{array}$ & $\begin{array}{c}\text { NITRO- } \\
\text { GEN, } \\
\text { AMMONIA } \\
\text { DIS- } \\
\text { SOLVED } \\
\text { (MG/L } \\
\text { AS N) }\end{array}$ & $\begin{array}{l}\text { NITRO- } \\
\text { GEN, AM- } \\
\text { MONIA + } \\
\text { ORGANIC } \\
\text { DIS. } \\
\text { (MG/L } \\
\text { AS N) }\end{array}$ & $\begin{array}{l}\text { NITRO- } \\
\text { GEN, AM- } \\
\text { MONIA + } \\
\text { ORGANIC } \\
\text { TOTAL } \\
\text { (MG/L } \\
\text { AS N) }\end{array}$ & $\begin{array}{c}\text { NITRO- } \\
\text { GEN, } \\
\text { NO2+NO3 } \\
\text { DIS- } \\
\text { SOLVED } \\
\text { (MG/L } \\
\text { AS N) }\end{array}$ & $\begin{array}{c}\text { NITRO- } \\
\text { GEN, } \\
\text { NITRITE } \\
\text { DIS- } \\
\text { SOLVED } \\
\text { (MG/L } \\
\text { AS N) }\end{array}$ & $\begin{array}{c}\text { PHOS- } \\
\text { PHORUS } \\
\text { DIS- } \\
\text { SOLVED } \\
\text { (MG/L } \\
\text { AS P) }\end{array}$ & $\begin{array}{c}\text { PHOS- } \\
\text { PHORUS } \\
\text { ORTHO, } \\
\text { DIS- } \\
\text { SOLVED } \\
\text { (MG/L } \\
\text { AS P) }\end{array}$ & $\begin{array}{c}\text { PHOS- } \\
\text { PHORUS } \\
\text { TOTAL } \\
\text { (MG/L } \\
\text { AS P) }\end{array}$ & $\begin{array}{c}\text { CARBON, } \\
\text { ORGANIC } \\
\text { TOTAL } \\
\text { (MG/L } \\
\text { AS C) }\end{array}$ & $\begin{array}{l}\text { PLANK- } \\
\text { TON } \\
\text { BIOMASS } \\
\text { ASH WT } \\
\text { (MG/L) }\end{array}$ & $\begin{array}{l}\text { PLANK- } \\
\text { TON } \\
\text { BIOMASS } \\
\text { DRY WT } \\
\text { (MG/L) }\end{array}$ \\
\hline FEB 1999 & & & & & & & & & & & & & \\
\hline$\underset{M A R}{25 \ldots}$ & $<5.0$ & 2020 & .037 & .22 & .34 & $<.050$ & .023 & .027 & .016 & .053 & 3.1 & 233 & 239 \\
\hline $\begin{array}{l}17 \ldots \\
A P R\end{array}$ & $<5.0$ & 2340 & .065 & .21 & .42 & .055 & $<.010$ & .030 & .027 & .069 & V2.5 & 670 & 692 \\
\hline$\stackrel{21 \ldots}{\operatorname{MAY}}$ & 3.3 & 2180 & .060 & .26 & .38 & .087 & $<.010$ & .024 & .029 & $<.050$ & 7.4 & 896 & 966 \\
\hline $\operatorname{JUN}^{19} \ldots$ & 3.3 & 2110 & .028 & .29 & .42 & $<.050$ & $<.010$ & .033 & .018 & .055 & 3.9 & 386 & 402 \\
\hline $\begin{array}{l}17 \ldots \\
\text { JUL }\end{array}$ & 3.5 & 1910 & .067 & E. 07 & .43 & .053 & .010 & .013 & .016 & .053 & 4.6 & 221 & 228 \\
\hline $\begin{array}{l}16 \ldots \\
\text { AUG }\end{array}$ & E3. 5 & 1830 & $<.020$ & .20 & .20 & $<.050$ & .015 & .020 & .025 & .057 & 3.1 & 236 & 244 \\
\hline $\begin{array}{l}24 \ldots \\
\operatorname{SEP}\end{array}$ & E2. 8 & 2810 & .034 & .23 & .25 & $<.050$ & $<.010$ & .037 & .033 & .071 & 4.1 & 258 & 264 \\
\hline $\begin{array}{l}27 \ldots \\
\text { ОСТ }\end{array}$ & 5.5 & 3180 & .042 & .35 & .49 & $<.050$ & $<.010$ & .018 & .011 & .070 & 6.1 & 433 & 467 \\
\hline $\begin{array}{l}28 \ldots \\
\text { NOV }\end{array}$ & 1.6 & 2320 & .053 & .30 & .45 & $<.050$ & $<.010$ & .035 & .024 & .054 & 3.8 & 287 & 299 \\
\hline$\underset{\mathrm{DEC}}{17 \ldots}$ & 1.3 & 2560 & -- & -- & -- & -- & -- & -- & -- & -- & -- & 284 & 295 \\
\hline $\begin{array}{c}16 \ldots \\
\text { JAN } 2000\end{array}$ & .4 & 2380 & .048 & .15 & .19 & $<.050$ & $<.010$ & .021 & .019 & .035 & 1.4 & -- & -- \\
\hline $21 \ldots$ & 1.6 & 2260 & $<.020$ & .26 & .45 & $<.050$ & $<.010$ & .012 & .013 & .088 & 3.4 & 1020 & 1050 \\
\hline
\end{tabular}


Table 4. Monthly and periodic water-quality data in Christmas, Bastrop, and Drum Bays, February 1999-January 2000Continued

RUM BAY-CONTINUED

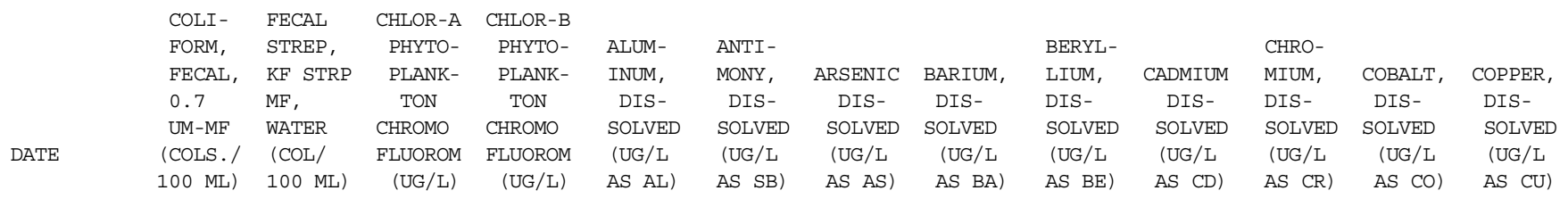

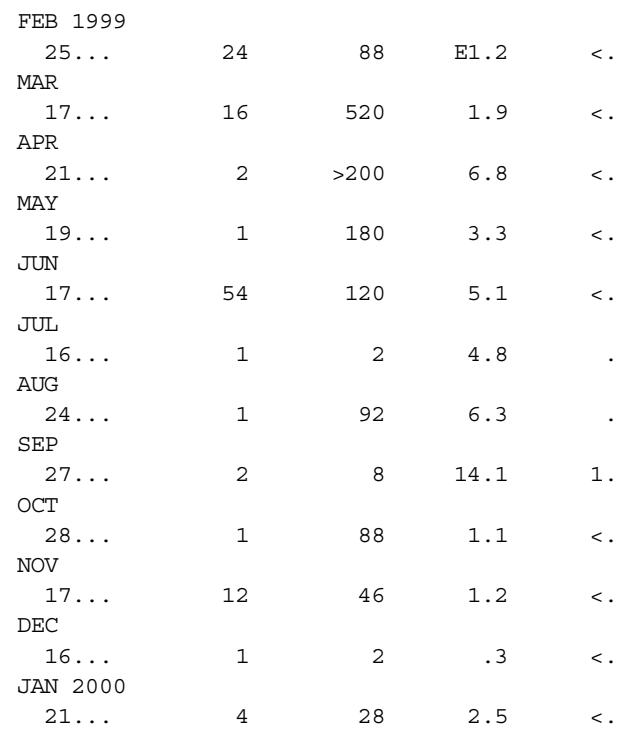

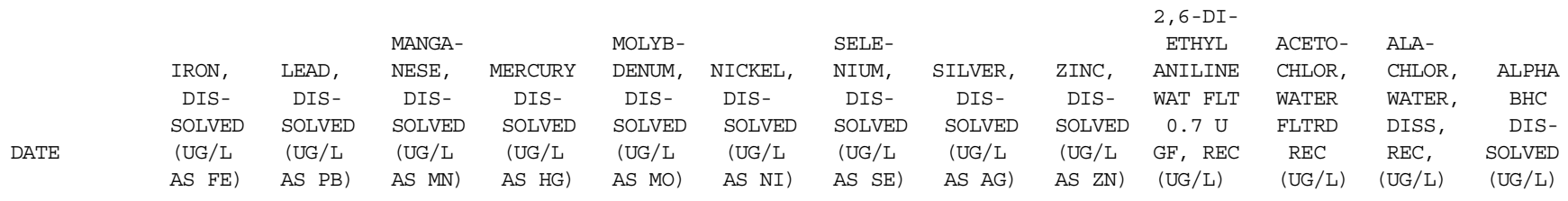

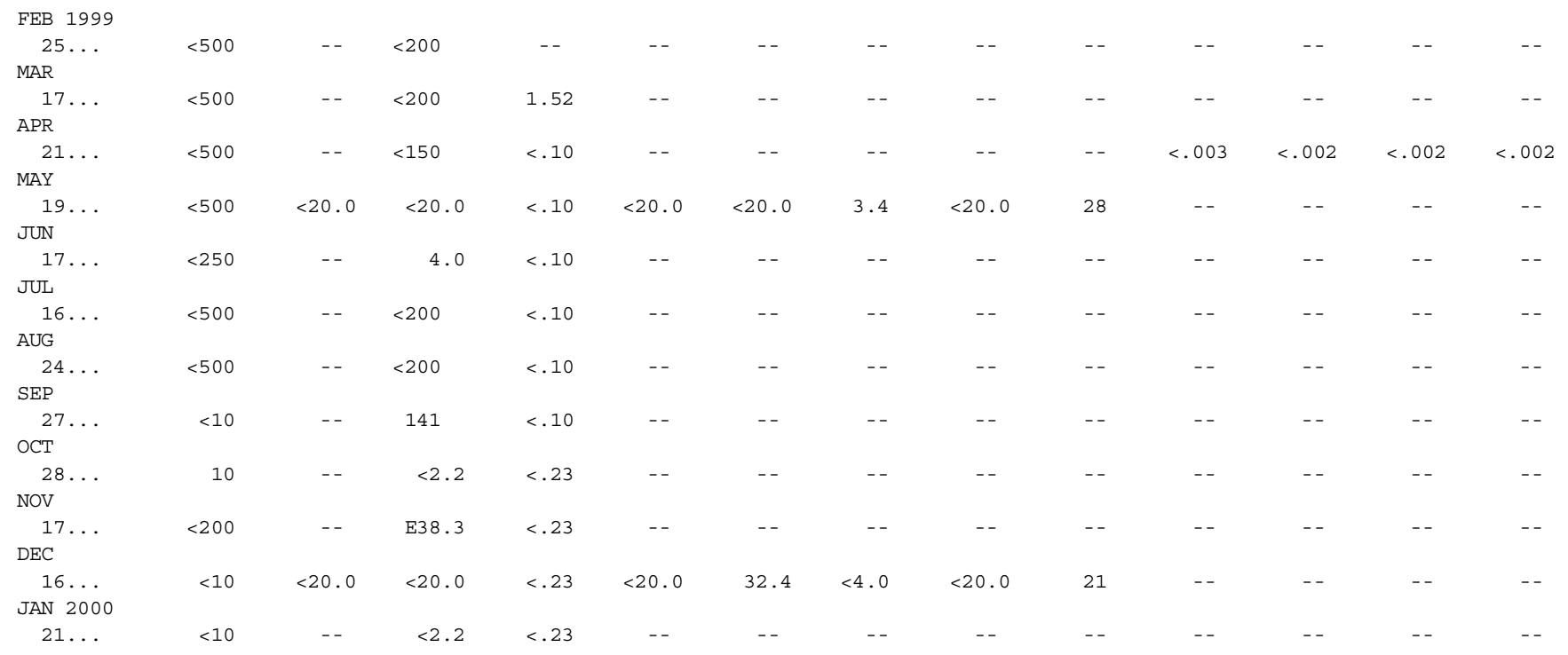


Table 4. Monthly and periodic water-quality data in Christmas, Bastrop, and Drum Bays, February 1999-January 2000Continued

\begin{tabular}{|c|c|c|c|c|c|c|c|c|c|c|c|c|c|}
\hline \multicolumn{14}{|c|}{ DRUM BAY—CONTINUED } \\
\hline TE & $\begin{array}{l}\text { ATRA- } \\
\text { ZINE, } \\
\text { WATER, } \\
\text { DISS, } \\
\text { REC } \\
\text { (UG/L) }\end{array}$ & $\begin{array}{c}\text { BEN- } \\
\text { FLUR- } \\
\text { ALIN } \\
\text { WAT FLD } \\
0.7 \text { U } \\
\text { GF, REC } \\
\text { (UG/L) }\end{array}$ & $\begin{array}{l}\text { BUTYL- } \\
\text { ATE, } \\
\text { WATER, } \\
\text { DISS, } \\
\text { REC } \\
\text { (UG/L) }\end{array}$ & $\begin{array}{l}\text { CAR- } \\
\text { BARYL } \\
\text { WATER } \\
\text { FLTRD } \\
0.7 \text { U } \\
\text { GF, REC } \\
\text { (UG/L) }\end{array}$ & $\begin{array}{l}\text { CARBO- } \\
\text { FURAN } \\
\text { WATER } \\
\text { FLTRD } \\
0.7 \mathrm{U} \\
\text { GF, REC } \\
\text { (UG/L) }\end{array}$ & $\begin{array}{c}\text { CHLOR- } \\
\text { PYRIFOS } \\
\text { DIS- } \\
\text { SOLVED } \\
\text { (UG/L) }\end{array}$ & $\begin{array}{l}\text { CYANA- } \\
\text { ZINE, } \\
\text { WATER, } \\
\text { DISS, } \\
\text { REC } \\
\text { (UG/L) }\end{array}$ & $\begin{array}{l}\text { DCPA } \\
\text { WATER } \\
\text { FLTRD } \\
0.7 \mathrm{U} \\
\text { GF, REC } \\
\text { (UG/L) }\end{array}$ & $\begin{array}{l}\text { DEETHYL } \\
\text { ATRA- } \\
\text { ZINE, } \\
\text { WATER, } \\
\text { DISS, } \\
\text { REC } \\
\text { (UG/L) }\end{array}$ & $\begin{array}{l}\text { DIAZ- } \\
\text { INON } \\
\text { D10 SRG } \\
\text { WAT FLT } \\
0.7 \quad \text { U } \\
\text { GF, REC } \\
\text { PERCENT }\end{array}$ & $\begin{array}{c}\text { DI- } \\
\text { AZINON, } \\
\text { DIS- } \\
\text { SOLVED } \\
\text { (UG/L) }\end{array}$ & $\begin{array}{l}\text { DI- } \\
\text { ELDRIN } \\
\text { DIS- } \\
\text { SOLVED } \\
\text { (UG/L) }\end{array}$ & $\begin{array}{l}\text { DISUL- } \\
\text { FOTON } \\
\text { WATER } \\
\text { FLTRD } \\
0.7 \text { U } \\
\text { GF, REC } \\
\text { (UG/L) }\end{array}$ \\
\hline
\end{tabular}

FEB 1999
$25 \ldots$
MAR
$17 \ldots$
APR
$21 \ldots$
MAY
$19 \ldots$
JUN
$17 \ldots$
JUL
$16 \ldots$
AUG
$24 \ldots$
SEP
$27 \ldots$
OCT
$28 \ldots$
NOV
$17 \ldots$
DEC
$16 \ldots$
JAN 2000
$21 \ldots$

\begin{tabular}{|c|c|c|c|c|c|c|c|c|c|c|c|c|c|}
\hline & $\begin{array}{l}\text { EPTC } \\
\text { WATER }\end{array}$ & $\begin{array}{l}\text { ETHAL- } \\
\text { FLUR- } \\
\text { ALIN }\end{array}$ & $\begin{array}{l}\text { ETHO- } \\
\text { PROP } \\
\text { WATER }\end{array}$ & FONOFOS & $\begin{array}{c}\text { HCH } \\
\text { ALPHA } \\
\text { D6 SRG }\end{array}$ & & $\begin{array}{l}\text { LIN- } \\
\text { URON } \\
\text { WATER }\end{array}$ & MALA- & $\begin{array}{c}\text { METHYL } \\
\text { AZIN- } \\
\text { PHOS }\end{array}$ & $\begin{array}{c}\text { METHYL } \\
\text { PARA- } \\
\text { THION }\end{array}$ & METO- & $\begin{array}{l}\text { METRI- } \\
\text { BUZIN }\end{array}$ & $\begin{array}{l}\text { MOL- } \\
\text { INATE } \\
\text { WATER }\end{array}$ \\
\hline & FLTRD & WAT FLT & FLTRD & WATER & WAT FLT & LINDANE & FLTRD & THION, & WAT FLT & WAT FLT & LACHLOR & SENCOR & FLTRD \\
\hline & $0.7 \mathrm{U}$ & $0.7 \mathrm{U}$ & $0.7 \mathrm{U}$ & DISS & $0.7 \mathrm{U}$ & DIS- & $0.7 \mathrm{U}$ & DIS- & $0.7 \mathrm{U}$ & $0.7 \mathrm{U}$ & WATER & WATER & $0.7 \mathrm{U}$ \\
\hline DATE & $\begin{array}{l}\text { GF, REC } \\
\text { (UG/L) }\end{array}$ & $\begin{array}{l}\text { GF， REC } \\
\text { (UG/L) }\end{array}$ & $\begin{array}{l}\text { GF， REC } \\
\text { (UG/L) }\end{array}$ & $\begin{array}{c}\mathrm{REC} \\
\text { (UG/L) }\end{array}$ & $\begin{array}{l}\text { GF， REC } \\
\text { PERCENT }\end{array}$ & $\begin{array}{l}\text { SOLVED } \\
\text { (UG/L) }\end{array}$ & $\begin{array}{l}\text { GF， REC } \\
\text { (UG/L) }\end{array}$ & $\begin{array}{l}\text { SOLVED } \\
\text { (UG/L) }\end{array}$ & $\begin{array}{l}\text { GF, REC } \\
\text { (UG/L) }\end{array}$ & $\begin{array}{l}\text { GF， REC } \\
\text { (UG/L) }\end{array}$ & $\begin{array}{l}\text { DISSOLV } \\
\text { (UG/L) }\end{array}$ & $\begin{array}{l}\text { DISSOLV } \\
\text { (UG/L) }\end{array}$ & $\begin{array}{l}\text { GF, REC } \\
\text { (UG/L) }\end{array}$ \\
\hline
\end{tabular}

FEB 1999

$25 \ldots$
MAR
$17 \ldots$
APR
$21 \ldots$
MAY
$19 \ldots$
JUN
$17 \ldots$
JUL
$16 \ldots$
AUG
$24 \ldots$
SEP
$27 \ldots$
OCT
$28 \ldots$
NOV
$17 \ldots$
DEC
$16 \ldots$
JAN 2000
$21 .$.

$.100<.002<.002<.003<.003<.004 \quad<.010<.002 \quad$ E. $012 \quad 75 \quad<.002 \quad<.001<.017$

$--$


Table 4. Monthly and periodic water-quality data in Christmas, Bastrop, and Drum Bays, February 1999-January 2000Continued

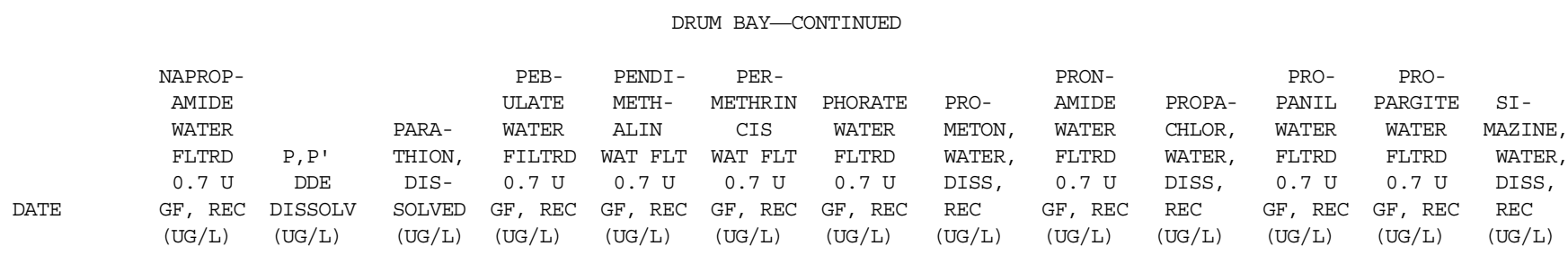

FEB 1999
$25 \ldots$
MAR $\ldots$
$17 \ldots$
APR
$21 \ldots$
MAY $\ldots$
$19 \ldots$
JUN
$17 \ldots$
JUL
$16 \ldots$
AUG
$24 \ldots$
SEP
$27 \ldots$
OCT
$28 \ldots$
NOV
$17 \ldots$
DEC
$16 \ldots$
JAN 2000
$21 .$.

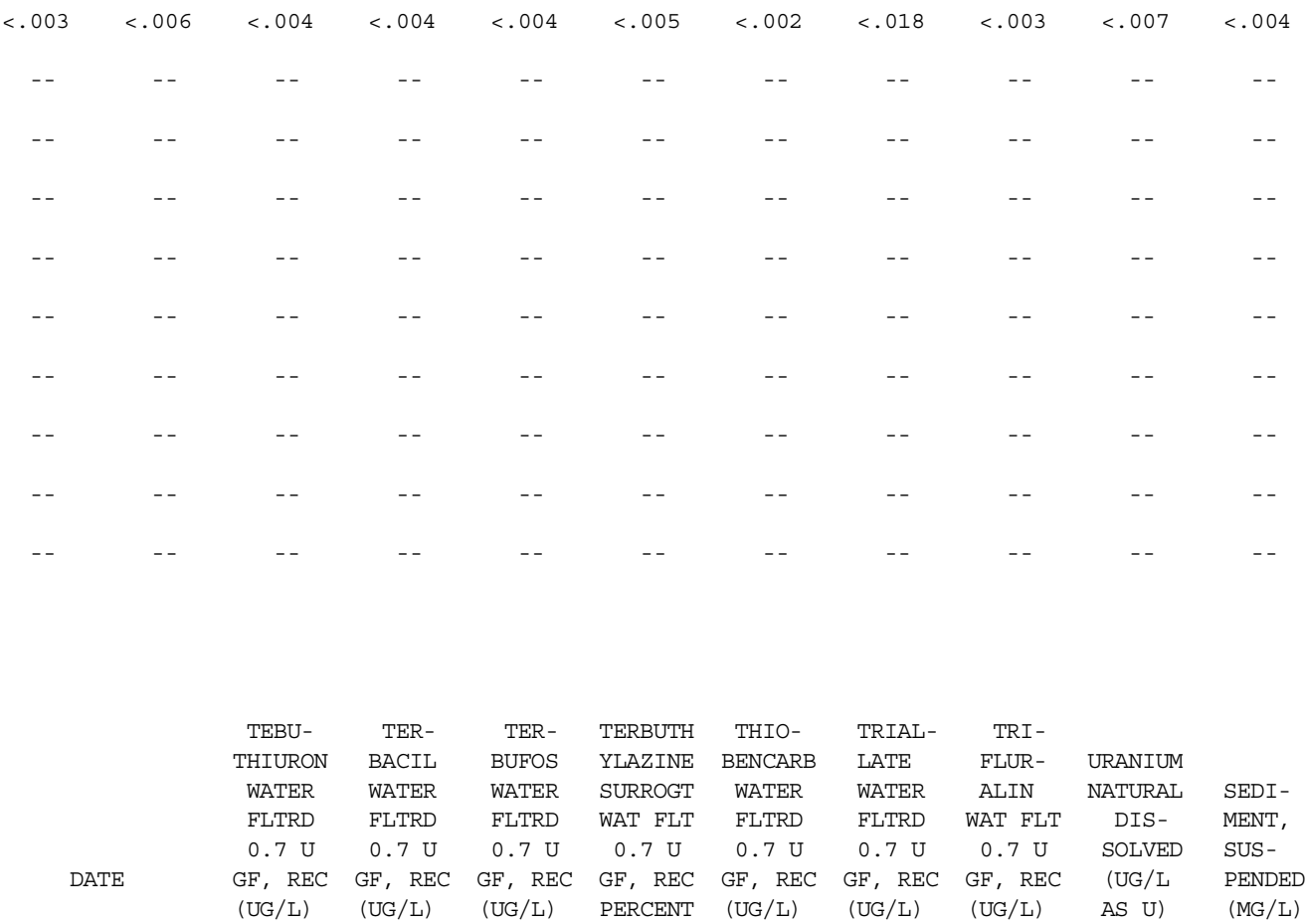

\begin{tabular}{|c|c|c|c|c|c|c|c|c|c|}
\hline FEB 1999 & & & & & & & & & \\
\hline $25 \ldots$ & -- & -- & -- & -- & -- & -- & -- & -- & 47 \\
\hline MAR & & & & & & & & & \\
\hline $17 \ldots$ & -- & -- & -- & -- & -- & -- & -- & -- & 106 \\
\hline APR & & & & & & & & & \\
\hline $21 \ldots$ & $<.010$ & $<.007$ & $<.013$ & 112 & $<.002$ & $<.001$ & $<.002$ & -- & 478 \\
\hline MAY & & & & & & & & & \\
\hline $19 \ldots$ & -- & -- & -- & -- & -- & -- & -- & $<20.0$ & 27 \\
\hline JUN & & & & & & & & & \\
\hline $17 \ldots$ & -- & -- & -- & -- & -- & -- & -- & -- & 49 \\
\hline JUL & & & & & & & & & \\
\hline $16 \ldots$ & -- & -- & -- & -- & -- & -- & -- & -- & 37 \\
\hline AUG & & & & & & & & & \\
\hline $24 \ldots$ & -- & -- & -- & -- & -- & -- & -- & -- & 36 \\
\hline SEP & & & & & & & & & \\
\hline $27 \ldots$ & -- & -- & -- & -- & -- & -- & -- & -- & 18 \\
\hline ОСТ & & & & & & & & & \\
\hline $28 \ldots$ & -- & -- & -- & -- & -- & -- & -- & -- & 34 \\
\hline NOV & & & & & & & & & \\
\hline $17 \ldots$ & -- & -- & -- & -- & -- & -- & -- & -- & 69 \\
\hline DEC & & & & & & & & & \\
\hline $16 \ldots$ & -- & -- & -- & -- & -- & -- & -- & $<20.0$ & 9 \\
\hline JAN 2000 & & & & & & & & & \\
\hline $21 \ldots$ & -- & -- & -- & -- & -- & $\ldots$ & $\ldots$ & $\ldots$ & 165 \\
\hline
\end{tabular}


Table 5. Semivolatile organic compounds in samples collected using a semipermeable membrane device deployed in Christmas Bay, February 29-March 29, 2000

$[\mu \mathrm{g} / \mathrm{kg}$, micrograms per kilogram; <, less than]

\begin{tabular}{|c|c|c|c|c|}
\hline \multirow{2}{*}{ Compound } & \multicolumn{3}{|c|}{$\begin{array}{c}\text { Concentration } \\
(\mu \mathbf{g} / \mathbf{k g})\end{array}$} & \multirow{2}{*}{$\begin{array}{c}\begin{array}{c}\text { Recovery } \\
\text { (percent) }\end{array} \\
\text { Laboratory matrix spike }\end{array}$} \\
\hline & Environmental sample & Field blank & Laboratory blank & \\
\hline Phenol & $1,228.0$ & $1_{39.1}$ & $<50$ & 78.56 \\
\hline bis(2-Chloroethyl)ether & $<100$ & $<100$ & $<50$ & 77.99 \\
\hline 2-Chlorophenol & $<100$ & $<100$ & $<50$ & 80.65 \\
\hline 1,3-Dichlorobenzene & $<100$ & $<100$ & $<50$ & 71.48 \\
\hline 1,4-Dichlorobenzene & $<100$ & $<100$ & $<50$ & 77.35 \\
\hline 1,2-Dichlorobenzene & $<100$ & $<100$ & $<50$ & 76.43 \\
\hline bis(2-Chloroisopropyl)ether & $<100$ & $<100$ & $<50$ & 80.50 \\
\hline Hexachloroethane & $<100$ & $<100$ & $<50$ & 76.32 \\
\hline$N$-Nitrosodi- $n$-propylamine & $<100$ & $<100$ & $<50$ & 75.96 \\
\hline$p$-Cresol & ${ }^{1} 3.9$ & $<100$ & $<50$ & 77.84 \\
\hline Nitrobenzene & $<100$ & $<100$ & $<50$ & 75.50 \\
\hline Isophorone & $<100$ & $<100$ & $<50$ & 77.49 \\
\hline 2-Nitrophenol & $<100$ & $<100$ & $<50$ & 82.39 \\
\hline C8-Alkylphenol & $<100$ & $<100$ & $<50$ & 69.67 \\
\hline bis(2-Chloroethoxy)methane & $<100$ & $<100$ & $<50$ & 77.48 \\
\hline 2,4-Dichlorophenol & $<100$ & $<100$ & $<50$ & 77.96 \\
\hline 3,5-Dimethylphenol & $<100$ & $<100$ & $<50$ & 69.97 \\
\hline 1,2,4-Trichlorobenzene & $<100$ & $<100$ & $<50$ & 80.33 \\
\hline Naphthalene & $<100$ & $<100$ & $<50$ & 85.01 \\
\hline 2,4,6-Trimethylphenol & $<100$ & $<100$ & $<50$ & ${ }^{1} 18.89$ \\
\hline Hexachlorobutadiene & $<100$ & $<100$ & $<50$ & 79.86 \\
\hline Quinoline & $<100$ & $<100$ & $<50$ & 83.57 \\
\hline Isoquinoline & $<100$ & $<100$ & $<50$ & 72.28 \\
\hline 4-Chloro-3-methylphenol & ${ }^{1} 5.1$ & $<100$ & $<50$ & 79.70 \\
\hline Hexachlorocyclopentadiene & $<100$ & $<100$ & $<50$ & $1_{39.47}$ \\
\hline 2,4,6-Trichlorophenol & $<100$ & $<100$ & $<50$ & 74.56 \\
\hline 2-Chloronaphthalene & $<100$ & $<100$ & $<50$ & 79.23 \\
\hline 2-Ethylnaphthalene & $<100$ & $<100$ & $<50$ & 72.79 \\
\hline 2,6-Dimethylnaphthalene & ${ }^{1} 3.1$ & $<100$ & $<50$ & 84.32 \\
\hline 1,6-Dimethylnaphthalene & ${ }^{1} 1.0$ & $<100$ & $<50$ & 80.83 \\
\hline Acenaphthylene & $<100$ & $<100$ & $<50$ & 78.86 \\
\hline 1,2-Dimethylnaphthalene & $<100$ & $<100$ & $<50$ & 77.50 \\
\hline Dimethylphthalate & $<100$ & $<100$ & $<50$ & 81.50 \\
\hline 2,6-Dinitrotoluene & $<100$ & $<100$ & $<50$ & 81.50 \\
\hline Acenaphthene & $<100$ & $<100$ & $<50$ & 83.89 \\
\hline 4-Nitrophenol & $<100$ & $<100$ & $<50$ & 0 \\
\hline 2,4-Dinitrotoluene & $<100$ & $<100$ & $<50$ & 88.87 \\
\hline 2,4-Dinitrophenol & $<100$ & $<100$ & $<50$ & 90.54 \\
\hline 2,3,6-Trimethylnaphthalene & $<100$ & $<100$ & $<50$ & 81.99 \\
\hline 9H-Fluorene & $<100$ & $<100$ & $<50$ & 86.90 \\
\hline 4-Chlorophenyl-phenylether & $<100$ & $<100$ & $<50$ & 83.54 \\
\hline Diethylphthalate & $<100$ & ${ }^{1} 17.6$ & $<50$ & 92.45 \\
\hline $\mathrm{N}$-Nitrosodiphenylamine & $<100$ & $<100$ & $<50$ & 92.31 \\
\hline Azobenzene & $<100$ & $<100$ & $<50$ & 81.33 \\
\hline
\end{tabular}

Footnotes at end of table. 
Table 5. Semivolatile organic compounds in samples collected using a semipermeable membrane device deployed in Christmas Bay, February 29-March 29, 2000-Continued

\begin{tabular}{|c|c|c|c|c|}
\hline \multirow[t]{2}{*}{ Compound } & \multicolumn{3}{|c|}{$\begin{array}{c}\text { Concentration } \\
(\mu \mathbf{g} / \mathbf{k g})\end{array}$} & \multirow{2}{*}{$\begin{array}{c}\begin{array}{c}\text { Recovery } \\
\text { (percent) }\end{array} \\
\text { Laboratory matrix spike }\end{array}$} \\
\hline & Environmental sample & Field blank & Laboratory blank & \\
\hline 4-Bromophenyl-phenylether & $<100$ & $<100$ & $<50$ & 86.04 \\
\hline 1-Methyl-9H-fluorene & $<100$ & $<100$ & $<50$ & 92.05 \\
\hline Hexachlorobenzene & $<100$ & $<100$ & $<50$ & 86.67 \\
\hline Pentachloroanisole & $<100$ & $<100$ & $<50$ & 87.97 \\
\hline Dibenzothiophene & $<100$ & $<100$ & $<50$ & 87.00 \\
\hline Pentachlorophenol & $<100$ & $<100$ & $<50$ & 94.82 \\
\hline Pentachloronitrobenzene & $<100$ & $<100$ & $<50$ & 86.40 \\
\hline Phenanthrene & $<100$ & $<100$ & $<50$ & 87.27 \\
\hline Anthracene & $<100$ & $<100$ & $<50$ & 85.13 \\
\hline Acridine & $<100$ & $<100$ & $<50$ & 91.48 \\
\hline Phenanthridine & $<100$ & $<100$ & $<50$ & 92.91 \\
\hline 9H-Carbazol & $<100$ & $<100$ & $<50$ & 90.58 \\
\hline 2-Methylanthracene & $<100$ & $<100$ & $<50$ & 86.96 \\
\hline Benzo $[c]$ cinnoline & $<100$ & $<100$ & $<50$ & 90.14 \\
\hline 4,5-Methylenephenanthrene & $<100$ & $<100$ & $<50$ & 86.17 \\
\hline 1-Methylphenanthrene & $<100$ & $<100$ & $<50$ & 88.46 \\
\hline Di- $n$-butylphthalate & $<100$ & ${ }^{1} 12.8$ & $<50$ & 93.63 \\
\hline Anthraquinone & $<100$ & $<100$ & $<50$ & 82.35 \\
\hline Fluoranthene & $<100$ & $<100$ & $<50$ & 93.35 \\
\hline Pyrene & $<100$ & $<100$ & $<50$ & 84.84 \\
\hline 1-Methylpyrene & $<100$ & $<100$ & $<50$ & 80.34 \\
\hline Butylbenzylphthalate & $<100$ & $<100$ & $<50$ & 87.67 \\
\hline Benz $[a]$ anthracene & $<100$ & $<100$ & $<50$ & 89.98 \\
\hline Chrysene & $<100$ & $<100$ & $<50$ & 92.17 \\
\hline bis(2-Ethylhexyl)phthalate & ${ }^{2} 105$ & 198 & $<50$ & 93.16 \\
\hline 2,2-Biquinoline & ${ }^{1} 3.5$ & $<100$ & $<50$ & 93.09 \\
\hline Di- $n$-octylphthalate & 15.4 & $<100$ & $<50$ & 93.97 \\
\hline Benzo $[b]$ fluoranthene & ${ }^{1} 3.0$ & $<100$ & $<50$ & 93.01 \\
\hline Benzo $[k]$ fluoranthene & ${ }^{1} .73$ & $<100$ & $<50$ & 85.42 \\
\hline Benzo $[a]$ pyrene & $<100$ & $<100$ & $<50$ & 90.99 \\
\hline Indeno[1,2,3-cd]pyrene & $<100$ & $<100$ & $<50$ & 97.83 \\
\hline Dibenz $[a, h]$ anthracene & $<100$ & $<100$ & $<50$ & 89.93 \\
\hline Benzo $[g h i]$ perylene & $<100$ & $<100$ & $<50$ & 92.37 \\
\hline
\end{tabular}

${ }^{1}$ Estimated.

2 Contaminated.

Table 6. Grain size in bed-sediment samples collected in Christmas, Bastrop, and Drum Bays, November 30, 1999

[Size of sieve was 0.062 millimeter; size of pipet was 0.004 millimeter. QA, quality assurance]

\begin{tabular}{lcc}
\hline \multicolumn{1}{c}{ Station name } & $\begin{array}{c}\text { Sieve analysis } \\
\text { (percent silt and clay) }\end{array}$ & $\begin{array}{c}\text { Pipet analysis } \\
\text { (percent clay) }\end{array}$ \\
\hline Christmas Bay & 32.5 & 21.2 \\
Bastrop Bay & 57.4 & 26.9 \\
Drum Bay & 72.5 & 44.7 \\
Drum Bay split (QA) & 72.1 & 45.9 \\
\hline
\end{tabular}


Table 7. Carbon and major and trace elements in bed-sediment samples collected in Christmas, Bastrop, and Drum Bays, November 30, 1999

[UG/G, micrograms per gram; <, less than]

\begin{tabular}{|c|c|c|c|c|c|c|c|c|c|c|c|c|c|}
\hline & & & & & & CHRISTM & BAY & & & & & & \\
\hline DATE & STATION & NUMBER & DATE & TIME & $\begin{array}{c}\text { CALCIUM } \\
\text { BOT MAT } \\
\text { FIELD } \\
\text { PERCENT }\end{array}$ & $\begin{array}{l}\text { MAGNE- } \\
\text { SIUM } \\
\text { BOT MAT } \\
\text { FIELD } \\
\text { PERCENT }\end{array}$ & $\begin{array}{l}\text { POTAS- } \\
\text { SIUM } \\
\text { BOT MAT } \\
\text { FIELD } \\
\text { PERCENT }\end{array}$ & $\begin{array}{l}\text { SODIUM } \\
\text { BOT MAT } \\
\text { FIELD } \\
\text { PERCENT }\end{array}$ & $\begin{array}{l}\text { SULFUR } \\
\text { BOT MAT } \\
\text { FIELD } \\
\text { PERCENT }\end{array}$ & $\begin{array}{c}\text { PHOS- } \\
\text { PHORUS } \\
\text { BOT MAT } \\
\text { FIELD } \\
\text { PERCENT }\end{array}$ & $\begin{array}{c}\text { CARBON, } \\
\text { INORG, } \\
\text { SED, BM } \\
\text { WS, < }<63 \mathrm{U} \\
\text { (PER- } \\
\text { CENT) }\end{array}$ & $\begin{array}{c}\text { CARBON, } \\
\text { ORG + } \\
\text { INORG, } \\
\text { SED, BM } \\
\text { DW, REC } \\
\text { PERCENT }\end{array}$ & $\begin{array}{c}\text { CARBON, } \\
\text { ORGANIC } \\
\text { SED, BM } \\
\text { WS, <63U } \\
\text { (PER- } \\
\text { CENT) }\end{array}$ \\
\hline NOV & & & & & & & & & & & & & \\
\hline $30 \ldots$ & 08078800 & & 19991130 & 1149 & 1.1 & 1.7 & 2.3 & 2.5 & .29 & .059 & .28 & 1.2 & .91 \\
\hline & $\begin{array}{l}\text { ALUM- } \\
\text { INUM }\end{array}$ & $\begin{array}{l}\text { ANTI - } \\
\text { MONY }\end{array}$ & ARSENIC & BARIUM & $\begin{array}{l}\text { BERYL- } \\
\text { LIUM }\end{array}$ & BISMUTH & CADMIUM & CERIUM & $\begin{array}{l}\text { CHRO- } \\
\text { MIUM }\end{array}$ & COBALT & COPPER & $\begin{array}{l}\text { EURO- } \\
\text { PIUM }\end{array}$ & GALLIUM \\
\hline DATE & $\begin{array}{c}\text { BOT MAT } \\
\text { FIELD } \\
\text { PERCENT }\end{array}$ & $\begin{array}{c}\text { BOT MAT } \\
\text { FIELD } \\
\text { (UG/G) }\end{array}$ & $\begin{array}{c}\text { BOT MAT } \\
\text { FIELD } \\
\text { (UG/G) }\end{array}$ & $\begin{array}{l}\text { BOT MAT } \\
\text { FIELD } \\
\text { (UG/G) }\end{array}$ & $\begin{array}{l}\text { BOT MAT } \\
\text { FIELD } \\
\text { (UG/G) }\end{array}$ & $\begin{array}{l}\text { BOT MAT } \\
\text { FIELD } \\
\text { (UG/G) }\end{array}$ & $\begin{array}{l}\text { BOT MAT } \\
\text { FIELD } \\
\text { (UG/G) }\end{array}$ & $\begin{array}{l}\text { BOT MAT } \\
\text { FIELD } \\
\text { (UG/G) }\end{array}$ & $\begin{array}{l}\text { BOT MAT } \\
\text { FIELD } \\
\text { (UG/G) }\end{array}$ & $\begin{array}{c}\text { BOT MAT } \\
\text { FIELD } \\
\text { (UG/G) }\end{array}$ & $\begin{array}{c}\text { BOT MAT } \\
\text { FIELD } \\
\text { (UG/G) }\end{array}$ & $\begin{array}{c}\text { BOT MAT } \\
\text { FIELD } \\
\text { (UG/G) }\end{array}$ & $\begin{array}{c}\text { BOT MAT } \\
\text { FIELD } \\
\text { (UG/G) }\end{array}$ \\
\hline NOV & & & & & & & & & & & & & \\
\hline $30 \ldots$ & 7.6 & .7 & 7.3 & 430 & 2.0 & $<1$ & $<.1$ & 74 & 76 & 12 & 22 & 1 & 17 \\
\hline & GOLD & HOLMIUM & IRON & $\begin{array}{c}\text { LANTHA- } \\
\text { NUM }\end{array}$ & LEAD & LITHIUM & $\begin{array}{l}\text { MANGA- } \\
\text { NESE }\end{array}$ & MERCURY & $\begin{array}{l}\text { MOLYB- } \\
\text { DENUM }\end{array}$ & $\begin{array}{c}\text { NEODYM- } \\
\text { IUM }\end{array}$ & NICKEL & NIOBIUM & $\begin{array}{l}\text { SCAN- } \\
\text { DIUM }\end{array}$ \\
\hline DATE & $\begin{array}{c}\text { BOT MAT } \\
\text { FIELD } \\
\text { (UG/G) }\end{array}$ & $\begin{array}{c}\text { BOT MAT } \\
\text { FIELD } \\
\text { (UG/G) }\end{array}$ & $\begin{array}{l}\text { BOT MAT } \\
\text { FIELD } \\
\text { PERCENT }\end{array}$ & $\begin{array}{c}\text { BOT MAT } \\
\text { FIELD } \\
\text { (UG/G) }\end{array}$ & $\begin{array}{c}\text { BOT MAT } \\
\text { FIELD } \\
\text { (UG/G) }\end{array}$ & $\begin{array}{c}\text { BOT MAT } \\
\text { FIELD } \\
\text { (UG/G) }\end{array}$ & $\begin{array}{c}\text { BOT MAT } \\
\text { FIELD } \\
\text { (UG/G) }\end{array}$ & $\begin{array}{c}\text { BOT MAT } \\
\text { FIELD } \\
\text { (UG/G) }\end{array}$ & $\begin{array}{c}\text { BOT MAT } \\
\text { FIELD } \\
\text { (UG/G) }\end{array}$ & $\begin{array}{c}\text { BOT MAT } \\
\text { FIELD } \\
\text { (UG/G) }\end{array}$ & $\begin{array}{c}\text { BOT MAT } \\
\text { FIELD } \\
\text { (UG/G) }\end{array}$ & $\begin{array}{c}\text { BOT MAT } \\
\text { FIELD } \\
\text { (UG/G) }\end{array}$ & $\begin{array}{c}\text { BOT MAT } \\
\text { FIELD } \\
\text { (UG/G) }\end{array}$ \\
\hline NOV & & & & & & & & & & & & & \\
\hline $30 \ldots$ & $<1$ & 1 & 3.6 & 44 & 24 & 53 & 540 & .03 & .6 & 34 & 28 & 14 & 13 \\
\hline & $\begin{array}{l}\text { SELE- } \\
\text { NIUM }\end{array}$ & SILVER & $\begin{array}{l}\text { STRON- } \\
\text { TIUM }\end{array}$ & $\begin{array}{l}\text { TANTA- } \\
\text { LUM }\end{array}$ & $\begin{array}{l}\text { THAL- } \\
\text { LIUM } \\
\text { BED MAT }\end{array}$ & TIN & $\begin{array}{l}\text { TITA- } \\
\text { NIUM, } \\
\text { SED， BM }\end{array}$ & $\begin{array}{l}\text { VANA- } \\
\text { DIUM }\end{array}$ & $\begin{array}{l}\text { YTTER- } \\
\text { BIUM }\end{array}$ & YTTRIUM & ZINC & THORIUM & URANIUM \\
\hline DATE & $\begin{array}{c}\text { BOT MAT } \\
\text { FIELD } \\
\text { (UG/G) }\end{array}$ & $\begin{array}{c}\text { BOT MAT } \\
\text { FIELD } \\
\text { (UG/G) }\end{array}$ & $\begin{array}{l}\text { BOT MAT } \\
\text { FIELD } \\
\text { (UG/G) }\end{array}$ & $\begin{array}{l}\text { BOT MAT } \\
\text { FIELD } \\
\text { (UG/G) }\end{array}$ & $\begin{array}{c}\text { D SIEVE } \\
\text { TOTAL } \\
\text { (UG/G) }\end{array}$ & $\begin{array}{c}\text { BOT MAT } \\
\text { FIELD } \\
\text { (UG/G) }\end{array}$ & $\begin{array}{c}\text { WS },<63 \mathrm{U} \\
\text { REC } \\
\text { PERCENT }\end{array}$ & $\begin{array}{l}\text { BOT MAT } \\
\text { FIELD } \\
\text { (UG/G) }\end{array}$ & $\begin{array}{c}\text { BOT MAT } \\
\text { FIELD } \\
\text { (UG/G) }\end{array}$ & $\begin{array}{c}\text { BOT MAT } \\
\text { FIELD } \\
\text { (UG/G) }\end{array}$ & $\begin{array}{c}\text { BOT MAT } \\
\text { FIELD } \\
\text { (UG/G) }\end{array}$ & $\begin{array}{c}\text { BOT MAT } \\
\text { FIELD } \\
\text { (UG/G) }\end{array}$ & $\begin{array}{c}\text { BOT MAT } \\
\text { FIELD } \\
\text { (UG/G) }\end{array}$ \\
\hline
\end{tabular}

Nov

$\begin{array}{lllllllllllllll}30 & . & .3 & .5 & 120 & 1 & <1 & 3 & .390 & 110 & 2 & 24 & 100 & 13 & 3.1\end{array}$

\begin{tabular}{|c|c|c|c|c|c|c|c|c|c|c|c|c|c|}
\hline & & & & & & BASTROP & $\mathrm{BAY}$ & & & & & & \\
\hline DATE & STATION & NUMBER & DATE & TIME & $\begin{array}{c}\text { CALCIUM } \\
\text { BOT MAT } \\
\text { FIELD } \\
\text { PERCENT }\end{array}$ & $\begin{array}{l}\text { MAGNE- } \\
\text { SIUM } \\
\text { BOT MAT } \\
\text { FIELD } \\
\text { PERCENT }\end{array}$ & $\begin{array}{l}\text { POTAS- } \\
\text { SIUM } \\
\text { BOT MAT } \\
\text { FIELD } \\
\text { PERCENT }\end{array}$ & $\begin{array}{l}\text { SODIUM } \\
\text { BOT MAT } \\
\text { FIELD } \\
\text { PERCENT }\end{array}$ & $\begin{array}{l}\text { SULFUR } \\
\text { BOT MAT } \\
\text { FIELD } \\
\text { PERCENT }\end{array}$ & $\begin{array}{c}\text { PHOS- } \\
\text { PHORUS } \\
\text { BOT MAT } \\
\text { FIELD } \\
\text { PERCENT }\end{array}$ & $\begin{array}{c}\text { CARBON, } \\
\text { INORG, } \\
\text { SED, BM } \\
\text { WS, < }<63 \mathrm{U} \\
\text { (PER- } \\
\text { CENT) }\end{array}$ & $\begin{array}{c}\text { CARBON, } \\
\text { ORG + } \\
\text { INORG, } \\
\text { SED, BM } \\
\text { DW, REC } \\
\text { PERCENT }\end{array}$ & $\begin{array}{c}\text { CARBON, } \\
\text { ORGANIC } \\
\text { SED, BM } \\
\text { WS, <63U } \\
\text { (PER- } \\
\text { CENT) }\end{array}$ \\
\hline JOV & & & & & & & & & & & & & \\
\hline $30 \ldots$ & 29055609 & 5103501 & 19991130 & 1055 & 1.2 & 1.4 & 2.0 & 2.7 & .44 & .055 & .28 & 1.2 & .89 \\
\hline & $\begin{array}{l}\text { ALUM- } \\
\text { INUM }\end{array}$ & $\begin{array}{l}\text { ANTI- } \\
\text { MONY }\end{array}$ & ARSENIC & BARIUM & $\begin{array}{l}\text { BERYL- } \\
\text { LIUM }\end{array}$ & BISMUTH & CADMIUM & CERIUM & $\begin{array}{l}\text { CHRO- } \\
\text { MIUM }\end{array}$ & COBALT & COPPER & $\begin{array}{l}\text { EURO- } \\
\text { PIUM }\end{array}$ & GALLIUM \\
\hline DATE & $\begin{array}{c}\text { BOT MAT } \\
\text { FIELD } \\
\text { PERCENT }\end{array}$ & $\begin{array}{l}\text { BOT MAT } \\
\text { FIELD } \\
\text { (UG/G) }\end{array}$ & $\begin{array}{c}\text { BOT MAT } \\
\text { FIELD } \\
\text { (UG/G) }\end{array}$ & $\begin{array}{c}\text { BOT MAT } \\
\text { FIELD } \\
\text { (UG/G) }\end{array}$ & $\begin{array}{c}\text { BOT MAT } \\
\text { FIELD } \\
\text { (UG/G) }\end{array}$ & $\begin{array}{c}\text { BOT MAT } \\
\text { FIELD } \\
\text { (UG/G) }\end{array}$ & $\begin{array}{c}\text { BOT MAT } \\
\text { FIELD } \\
\text { (UG/G) }\end{array}$ & $\begin{array}{c}\text { BOT MAT } \\
\text { FIELD } \\
\text { (UG/G) }\end{array}$ & $\begin{array}{c}\text { BOT MAT } \\
\text { FIELD } \\
\text { (UG/G) }\end{array}$ & $\begin{array}{c}\text { BOT MAT } \\
\text { FIELD } \\
\text { (UG/G) }\end{array}$ & $\begin{array}{c}\text { BOT MAT } \\
\text { FIELD } \\
\text { (UG/G) }\end{array}$ & $\begin{array}{c}\text { BOT MAT } \\
\text { FIELD } \\
\text { (UG/G) }\end{array}$ & $\begin{array}{c}\text { BOT MAT } \\
\text { FIELD } \\
\text { (UG/G) }\end{array}$ \\
\hline
\end{tabular}

NOV

$\begin{array}{llllllllllllll}30 \ldots & 6.8 & .5 & 8.6 & 450 & 1.8 & <1 & <.1 & 65 & 67 & 10 & 20 & 1 & 15\end{array}$


Table 7. Carbon and major and trace elements in bed-sediment samples collected in Christmas, Bastrop, and Drum Bays, November 30, 1999-Continued

\begin{tabular}{|c|c|c|c|c|c|c|c|c|c|c|c|c|c|}
\hline & & & & & BASTH & ROP BAY & ONTINUED & & & & & & \\
\hline & GOLD & HOLMIUM & IRON & $\begin{array}{l}\text { LANTHA- } \\
\text { NUM }\end{array}$ & LEAD & LITHIUM & $\begin{array}{l}\text { MANGA- } \\
\text { NESE }\end{array}$ & MERCURY & $\begin{array}{l}\text { MOLYB- } \\
\text { DENUM }\end{array}$ & $\begin{array}{l}\text { NEODYM- } \\
\text { IUM }\end{array}$ & NICKEL & NIOBIUM & $\begin{array}{l}\text { SCAN- } \\
\text { DIUM }\end{array}$ \\
\hline DATE & $\begin{array}{c}\text { BOT MAT } \\
\text { FIELD } \\
\text { (UG/G) }\end{array}$ & $\begin{array}{c}\text { BOT MAT } \\
\text { FIELD } \\
\text { (UG/G) }\end{array}$ & $\begin{array}{l}\text { BOT MAT } \\
\text { FIELD } \\
\text { PERCENT }\end{array}$ & $\begin{array}{l}\text { BOT MAT } \\
\text { FIELD } \\
\text { (UG/G) }\end{array}$ & $\begin{array}{c}\text { BOT MAT } \\
\text { FIELD } \\
\text { (UG/G) }\end{array}$ & $\begin{array}{c}\text { BOT MAT } \\
\text { FIELD } \\
\text { (UG/G) }\end{array}$ & $\begin{array}{c}\text { BOT MAT } \\
\text { FIELD } \\
\text { (UG/G) }\end{array}$ & $\begin{array}{l}\text { BOT MAT } \\
\text { FIELD } \\
\text { (UG/G) }\end{array}$ & $\begin{array}{l}\text { BOT MAT } \\
\text { FIELD } \\
\text { (UG/G) }\end{array}$ & $\begin{array}{l}\text { BOT MAT } \\
\text { FIELD } \\
\text { (UG/G) }\end{array}$ & $\begin{array}{c}\text { BOT MAT } \\
\text { FIELD } \\
\text { (UG/G) }\end{array}$ & $\begin{array}{c}\text { BOT MAT } \\
\text { FIELD } \\
\text { (UG/G) }\end{array}$ & $\begin{array}{c}\text { BOT MAT } \\
\text { FIELD } \\
\text { (UG/G) }\end{array}$ \\
\hline IOV & & & & & & & & & & & & & \\
\hline $30 \ldots$ & $<1$ & $<1$ & 3.0 & 38 & 21 & 44 & 590 & .03 & .5 & 29 & 23 & 12 & 11 \\
\hline & $\begin{array}{l}\text { SELE- } \\
\text { NIUM }\end{array}$ & SILVER & $\begin{array}{l}\text { STRON- } \\
\text { TIUM }\end{array}$ & $\begin{array}{l}\text { TANTA- } \\
\text { LUM }\end{array}$ & $\begin{array}{l}\text { THAL- } \\
\text { LIUM } \\
\text { BED MAT }\end{array}$ & TIN & $\begin{array}{c}\text { TITA- } \\
\text { NIUM, } \\
\text { SED， BM }\end{array}$ & $\begin{array}{l}\text { VANA- } \\
\text { DIUM }\end{array}$ & $\begin{array}{l}\text { YTTER- } \\
\text { BIUM }\end{array}$ & YTTRIUM & ZINC & THORIUM & URANIUM \\
\hline & ВОТ МАТ & ВОT MAT & ВОТ MAT & ВОТ MAT & D SIEVE & ВОТ МАТ & WS , <63U & ВОТ МАТ & ВОТ МАТ & ВОТ МAT & BOT MAT & ВОТ MAT & BOT MAT \\
\hline DATE & $\begin{array}{l}\text { FIELD } \\
\text { (UG/G) }\end{array}$ & $\begin{array}{l}\text { FIELD } \\
\text { (UG/G) }\end{array}$ & $\begin{array}{l}\text { FIELD } \\
\text { (UG/G) }\end{array}$ & $\begin{array}{l}\text { FIELD } \\
\text { (UG/G) }\end{array}$ & $\begin{array}{l}\text { TOTAL } \\
\text { (UG/G) }\end{array}$ & $\begin{array}{l}\text { FIELD } \\
\text { (UG/G) }\end{array}$ & $\begin{array}{c}\text { REC } \\
\text { PERCENT }\end{array}$ & $\begin{array}{l}\text { FIELD } \\
\text { (UG/G) }\end{array}$ & $\begin{array}{l}\text { FIELD } \\
\text { (UG/G) }\end{array}$ & $\begin{array}{l}\text { FIELD } \\
\text { (UG/G) }\end{array}$ & $\begin{array}{l}\text { FIELD } \\
\text { (UG/G) }\end{array}$ & $\begin{array}{l}\text { FIELD } \\
\text { (UG/G) }\end{array}$ & $\begin{array}{l}\text { FIELD } \\
\text { (UG/G) }\end{array}$ \\
\hline & & & & & & & & & & & & & \\
\hline 30. & .2 & .4 & 120 & 1 & $<1$ & 3 & .340 & 93 & 2 & 22 & 86 & 11 & 2.4 \\
\hline
\end{tabular}

\begin{tabular}{|c|c|c|c|c|c|c|c|c|c|c|c|c|c|}
\hline & & & & & & DRUM $B$ & & & & & & & \\
\hline DATE & STATION & NUMBER & DATE & TIME & $\begin{array}{c}\text { CALCIUM } \\
\text { BOT MAT } \\
\text { FIELD } \\
\text { PERCENT }\end{array}$ & $\begin{array}{l}\text { MAGNE- } \\
\text { SIUM } \\
\text { BOT MAT } \\
\text { FIELD } \\
\text { PERCENT }\end{array}$ & $\begin{array}{l}\text { POTAS- } \\
\text { SIUM } \\
\text { BOT MAT } \\
\text { FIELD } \\
\text { PERCENT }\end{array}$ & $\begin{array}{l}\text { SODIUM } \\
\text { BOT MAT } \\
\text { FIELD } \\
\text { PERCENT }\end{array}$ & $\begin{array}{l}\text { SULFUR } \\
\text { BOT MAT } \\
\text { FIELD } \\
\text { PERCENT }\end{array}$ & $\begin{array}{c}\text { PHOS- } \\
\text { PHORUS } \\
\text { BOT MAT } \\
\text { FIELD } \\
\text { PERCENT }\end{array}$ & $\begin{array}{c}\text { CARBON, } \\
\text { INORG, } \\
\text { SED, BM } \\
\text { WS, <63U } \\
\text { (PER- } \\
\text { CENT) }\end{array}$ & $\begin{array}{c}\text { CARBON, } \\
\text { ORG + } \\
\text { INORG, } \\
\text { SED, BM } \\
\text { DW, REC } \\
\text { PERCENT }\end{array}$ & $\begin{array}{c}\text { CARBON, } \\
\text { ORGANIC } \\
\text { SED, BM } \\
\text { WS, <63U } \\
\text { (PER- } \\
\text { CENT) }\end{array}$ \\
\hline NOV & & & & & & & & & & & & & \\
\hline $30 \ldots$ & 29003509 & 5133801 & 19991130 & 0921 & 1.8 & 1.7 & 2.2 & 2.8 & .36 & .058 & .46 & 1.3 & .82 \\
\hline $30 \ldots$ & 29003509 & 5133801 & 19991130 & 0945 & 1.8 & 1.6 & 2.2 & 2.7 & .32 & .057 & .47 & 1.3 & .83 \\
\hline & $\begin{array}{l}\text { ALUM- } \\
\text { INUM }\end{array}$ & $\begin{array}{l}\text { ANTI- } \\
\text { MONY }\end{array}$ & ARSENIC & ARIUM & $\begin{array}{l}\text { BERYL- } \\
\text { LIUM }\end{array}$ & BISMUTH & CADMIUM & CERIUM & $\begin{array}{l}\text { CHRO- } \\
\text { MIUM }\end{array}$ & COBALT & COPPER & $\begin{array}{l}\text { EURO- } \\
\text { PIUM }\end{array}$ & GALLIUM \\
\hline & ВОТ МАТ & ВОT МАТ & ВОT MAT & BOT MAT & ВОТ МAT & BOT MAT & ВОT MAT & ВОT MAT & ВОТ МAT & ВОT MAT & ВОТ MAT & ВОТ МАТ & BOT MAT \\
\hline DATE & $\begin{array}{c}\text { FIELD } \\
\text { PERCENT }\end{array}$ & $\begin{array}{l}\text { FIELD } \\
\text { (UG/G) }\end{array}$ & $\begin{array}{l}\text { FIELD } \\
\text { (UG/G) }\end{array}$ & $\begin{array}{l}\text { FIELD } \\
\text { (UG/G) }\end{array}$ & $\begin{array}{l}\text { FIELD } \\
\text { (UG/G) }\end{array}$ & $\begin{array}{l}\text { FIELD } \\
\text { (UG/G) }\end{array}$ & $\begin{array}{l}\text { FIELD } \\
\text { (UG/G) }\end{array}$ & $\begin{array}{l}\text { FIELD } \\
\text { (UG/G) }\end{array}$ & $\begin{array}{l}\text { FIELD } \\
\text { (UG/G) }\end{array}$ & $\begin{array}{l}\text { FIELD } \\
\text { (UG/G) }\end{array}$ & $\begin{array}{l}\text { FIELD } \\
\text { (UG/G) }\end{array}$ & $\begin{array}{l}\text { FIELD } \\
\text { (UG/G) }\end{array}$ & $\begin{array}{l}\text { FIELD } \\
\text { (UG/G) }\end{array}$ \\
\hline NOV & & & & & & & & & & & & & \\
\hline $30 \ldots$ & 7.2 & .6 & 9.8 & 400 & 2.0 & $<1$ & $<.1$ & 68 & 71 & 11 & 23 & 1 & 16 \\
\hline $30 \ldots$ & 7.1 & .6 & 9.3 & 390 & 2.0 & $<1$ & $<.1$ & 64 & 69 & 11 & 22 & 1 & 16 \\
\hline & GOLD & HOLMIUM & IRON & $\begin{array}{l}\text { LANTHA- } \\
\text { NUM }\end{array}$ & LEAD & LITHIUM & $\begin{array}{l}\text { MANGA- } \\
\text { NESE }\end{array}$ & MERCURY & $\begin{array}{l}\text { MOLYB- } \\
\text { DENUM }\end{array}$ & $\begin{array}{l}\text { NEODYM- } \\
\text { IUM }\end{array}$ & NICKEL & NIOBIUM & $\begin{array}{l}\text { SCAN- } \\
\text { DIUM }\end{array}$ \\
\hline & ВОT MAT & ВОT MAT & ВОТ МАТ & ВOT MAT & ВОT MAT & ВОT MAT & ВОТ МАT & ВОТ MAT & ВОТ МAT & ВОТ MAT & BOT MAT & ВOT MAT & BOT MAT \\
\hline DATE & $\begin{array}{l}\text { FIELD } \\
\text { (UG/G) }\end{array}$ & $\begin{array}{l}\text { FIELD } \\
\text { (UG/G) }\end{array}$ & $\begin{array}{c}\text { FIELD } \\
\text { PERCENT }\end{array}$ & $\begin{array}{l}\text { FIELD } \\
\text { (UG/G) }\end{array}$ & $\begin{array}{l}\text { FIELD } \\
\text { (UG/G) }\end{array}$ & $\begin{array}{l}\text { FIELD } \\
\text { (UG/G) }\end{array}$ & $\begin{array}{l}\text { FIELD } \\
\text { (UG/G) }\end{array}$ & $\begin{array}{l}\text { FIELD } \\
\text { (UG/G) }\end{array}$ & $\begin{array}{l}\text { FIELD } \\
\text { (UG/G) }\end{array}$ & $\begin{array}{l}\text { FIELD } \\
\text { (UG/G) }\end{array}$ & $\begin{array}{l}\text { FIELD } \\
\text { (UG/G) }\end{array}$ & $\begin{array}{l}\text { FIELD } \\
\text { (UG/G) }\end{array}$ & $\begin{array}{l}\text { FIELD } \\
\text { (UG/G) }\end{array}$ \\
\hline NOV & & & & & & & & & & & & & \\
\hline $30 \ldots$ & $<1$ & 1 & 3.3 & 40 & 22 & 49 & 750 & .03 & .5 & 30 & 26 & 12 & 12 \\
\hline $30 \ldots$ & $<1$ & $<1$ & 3.3 & 38 & 21 & 48 & 740 & .03 & .5 & 29 & 25 & 12 & 12 \\
\hline & $\begin{array}{l}\text { SELE- } \\
\text { NIUM }\end{array}$ & SILVER & $\begin{array}{l}\text { STRON- } \\
\text { TIUM }\end{array}$ & $\begin{array}{l}\text { TANTA- } \\
\text { LUM }\end{array}$ & $\begin{array}{l}\text { THAL- } \\
\text { LIUM } \\
\text { BED MAT }\end{array}$ & TIN & $\begin{array}{l}\text { TITA- } \\
\text { NIUM, } \\
\text { SED, BM }\end{array}$ & $\begin{array}{l}\text { VANA- } \\
\text { DIUM }\end{array}$ & $\begin{array}{l}\text { YTTER- } \\
\text { BIUM }\end{array}$ & YTTRIUM & ZINC & THORIUM & URANIUM \\
\hline DATE & $\begin{array}{c}\text { BOT MAT } \\
\text { FIELD } \\
\text { (UG/G) }\end{array}$ & $\begin{array}{c}\text { BOT MAT } \\
\text { FIELD } \\
\text { (UG/G) }\end{array}$ & $\begin{array}{c}\text { BOT MAT } \\
\text { FIELD } \\
\text { (UG/G) }\end{array}$ & $\begin{array}{c}\text { BOT MAT } \\
\text { FIELD } \\
\text { (UG/G) }\end{array}$ & $\begin{array}{c}\text { D SIEVE } \\
\text { TOTAL } \\
\text { (UG/G) }\end{array}$ & $\begin{array}{c}\text { BOT MAT } \\
\text { FIELD } \\
\text { (UG/G) }\end{array}$ & $\begin{array}{c}\text { WS },<63 \mathrm{U} \\
\text { REC } \\
\text { PERCENT }\end{array}$ & $\begin{array}{c}\text { BOT MAT } \\
\text { FIELD } \\
\text { (UG/G) }\end{array}$ & $\begin{array}{l}\text { BOT MAT } \\
\text { FIELD } \\
\text { (UG/G) }\end{array}$ & $\begin{array}{l}\text { BOT MAT } \\
\text { FIELD } \\
\text { (UG/G) }\end{array}$ & $\begin{array}{c}\text { BOT MAT } \\
\text { FIELD } \\
\text { (UG/G) }\end{array}$ & $\begin{array}{c}\text { BOT MAT } \\
\text { FIELD } \\
\text { (UG/G) }\end{array}$ & $\begin{array}{c}\text { BOT MAT } \\
\text { FIELD } \\
\text { (UG/G) }\end{array}$ \\
\hline NOV & & & & & & & & & & & & & \\
\hline 30 & .2 & .4 & 130 & 1 & $<1$ & 3 & .360 & 98 & 2 & 24 & 91 & 12 & 2.5 \\
\hline 30. & .2 & 4 & 120 & 1 & $<1$ & 3 & .330 & 96 & 2 & 24 & 89 & 11 & 2.3 \\
\hline
\end{tabular}


Table 8. Semivolatile organic compounds (mostly polycyclic aromatic hydrocarbons) in bed-sediment samples collected in Christmas, Bastrop, and Drum Bays, November 30, 1999

$[\mu \mathrm{g} / \mathrm{kg}$, micrograms per kilogram; <, less than; NA, not analyzed]

\begin{tabular}{|c|c|c|c|c|c|c|}
\hline \multirow{2}{*}{ Compound } & \multicolumn{5}{|c|}{$\begin{array}{c}\text { Concentration } \\
(\mu \mathbf{g} / \mathbf{k g})\end{array}$} & \multirow{2}{*}{$\begin{array}{c}\begin{array}{c}\text { Recovery } \\
\text { (percent) }\end{array} \\
\text { Laboratory } \\
\text { matrix spike }\end{array}$} \\
\hline & $\begin{array}{l}\text { Christmas } \\
\text { Bay sample }\end{array}$ & $\begin{array}{c}\text { Bastrop Bay } \\
\text { sample }\end{array}$ & $\begin{array}{l}\text { Drum Bay } \\
\text { sample }\end{array}$ & $\begin{array}{c}\text { Drum Bay } \\
\text { split sample }\end{array}$ & $\begin{array}{l}\text { Laboratory } \\
\text { blank }\end{array}$ & \\
\hline Phenol & ${ }^{1} 17$ & ${ }^{1} 14.3$ & ${ }^{1} 14.9$ & ${ }^{1} 11.3$ & 9.9 & 65.92 \\
\hline$p$-Cresol & ${ }^{2} 4.8$ & ${ }^{2} 3.7$ & ${ }^{2} 5.3$ & ${ }^{2} 4.9$ & $<5$ & ${ }^{2} 14.52$ \\
\hline C8-Alkylphenol & NA & NA & NA & NA & NA & NA \\
\hline Naphthalene & $<10$ & $<10$ & ${ }^{2} 1.1$ & ${ }^{2} 1.2$ & $<5$ & 71.74 \\
\hline $\mathrm{C} 1-128$ isomers & $<10$ & ${ }^{2} 2.6$ & ${ }^{2} 3.7$ & 24.2 & $<5$ & NA \\
\hline 2-Ethylnaphthalene & ${ }^{2} 1.6$ & ${ }^{2} 1.9$ & ${ }^{2} 2.4$ & ${ }^{2} 2.4$ & $<5$ & 70.08 \\
\hline 2,6-Dimethylnaphthalene & ${ }^{2} 2.6$ & ${ }^{2} 5.0$ & ${ }^{2} 6.2$ & 5.5 & $<5$ & 66.89 \\
\hline 1,6-Dimethylnaphthalene & ${ }^{2} 1.6$ & ${ }^{2} 2.5$ & ${ }^{2} 3.4$ & ${ }^{2} 3.1$ & $<5$ & 67.12 \\
\hline $\mathrm{C} 2-128$ isomers & ${ }^{2} 5.4$ & 11.5 & 11.7 & 13.1 & $<5$ & NA \\
\hline Acenaphthylene & ${ }^{2} 1.1$ & ${ }^{2} 2.0$ & ${ }^{2} 2.4$ & ${ }^{2} 3.2$ & $<5$ & 67.75 \\
\hline 1,2-Dimethylnaphthalene & ${ }^{2} 1.9$ & ${ }^{2} 1.6$ & ${ }^{2} 2.4$ & ${ }^{2} 2.0$ & $<5$ & 69.50 \\
\hline Acenaphthene & $<10$ & $<10$ & $<10$ & $<10$ & $<5$ & 70.05 \\
\hline $\mathrm{C} 3-128$ isomers & ${ }^{2} 3.9$ & ${ }^{2} 8.1$ & 10.2 & 11.1 & $<5$ & NA \\
\hline 2,3,6-Trimethylnaphthalene & ${ }^{2} 1.4$ & $<10$ & ${ }^{2} 2.9$ & $<10$ & $<5$ & 77.37 \\
\hline $9 H$-Fluorene & $<10$ & ${ }^{2} 1.4$ & ${ }^{2} 1.9$ & ${ }^{2} 1.9$ & $<5$ & 71.03 \\
\hline $\mathrm{C} 4-128$ isomers & $<10$ & $<10$ & $<10$ & $<10$ & $<5$ & NA \\
\hline 1-Methyl-9H-fluorene & $<10$ & ${ }^{2} 1.4$ & ${ }^{2} 1.7$ & $<10$ & $<5$ & 74.15 \\
\hline $\mathrm{C} 1-166$ isomers & NA & NA & NA & NA & NA & NA \\
\hline Dibenzothiophene & NA & NA & NA & NA & NA & NA \\
\hline Phenanthrene & ${ }^{2} 2.1$ & ${ }^{2} 3.2$ & ${ }^{2} 5.9$ & 24.6 & $<5$ & 75.14 \\
\hline Anthracene & ${ }^{2} 1.7$ & ${ }^{2} 3.0$ & ${ }^{2} 3.6$ & ${ }^{2} 3.9$ & $<5$ & 74.11 \\
\hline Acridine & NA & NA & NA & NA & NA & NA \\
\hline Phenanthridine & NA & NA & NA & NA & NA & NA \\
\hline $9 H$-Carbazol & NA & NA & NA & NA & NA & NA \\
\hline C5-128 isomers & $<10$ & $<10$ & $<10$ & $<10$ & $<5$ & NA \\
\hline $\mathrm{C} 2-166$ isomers & NA & NA & NA & NA & NA & NA \\
\hline 2-Methylanthracene & ${ }^{2} 1.1$ & ${ }^{2} 1.6$ & ${ }^{2} 2.2$ & ${ }^{2} 2.2$ & $<5$ & 70.43 \\
\hline 4,5-Methylenephenanthrene & $<10$ & ${ }^{2} 1.5$ & ${ }^{2} 2.0$ & ${ }^{2} 2.1$ & $<5$ & 77.94 \\
\hline $\mathrm{C} 1-178$ isomers & ${ }^{2} 3.2$ & ${ }^{2} 4.8$ & ${ }^{2} 6.6$ & ${ }^{2} 7.1$ & $<5$ & NA \\
\hline 1-Methylphenanthrene & $<10$ & ${ }^{2} 1.1$ & ${ }^{2} 1.4$ & ${ }^{2} 1.5$ & $<5$ & 75.48 \\
\hline $\mathrm{C} 3-166$ isomers & NA & NA & NA & NA & NA & NA \\
\hline $\mathrm{C} 2-178$ isomers & $<10$ & ${ }^{2} 5.2$ & ${ }^{2} 4.4$ & ${ }^{2} 6.6$ & $<5$ & NA \\
\hline
\end{tabular}

Footnotes at end of table. 
Table 8. Semivolatile organic compounds (mostly polycyclic aromatic hydrocarbons) in bed-sediment samples collected in Christmas, Bastrop, and Drum Bays, November 30, 1999-Continued

\begin{tabular}{|c|c|c|c|c|c|c|}
\hline \multirow{2}{*}{ Compound } & \multicolumn{5}{|c|}{$\begin{array}{c}\text { Concentration } \\
(\mu \mathrm{g} / \mathrm{kg})\end{array}$} & \multirow{2}{*}{$\begin{array}{c}\begin{array}{c}\text { Recovery } \\
\text { (percent) }\end{array} \\
\text { Laboratory } \\
\text { matrix spike }\end{array}$} \\
\hline & $\begin{array}{l}\text { Christmas } \\
\text { Bay sample }\end{array}$ & $\begin{array}{c}\text { Bastrop Bay } \\
\text { sample }\end{array}$ & $\begin{array}{l}\text { Drum Bay } \\
\text { sample }\end{array}$ & $\begin{array}{c}\text { Drum Bay } \\
\text { split sample }\end{array}$ & $\begin{array}{l}\text { Laboratory } \\
\text { blank }\end{array}$ & \\
\hline Fluoranthene & ${ }^{2} 4.0$ & ${ }^{27} 7.6$ & ${ }^{2} 9.4$ & 10.3 & $<5$ & 89.25 \\
\hline Pyrene & 24.3 & ${ }^{2} 7.2$ & 10.9 & 11.7 & $<5$ & 90.64 \\
\hline $\mathrm{C} 3-178$ isomers & $<10$ & $<10$ & 24.1 & ${ }^{2} 5.1$ & $<5$ & NA \\
\hline $\mathrm{C} 4-178$ isomers & $<10$ & $<10$ & $<10$ & $<10$ & $<5$ & NA \\
\hline 1-Methylpyrene & $<10$ & ${ }^{2} 1.6$ & ${ }^{2} 2.3$ & ${ }^{2} 2.2$ & $<5$ & 91.65 \\
\hline $\mathrm{C} 1-202$ isomers & ${ }^{2} 3.6$ & ${ }^{2} 7.1$ & ${ }^{2} 8.3$ & ${ }^{2} 2.4$ & $<5$ & NA \\
\hline $\mathrm{C} 2-202$ isomers & 24.1 & ${ }^{2} 7.5$ & 27.2 & ${ }^{2} 1.7$ & $<5$ & NA \\
\hline C5-178 isomers & $<10$ & $<10$ & $<10$ & $<10$ & $<5$ & NA \\
\hline $\operatorname{Benz}(a)$ anthracene & ${ }^{2} 2.4$ & ${ }^{2} 4.6$ & ${ }^{2} 5.7$ & ${ }^{2} 6.1$ & $<5$ & 93.72 \\
\hline Chrysene & ${ }^{2} 1.3$ & ${ }^{2} 3.3$ & ${ }^{2} 3.2$ & 23.9 & $<5$ & 80.95 \\
\hline C3-202 isomers & $<10$ & $<10$ & $<10$ & ${ }^{2} 1.7$ & $<5$ & NA \\
\hline $\mathrm{C} 1-228$ isomers & ${ }^{2} 2.0$ & 24.9 & 23.4 & 24.5 & $<5$ & NA \\
\hline $\mathrm{C} 4-202$ isomers & $<10$ & $<10$ & $<10$ & ${ }^{2} 7.5$ & $<5$ & NA \\
\hline C5-202 isomers & $<10$ & $<10$ & $<10$ & ${ }^{2} 2.0$ & $<5$ & NA \\
\hline $\mathrm{C} 2-228$ isomers & $<10$ & $<10$ & $<10$ & $<10$ & $<5$ & NA \\
\hline Benzo(b)fluoranthene & 24.8 & 10.3 & 10.6 & 12.3 & $<5$ & 93.51 \\
\hline $\operatorname{Benzo}(k)$ fluoranthene & ${ }^{2} 1.7$ & 23.8 & ${ }^{2} 5.8$ & ${ }^{2} 5.1$ & $<5$ & 76.43 \\
\hline $\operatorname{Benzo}(e)$ pyrene & ${ }^{2} 2.7$ & ${ }^{2} 5.8$ & ${ }^{2} 6.5$ & ${ }^{2} 7.0$ & $<5$ & 78.21 \\
\hline Benzo $(a)$ pyrene & ${ }^{2} 2.8$ & ${ }^{2} 6.3$ & ${ }^{2} 6.7$ & ${ }^{2} 7.3$ & $<5$ & 81.69 \\
\hline Perylene & ${ }^{2} 9.3$ & 14.2 & 13.8 & 14.8 & $<5$ & 67.31 \\
\hline $\mathrm{C} 1-252$ isomers & 24.2 & 10.7 & ${ }^{2} 9.6$ & 10.6 & $<5$ & NA \\
\hline C3-228 isomers & $<10$ & $<10$ & $<10$ & $<10$ & $<5$ & NA \\
\hline $\mathrm{C} 2-252$ isomers & $<10$ & $<10$ & ${ }^{2} 5.2$ & $<10$ & $<5$ & NA \\
\hline $\mathrm{C} 4-228$ isomers & $<10$ & $<10$ & $<10$ & $<10$ & $<5$ & NA \\
\hline Indeno(1,2,3-cd)pyrene & ${ }^{2} 3.3$ & ${ }^{2} 5.6$ & ${ }^{2} 7.9$ & ${ }^{2} 8.0$ & $<5$ & 85.64 \\
\hline $\operatorname{Dibenz}(a, h)$ anthracene & ${ }^{2} 1.4$ & ${ }^{2} 2.4$ & ${ }^{2} 3.1$ & ${ }^{2} 2.7$ & $<5$ & 76.55 \\
\hline Benzo(ghi)perylene & ${ }^{2} 2.8$ & ${ }^{2} 4.5$ & ${ }^{2} 5.8$ & ${ }^{2} 6.2$ & $<5$ & 65.67 \\
\hline $\mathrm{C} 3-252$ isomers & $<10$ & $<10$ & $<10$ & $<10$ & $<5$ & NA \\
\hline $\mathrm{C} 4-252$ isomers & $<10$ & $<10$ & $<10$ & $<10$ & $<5$ & NA \\
\hline C5-228 isomers & $<10$ & $<10$ & ${ }^{2} 1.7$ & $<10$ & $<5$ & NA \\
\hline C5-252 isomers & $<10$ & $<10$ & $<5$ & $<10$ & $<5$ & NA \\
\hline Coronene & ${ }^{2} 1.1$ & ${ }^{2} 1.6$ & ${ }^{2} 2.6$ & ${ }^{2} 2.4$ & $<5$ & ${ }^{2} 29.32$ \\
\hline
\end{tabular}

${ }^{1}$ Contaminated.

2 Estimated. 
Table 9. Organochlorine pesticides in bed-sediment samples collected in Christmas, Bastrop, and Drum Bays, November 30, 1999

[Samples collected from top 3 centimeters of bottom sediments. All samples based on dry weight; units are micrograms per kilogram; <, less than]

\begin{tabular}{|c|c|c|c|c|c|c|c|c|c|c|}
\hline Station name & $\begin{array}{c}\text { Gamma-HCH } \\
\text { (lindane) }\end{array}$ & & Heptachlor & Aldrin & $\begin{array}{c}\text { Heptachlor } \\
\text { epoxide }\end{array}$ & Chlordane & Endosulfan I & Dieldrin & $\begin{array}{l}p, p^{\prime}- \\
\text { DDE }\end{array}$ & Endrin \\
\hline Christmas Bay & $<0.5$ & & $<0.5$ & $<0.5$ & $<0.5$ & $<0.5$ & $<0.5$ & $<0.5$ & $<0.5$ & $<0.5$ \\
\hline Bastrop Bay & $<.5$ & & $<.5$ & $<.5$ & $<.5$ & $<.5$ & $<.5$ & $<.5$ & $<.5$ & $<.5$ \\
\hline Drum Bay & $<1.0$ & & $<1.0$ & $<1.0$ & $<1.0$ & $<1.0$ & $<1.0$ & $<1.0$ & $<1.0$ & $<1.0$ \\
\hline Drum Bay split & $<1.25$ & & $<1.25$ & $<1.25$ & $<1.25$ & $<1.25$ & $<1.25$ & $<1.25$ & $<1.25$ & $<1.25$ \\
\hline Station name & $\begin{array}{l}p, p^{\prime}- \\
\text { DDD }\end{array}$ & $\begin{array}{l}p, p:- \\
\text { DDT }\end{array}$ & Metl & $\begin{array}{l}p, p^{\prime}- \\
\text { hoxychlor }\end{array}$ & Mirex & Toxaphene & $\begin{array}{c}\text { РСB } \\
\text { Aroclor } 1242\end{array}$ & $\begin{array}{c}\text { РСB } \\
\text { Aroclor } 12\end{array}$ & $254 \mathrm{Ar}$ & $\begin{array}{c}\text { РСB } \\
\text { Aroclor } 1260\end{array}$ \\
\hline Christmas Bay & $<0.5$ & $<0.5$ & & $<0.5$ & $<0.5$ & $<50$ & $<5.0$ & $<5.0$ & & $<5.0$ \\
\hline Bastrop Bay & $<.5$ & $<.5$ & & $<.5$ & $<.5$ & $<50$ & $<5.0$ & $<5.0$ & & $<5.0$ \\
\hline Drum Bay & $<1.0$ & $<1.0$ & & $<4.0$ & $<1.0$ & $<50$ & $<5.0$ & $<5.0$ & & $<5.0$ \\
\hline Drum Bay split & $<1.25$ & $<1.25$ & & $<5.0$ & $<1.25$ & $<125$ & $<5.0$ & $<5.0$ & & $<5.0$ \\
\hline
\end{tabular}


District Chief

U.S. Geological Survey

8027 Exchange Dr.

Austin, TX 78754-4733 